\title{
CANINE DISTEMPER
}

ITS COMPLICATIONS, SEQUELAE

AND TREATMENT

HAMILTON KIRK 


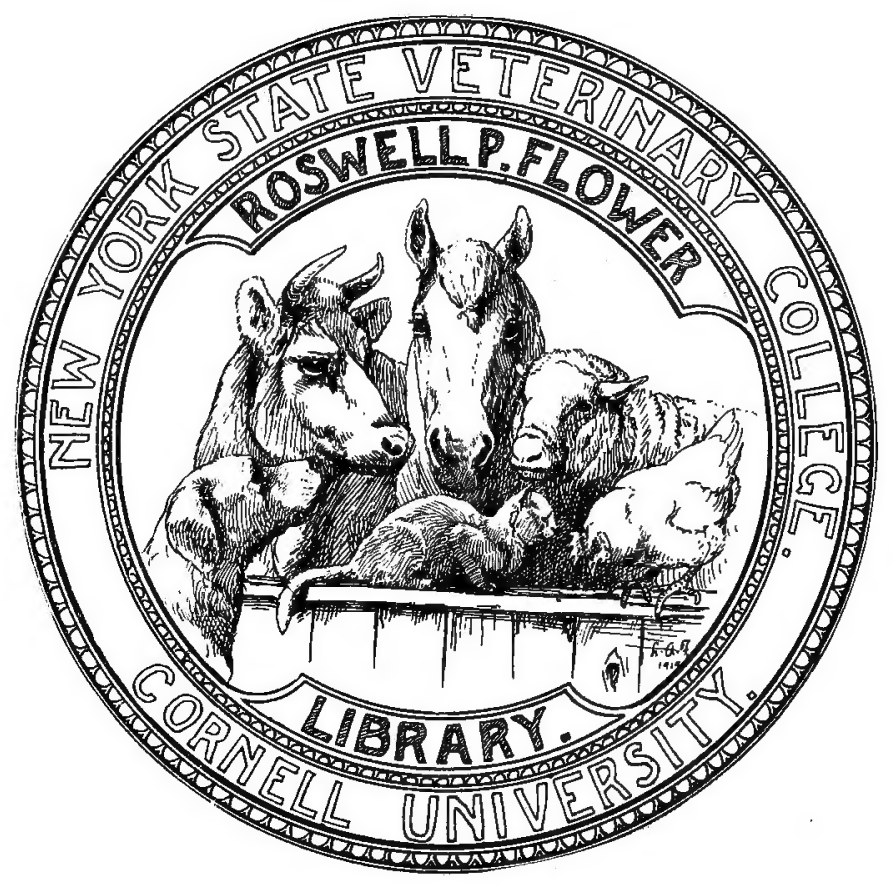




$$
C_{N} 4 / 6
$$

SF 991.K59 Cornell University Library

Canine distemper, its complications, sequ

3 1924000943617

Date Due

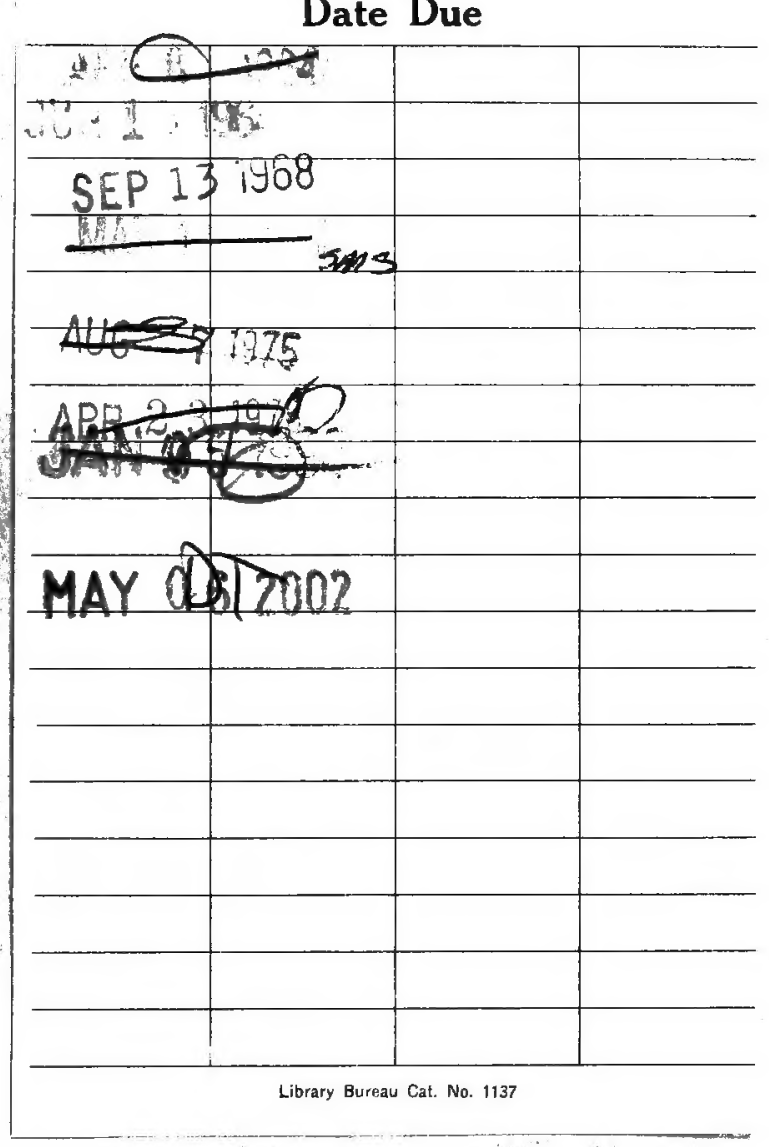




\section{Cornell University Library}

The original of this book is in the Cornell University Library.

There are no known copyright restrictions in the United States on the use of the text. 
CANINE DISTEMPER 








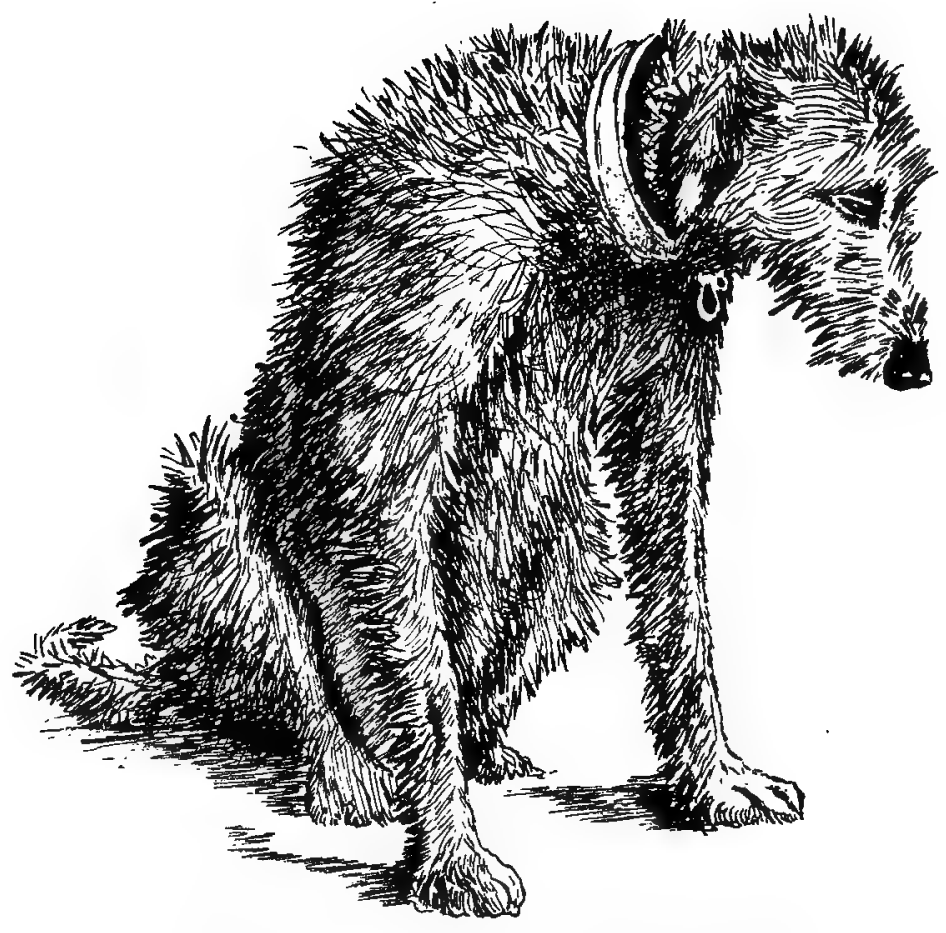

Characteristic Attitude of a Sick Dog 


\title{
CANINE DISTEMPER
}

ITS COMPLICATIONS, SEQUELÆ, AND TREATMENT

\author{
BY \\ HAMILTON KIRK \\ MEMBER OF THE ROYAL COLLEGE OF VETERINARY SURGEONS \\ FELLOW OF THE ROVAL SOCIETY OF MEDICINE \\ CAPTAIN, ROYAL ARMY VETERINARY CORPS (T.F.)
}

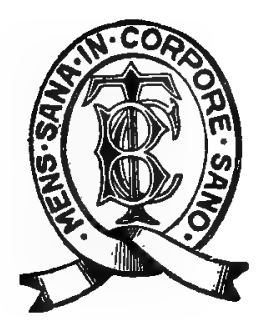

LONDON

\section{BAILLIERE, TINDALL AND COX}

8, HENRIETTA STREET, COVENT GARDEN

1922

[All rights reserved] 
Flower Likny.

No.6476

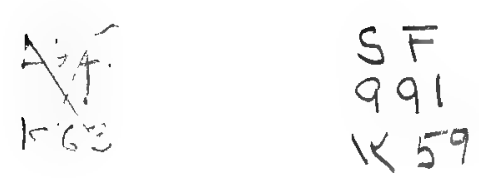


TO

FREDERICK T. G. HOBDAY,

C.M.G., F.R.C.V.S., F.R.S.E.,

AS A SLIGHT TOKEN OF ESTEEM AND APPRECIATION OF HIS

VALUABLE LITERARY CONTRIBUTIONS TO VETERINARY

SCIENCE, AND IN SINCERE GRATITUDE FOR HIS

UNCEASING EFFORTS TO ENHANCE THE

INTERESTS OF THE VETERINARY

PROFESSION,

THIS WORK IS DEDICATED BY THE AUTHOR 



\section{PREFACE}

IN presenting to the public this, the only work dealing exclusively with canine distemper, its complications and sequelæ; I must at the outset express regret at my inability to further elucidate the mysteries-chiefly pertaining to etiology - which continue to exercise our minds and demand untiring investigations. This question of etiology has baffled the highest authorities since the disease was first recognised, and whilst it has unhappily not fallen to my humble lot to solve the problem, I have had to be content to review the malady generally and recapitulate the carefully collected theories and clinical facts devolved from the observations and investigations of eminent veterinary and other authorities during the last seventy years.

I venture to hope that this work will fulfil a distinct and apparently long felt want; inasmuch as, formerly, those desiring detailed information upon any phase of the disease were obliged to search endless papers, works, and proceedings of societies, before any tangible and collective facts could be elicited.

I have endeavoured to present in readable form every detail concerning the spread of distemper, how it might be avoided, its symptoms, treatment, and sequelæ, not omitting the latest ascertainable bacteriological findings relative to its etiology and preventive inoculation, that the whole may prove of service as a book of reference for the practitioner and student.

Distemper has probably not received the attention it would have done had the disease been communicable to man, or the cause of a very heavy annual pecuniary loss 
to the country. Although the dog may be considered of minor importance in our economic life, yet he is very dear to us, and there is probably none but wishes his welfare and immunity to suffering.

If in any measure I shall have contributed to this end by placing within the confines of one volume such knowledge as exists to date in reach of the dog-loving population, I shall feel amply rewarded for my efforts. In any case I trust I have discredited some of the popular fallacies relating to distemper in dogs, and that the reader, no less than his canine friend, will profit by what is written.

My grateful thanks are due to Professor Hobday, Dr. Ferry, and Dr. Copeman for the generous way in which they placed at my disposal drawings, literature, and the fruits of their ripe experience, and particularly, in the case of the first-named gentleman, for kindly. undertaking the onerous task of reading through the proofs. I am also indebted to Messrs. Parke, Davis and Co., who have kindly given me access to much literature dealing with the malady; to Messrs. Müller and Glass for the permission accorded me for the use of several of the illustrations herein depicted, and to Messrs. Arnold and Sons for those of instruments. The frontispiece has been redrawn from a copy supplied by kind permission of The Bazaar, Exchange and Mart.

H. K,

67, WigMore STREET,

LONDON, W. I.

April, I922. 


\section{CONTENTS}

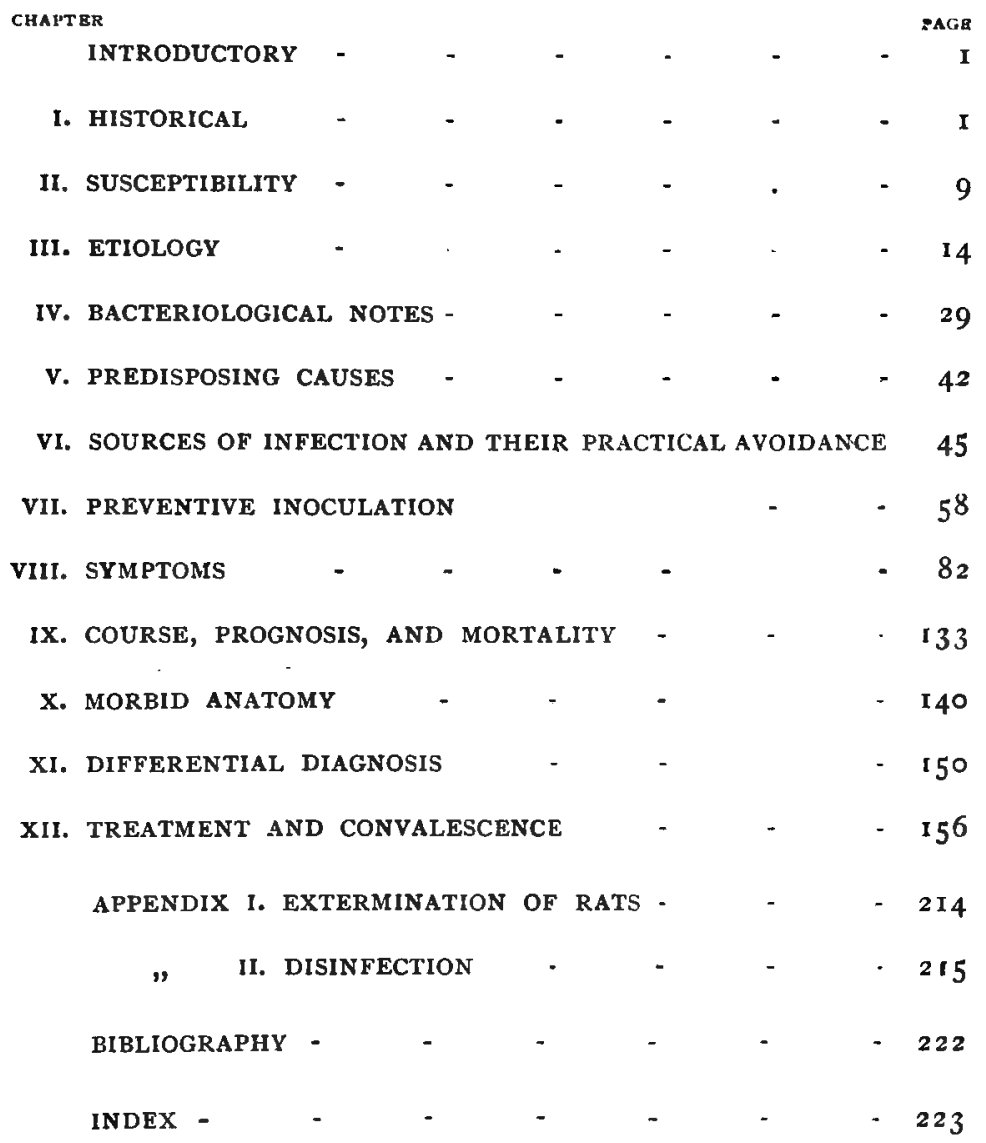




\section{LIST OF ILLUSTRATIONS}

FIG.

CHARACTERISTIC ATTITUDE OF A SICK DOG Frontispiece

I. BACILLUS BRONCHISEPTICUS - $\quad$ - $\quad$ - $\quad$ - I5

2. DR. COPEMAN'S BACILLUS - - - $\quad$ - $\quad$ - 25

3. PASTEURELla CANIS OF LIGNiERES AND PHiSAlix - 33

4. BACILLI ASSOCIATED WITH DISTEMPER - - $\quad$ - 37

5. MICROCOCCI ASSOCIATED WITH DISTEMPER - 39

6. FIELD OF PERCUSSION AND LOCATION OF HEART-BEAT - 89

7. LEUCOMA OF THE LEFT EYE - $\quad$ - $\quad$ - $\quad 96$

8. GREY CATARACT OF BOTH EYES- - - - - 97

9. PROLAPSE OF THE RECTUM - $\quad$ - $\quad$ - $\quad$ - 102

IO. PARALYSIS OF THE CORD - $\quad$ - $\quad$ - $\quad$ - II4

II. PARALYSIS OF THE POSTERIOR EXTREMITIES - - II5

I2. TEMPERATURE CHART OF PROTRACTED FATAL CASE - I2I

i 2a. TEMPERATURE CHART OF BENIGN CASE - - $\quad$ - 122

I3. DOG WITH ASCITES - $\quad$ - $\quad$ - $\quad$ - $\quad$ - $\quad$ - 125

14. PASSING THE CATHETER - - $\quad$ - $\quad$ - 130

I5. MALE VESICAL CATHETER - - - - - - I $\mathbf{I}$

16. FEMALE VESICAL CATHETER - - - - - I 3 I

17. URINE OF A DOG WITH CYSTITIS - $-\quad$ - I3I

I8. COAT FOR CHEST PROTECTION - - - - I60

I9. INHALATION OF STEAM - - $\quad$ - $\quad$ - $\quad$ - I6I

20. RECTAL LAVAGE $\quad$ - $\quad$ - $\quad$ - $\quad$ - $\quad$ - I7I

2 I. SOFT, PLIABLE CATHETER FOR RECTAL USE - $\quad$ I 72

21a. RUBBER RECTAL TUBE FOR RECTAL LAVAGE - $\quad$ I 72

22. USEFUL CHART FOR INFIRMARY USE - - - $\quad$ I74

23. METHOD OF GIVING FLUID MEDICINE - - - $\quad$ - 184

24. METHOD OF GIVING A PILL - - - $\quad$ - $\quad$ - 192

25. ASPIRATOR FOR USE IN PARACENTESIS ABDOMINIS - 194

26. IRRIGATING THE BLADDER - - - - - $\quad$ - 205

27. CLEANING THE INTERIOR OF THE EAR - - 207

28. CAP FOR PROTECTING THE EARS - - $\quad$ - 209 


\title{
CANINE DISTEMPER
}

\section{ITS COMPLICATIONS AND SEQUELAE}

\author{
INTRODUCTORY \\ French : Maladie des Chiens. Maladie du jeune âge. \\ German : Hundestaupe. Hundekrankheit. \\ Spantish : Reuma. \\ Italian: Cimurro.
}

\section{DEFINITION}

Nine Distemper is a specific, malignant; contagious and infectious catarrhal fever, affecting all mucous membranes, but primarily those of the respiratory tract. It is chiefly, but not essentially, a disease of young animals of the canine and feline race, appearing sporadically, enzoötically, or epizoötically, and frequently fraught with very serious complications and sequelæ.

\section{CHAPTER I \\ HISTORICAL \\ SYNONYMS AND HISTORY}

IN consequence of the very diverse character of the symptoms often presented during an attack of distemper, the malady has been known variously as influenza, malignant catarrhal fever, canine plague, broncho-pneumonia, gastro-enteritis, canine typhus, canine small-pox, and several other names, all of which, however, have 
gradually fallen into disuse in favour of the universally popular term-Canine Distemper.

During the seventeenth and eighteenth centuries, the word "distemper" was indiscriminately used to signify almost any epidemic of man or epizoötic of beast, though the meaning of the term. has gradually narrowed down to indicate exclusively the specific disease now under consideration. Among the various writers, both English and foreign, there appears to exist a considerable conflict of opinion as to whether distemper originated first in this country or was imported into Great Britain from the Continent. According to one author, a serious epizoötic raged in France about 1740 , and we learn from others that this was followed, about ten years later, by an outbreak in Germany, and in 1760 by one in England; it then appeared to sweep northwards into Russia, Siberia, Norway, Sweden, and Greenland, assuming in the last-named country a most virulent form upon its first appearance in 1859.

In the works of some of the ancient observers and authors, we may find vague allusions to outbreaks of epizoötic diseases among dogs in various parts of the world as far back as the year A.D. 1028; but whether in the absence of any detailed description of the symptoms we could safely ascribe the outbreaks as due to distemper is very doubtful. On consulting Fleming's "History of Animal Plagues," the following interesting facts are elicited :

A.D.

"1028. In the present year an invasion of cicadæ and caterpillars in Bohemia following a very plentiful harvest. Innumerable swarms of butterflies appeared, so that everything green in garden and field or in woods was devoured. Dense and foulsmelling vapours had preceded this visitation, rising, as they did, about Easter. After these insects had 
A.D.

eaten everything up, they themselves increased the stench ; the trees also, stripped of their leaves, died and rotted. As a consequence, there was a great mortality amongst men and animals, but especially in dogs in the autumn. England and Gaul, and, indeed, the whole of Europe, suffered in the same way and from the same causes.

"1414. According to Saxo Grammaticus, a severe form of dysentery ravaged Germany, affecting horses, cattle, dogs, and cats, as well as man.

" 1603 . Very inclement season in London, and pestilence among mankind which was supposed to have been introduced from the Low Countries. A famine prevailed, and extensive disease among all animals, but particularly cattle. Even dogs suffered greatly.

"I690. Italy. "On March I3, I observed an epidemic among dogs at Anda of an anginous character. After a very misty night, domestic and sporting dogs, besides three others; all perished in the town, of which, out of curiosity, I took twenty-one for examination, and I found their necks swollen externally and their fauces internally, while the muscles of their throats were much inflamed. Wirth ascribes the losses among animals in Italy to anthrax.'

" I 714. In March of this year, canine distemper raged in all the southern provinces of France as an epizoöty complicated with gangrenous angina.

" I715. In Germany and France an epizoöty of gastrobronchitis raged amongst dogs.

" 1735. Alloa, who was a resident in South America until 1746 , is the first author who has told us of the existence of, and describes the distemper of, dogs of that country.

"I76r. In this year there began a great epizoöty amongst dogs, which appears to have been what is since vulgarly termed 'distemper.' Before this period it appears to have been very rare or almost unknown, and since its outbreak at that time, it has lingered among the canine species to this day. It seems to have been first noted in Spain. This year there was observed at Madrid a deadly epizoöty among 
gradually fallen into disuse in favour of the universally popular term-Canine Distemper.

During the seventeenth and eighteenth centuries, the word "distemper" was indiscriminately used to signify almost any epidemic of man or epizoötic of beast, though the meaning of the term. has gradually narrowed down to indicate exclusively the specific disease now under consideration. Among the various writers, both English and foreign, there appears to exist a considerable conflict of opinion as to whether distemper originated first in this country or was imported into Great Britain from the Continent. According to one author, a serious epizoötic raged in France about $\mathrm{I} 740$, and we learn from others that this was followed, about ten years later, by an outbreak in Germany, and in 1760 by one in England; it then appeared to sweep northwards into Russia, Siberia, Norway, Sweden, and Greenland, assuming in the last-named country a most virulent form upon its first appearance in 1859 .

In the works of some of the ancient observers and authors, we may find vague allusions to outbreaks of epizoötic diseases among dogs in various parts of the world as far back as the year A.D. 1028; but whether in the absence of any detailed description of the symptoms we could safely ascribe the outbreaks as due to distemper is very doubtful. On consulting Fleming's "History of Animal Plagues," the following interesting facts are elicited :

A.D.

"1028. In the present year an invasion of cicadæ and caterpillars in Bohemia following a very plentiful harvest. Innumerable swarms of butterflies appeared, so that everything green in garden and field or in woods was devoured. Dense and foulsmelling vapours had preceded this visitation, rising, as they did, about Easter. After these insects had 
A.D.

eaten everything up, they themselves increased the stench; the trees also, stripped of their leaves, died and rotted. As a consequence, there was a great mortality amongst men and animals, but especially in dogs in the autumn. England and Gaul, and, indeed, the whole of Europe, suffered in the same way and from the same causes.

"I4I4. According to Saxo Grammaticus, a severe form of dysentery ravaged Germany, affecting horses, cattle, dogs, and cats, as well as man.

" 1603 . Very inclement season in London, and pestilence among mankind which was supposed to have been introduced from the Low Countries. A famine prevailed, and extensive disease among all animals, but particularly cattle. Even dogs suffered greatly.

"I690. Italy. 'On March I3, I observed an epidemic among dogs at Anda of an anginous character. After a very misty night, domestic and sporting dogs, besides three others; all perished in the town, of which, out of curiosity, I took twenty-one for examination, and I found their necks swollen externally and their fauces internally, while the muscles of their throats were much inflamed. Wirth ascribes the losses among animals in Italy to anthrax.'

"I7I4. In March of this year, canine distemper raged in all the southern provinces of France as an epizoöty complicated with gangrenous angina.

"I7I5. In Germany and France an epizoöty of gastrobronchitis raged amongst dogs.

“ 1735. Alloa, who was a résident in South America until I746, is the first author who has told us of the existence of, and describes the distemper of, dogs of that country.

"1761. In this year there began a great epizoöty amongst dogs, which appears to have been what is since vulgarly termed 'distemper.' Before this period it appears to have been very rare or almost unknown, and since its outbreak at that time, it has lingered among the canine species to this day. It seems to have been first noted in Spain. This year there was observed at Madrid a deadly epizoöty among 
gradually fallen into disuse in favour of the universally popular term-Canine Distemper.

During the seventeenth and eighteenth centuries, the word "distemper" was indiscriminately used to signify almost any epidemic of man or epizoötic of beast, though the meaning of the term has gradually narrowed down to indicate exclusively the specific disease now under consideration. Among the various writers, both English and foreign, there appears to exist a considerable conflict of opinion as to whether distemper originated first in this country or was imported into Great Britain from the Continent. According to one author, a serious epizoötic raged in France about 1740, and we learn from others that this was followed, about ten years later, by an outbreak in Germany, and in I760 by one in England; it then appeared to sweep northwards into Russia, Siberia, Norway, Sweden, and Greenland, assuming in the last-named country a most virulent form upon its first appearance in 1859 .

In the works of some of the ancient observers and authors, we may find vague allusions to outbreaks of epizoőtic diseases among dogs in various parts of the world as far back as the year A.D. I028; but whether in the absence of any detailed description of the symptoms we could safely ascribe the outbreaks as due to distemper is very doubtful. On consulting Fleming's "History of Animal Plagues," the following interesting facts are elicited :

A.D.

"1028. In the present year an invasion of cicadæ and caterpillars in Bohemia following a very plentiful harvest. Innumerable swarms of butterflies appeared, so that everything green in garden and field or in woods was devoured. Dense and foulsmelling vapours had preceded this visitation, rising, as they did, about Easter. After these insects had 
A.D.

eaten everything up, they themselves increased the stench ; the trees also, stripped of their leaves, died and rotted. As a consequence, there was a great mortality amongst men and animals, but especially in dogs in the autumn. England and Gaul, and, indeed, the whole of Europe, suffered in the same way and from the same causes.

"I4I4. According to Saxo Grammaticus, a severe form of dysentery ravaged Germany, affecting horses, cattle, dogs, and cats, as well as man.

" 1603 . Very inclement season in London, and pestilence among mankind which was supposed to have been introduced from the Low Countries. A famine prevailed, and extensive disease among all animals, but particularly cattle. Even dogs suffered greatly.

"I690. Italy. "On March I3, I observed an epidemic among dogs at Anda of an anginous character. After a very misty night, domestic and sporting dogs, besides three others, all perished in the town, of which, out of curiosity, I took twenty-one for examination, and I found their necks swollen externally and their fauces internally, while the muscles of their throats were much inflamed. Wirth ascribes the losses among animals in Italy to anthrax.'

"I7I4. In March of this year, canine distemper raged in all the southern provinces of France as an epizoöty complicated with gangrenous angina.

" I715. In Germany and France an epizoöty of gastrobronchitis raged amongst dogs.

"1735. Alloa, who was a résident in South America until 1746 , is the first author who has told us of the existence of, and describes the distemper of, dogs of that country.

" 176 I. In this year there began a great epizoöty amongst dogs, which appears to have been what is since vulgarly termed 'distemper.' Before this period it appears to have been very rare or almost unknown, and since its outbreak at that time, it has lingered among the canine species to this day. It seems to have been first noted in Spain. This year there was observed at Madrid a deadly epizoöty among 
gradually fallen into disuse in favour of the universally popular term-Canine Distemper.

During the seventeenth and eighteenth centuries, the word "distemper" was indiscriminately used to signify almost any epidemic of man or epizoötic of beast, though the meaning of the term. has gradually narrowed down to indicate exclusively the specific disease now under consideration. Among the various writers, both English and foreign, there appears to exist a considerable conflict of opinion as to whether distemper originated first in this country or was imported into Great Britain from the Continent. According to one author, a serious epizoötic raged in France about I740, and we learn from others that this was followed, about ten years later, by an outbreak in Germany, and in 1760 by one in England; it then appeared to sweep northwards into Russia, Siberia, Norway, Sweden, and Greenland, assuming in the last-named country a most virulent form upon its first appearance in 1859 .

In the works of some of the ancient observers and authors, we may find vague allusions to outbreaks of epizoötic diseases among dogs in various parts of the world as far back as the year A.D. 1028; but whether in the absence of any detailed description of the symptoms we could safely ascribe the outbreaks as due to distemper is very doubtful. On consulting Fleming's "History of Animal Plagues," the following interesting facts are elicited :

A.D.

"I028. In the present year an invasion of cicadæ and caterpillars in Bohemia following a very plentiful harvest. Innumerable swarms of butterflies appeared, so that everything green in garden and field or in woods was devoured. Dense and foulsmelling vapours had preceded this visitation, rising, as they did, about Easter. After these insects had 
A.D.

eaten everything up, they themselves increased the stench ; the trees also, stripped of their leaves, died and rotted. As a consequence, there was a great mortality amongst men and animals, but especially in dogs in the autumn. England and Gaul, and, indeed, the whole of Europe, suffered in the same way and from the same causes.

"I4I4. According to Saxo Grammaticus, a severe form of dysentery ravaged Germany, affecting horses, cattle, dogs, and cats, as well as man.

" I603. Very inclement season in London, and pestilence among mankind which was supposed to have been introduced from the Low Countries. A famine prevailed, and extensive disease among all animals, but particularly cattle. Even dogs suffered greatly.

"I690. Italy. "On March I3, I observed an epidemic among dogs at Anda of an anginous character. After a very misty night, domestic and sporting dogs, besides three others; all perished in the town, of which, out of curiosity, I took twenty-one for examination, and I found their necks swollen externally and their fauces internally, while the muscles of their throats were much inflamed. Wirth ascribes the losses among animals in Italy to anthrax.'

"I 7 I4. In March of this year, canine distemper raged in all the southern provinces of France as an epizoöty complicated with gangrenous angina.

“I7I5. In Germany and France an epizoöty of gastrobronchitis raged amongst dogs.

“ 1735. Alloa, who was a resident in South America until I746, is the first author who has told us of the existence of, and describes the distemper of, dogs of that country.

"I 761 . In this year there began a great epizoöty amongst dogs, which appears to have been what is since vulgarly termed 'distemper.' Before this period it appears to have been very rare or almost unknown, and since its outbreak at that time, it has lingered among the canine species to this day. It seems to have been first noted in Spain. This year there was observed at Madrid a deadly epizoöty among 
A.D.

dogs, which spread over the whole kingdom, but without affecting any other species of animals.

" 1763 . According to Webster, goo dogs had died at Madrid alone, and in this year it is supposed that the malady had reached England, where many years after it was studied by Mr. Darwin and Mr. E. Jenner. The latter, writing in I809, said: 'It may be difficult perhaps to ascertain the period of its first appearance in Britain. In this and the neighbouring counties I have not been able to trace it back much beyond the middle of last century.'

"I 764. It appears to have reached England at a later period, as Dr. Rutty notices this plague amongst the dogs at Doneraile, co. Cork. The symptoms are a great discharge of a gleety humour from eyes and nose, a difficulty of breathing, violent beating of the heart, also convulsions, and a great weakness in the back and hind legs."

It is comparatively only recently that veterinary schools have come into existence, the first one being established in France in 1761; thus it is fair to assume that, prior to that date, little serious attention could have been bestowed upon the study of epizoötic diseases, and scanty knowledge gained as regards their characteristics. Dr. Jenner also wrote: "My situation in the country favouring my wishes to make some observations on this singular malady, I availed myself of it during several successive years among a large number of foxhounds belonging to the Earl of Berkeley; and from observing how frequently it has been confounded with hydrophobia, I am induced to lay the result of my enquiries before the Medical and Chirurgical Society.... I knew a gentleman who, about forty-five years ago, destroyed the greater part of his hounds, from supposing them mad, when the distemper first broke out among them, so little was it then known by those most conver- 
sant with dogs. On the Continent I find it has been known for a much longer period; it is as contagious among dogs as the small-pox, measles, or scarlet fever among the human species; and the contagious miasmata, like those arising from the diseases just mentioned, retain their infectious properties a long time after separation from the distempered animal. Young hounds, for example, brought in a state of health into a kennel where others have gone through the distemper, seldom escape it. I have endeavoured to destroy the contagion by ordering every part of a kennel to be carefully washed with water, then white-washed, and finally to be repeatedly fumigated with the vapour of marine acid, but without any good result."

Analogous Diseases in Human Beings.-To-day, distemper is ubiquitous the world over, and must be regarded as the greatest enemy of the canine race, among which it causes a high annual mortality, and the loss to dog owners and breeders of many thousands of pounds, apart from the sentimental values which are inestimable in terms of cash. A great analogy exists between distemper and human measles, in that they are both infectious, mostly attack the young, and occur generally only once in life. In each we also find similar symptoms, such as catarrh of all mucous membranes (except of the intestines in measles) and skin eruptions, whilst they are both subject to bronchitis, pneumonia, ophthalmia, and nervous disorders, as complications.

Communicability to Man.-Although this disease has been likened to measles, influenza, and small-pox of man, it has in reality no connection with any of them, and I have heard of no case in practice in which any pathological condition has been communicated to man from animals suffering with distemper. On this question of communicability to mankind, Dr. Jenner might be again 
quoted as follows: "Fortunately this distemper is not conveyed to man. Neither the effluvia from the diseased dog nor the bite have proved in any instance infectious; but as it has often been confounded with canine madness, as I have before observed, it is to be wished that it were more generally understood; for those who are bitten by a dog in this state are sometimes thrown into such perturbation that hydrophobic symptoms have actually arisen from the workings of the imagination. A gentleman who received a severe bite from a dog, soon after fancied the animal was mad. He felt a horror at the sight of liquids, and was actually convulsed on attempting to swallow them. So uncontrollable were his prepossessions, that it was conceived he would have died, had not the dog which inflicted the wound been found and brought into his room in perfect health. This soon restored his mind to a state of tranquillity. The sight of water no longer afflicted him, and he quickly recovered."

However, Dr. M Gowan records several cases in which laboratory workers have contracted symptoms of nasal catarrh after prolonged handling of animals affected with distemper.

I quote below an extract from M'Gowan's paper upon a "Laboratory Epidemic of Distemper," which appeared in the Journal of Pathology and Bacteriology, vol. xv. (I9I I) :

"I. Laboratory workers (five in all)-December, I9og.-Symptoms were catarrh of nose. Cultures made from their noses; organism not found.

“2. Laboratory worker-January, I9ro.-Catarrh of nose. Cultures made; organism not found.

"3. Two laboratory workers-January, I9ro.-Catarrh of nose. Cultures made; organism not found.

"4. Four laboratory workers-June, r910.-Catarrh of nose. Cultures made; organism not found.

"5. Laboratory worker-June 16, I910.-Constantly handling rabbits and guinea-pigs (taking rectal temperatures). Since commencing to 
work in the laboratory-one and a half years ago-this worker has suffered from the worst chronic catarrh of the nose he has ever had. It is very intractable, and at times there are acute exacerbations. Cultures were made from a mass of muco-pus hanging down over the soft palate. At the time the culture was made there was an exacerbation of the condition. A pure culture of the organism without any contamination was obtained."

From these cases it will be observed that only once was the distemper organism recovered from the lesions, and even then no pathological symptom worse than nasal catarrh was set up by it.

In the paper quoted above M'Gowan further says :

"Whether this condition is of common occurrence in man I am unable to say. My observations on individuals in the laboratory would seem to indicate that it is not. That it exists there is no doubt, and there is every opportunity for it to be widespread, considering the number who keep dogs and cats. A kennel-man of forty years' experience told me, without my asking leading questions, that he had had distemper. several times."

Excepting M'Gowan's assertion, I have never before heard it suggested that distemper could be transmitted to man, and under natural conditions I feel convinced it never is.

Distemper as an Entity or Otherwise-Many of the old writers considered that distemper had no individual entity, but it was composed of several affections which may exist either concurrently or separately. In the tenth, revised, edition (192I) of Dorland's "Medical Dictionary," we find distemper defined as follows: "A name of several infectious diseases of animals ; especially a contagious catarrhal disease of pups caused by a specific bacillus. Colt distemper is the same as strangles."

Gray (Wallis Hoare's "System of Veterinary Medicine") remarks: "Although there may be several specific morbid entities included under the generic term of distemper, there are as yet no reliable data to distinguish one from the other, and until bacteriological research, in 
conjunction with clinical study, settles beyond any dispute the question of the unity or multiplicity of affections included in this generic term, the various manifestations of it should be accepted as belonging to distemper." He is of opinion, as a result of a comparative study of the diseases of swine, dogs, and horses, that distemper is an analogous disease to swine fever and swine plague on the one hand, and to influenza, strangles, and contagious pneumonia of equines on the other.

Schantyr, in 1892, claimed that distemper could, by careful bacteriological examination, be divided into three separate diseases-namely, (I) true distemper; (2) abdominal typhus; and (3) typhoid. He added, however, that clinically and anatomically all three were alike.

Mégnin advocated division into two affections-namely, "strangles" and "distemper"; whilst in I90 Cadiot and Breton described two conditions-the true distemper characterised by the presence of an exanthema, and an independent infectious broncho-pneumonia.

Many supposed it to be a true "small-pox," though the complete failure of all those who strove to produce immunity by vaccination seems to have effectually negatived that view. It is, however, not generally looked upon in this light to-day, and although the symptoms of one case may be distinct from those of another, yet there is practically always one combination of symptoms common to all-namely, nasal or bronchial catarrh, or both; cough; and the persistent irritating discharge from the eyes.

Seasonal Predominance.-Distemper may appear at any time of the year, especially in cities, where large numbers of dogs are constantly intermixing. If any period is favoured more than another, it is the warm spring and autumn months, and particularly warm wet weather alternating with cold. 


\section{CHAPTER II \\ SUSCEPTIBILITY}

Animals Susceptible.-The dog is undoubtedly most receptive of the infection of distemper, the cat being susceptible in a much lesser degree; and observers have stated that foxes, jackals, wolves, hyænas, and monkeys are also liable to attack, though I-in common with most other veterinary surgeons-have had no opportunity of verifying the latter assertion or recording any cases among the wild animals.

I have nevertheless come in contact, on several occasions, with sickness among ferrets used for rabbitingthe symptoms of which have simulated those of canine distemper-and even among the rabbits themselves. It may perhaps be assumed that the primary infection in these cases has been disseminated by the farm dog employed in rabbiting operations.

Several foreign bacteriologists have stated positively that canine distemper may be transmitted experimentally from the $\operatorname{dog}$ to the cat and vice versa, and Laosson performed ninety-eight inoculation experiments on dogs and cats, which appeared to show (I) that canine and feline distemper are identical; (2) that they are reciprocally transmitted; (3) that young dogs and cats became infected almost without exception; (4) that mature and old cats and dogs were less susceptible; (5) that the nasal discharge loses its virulence in fourteen days; and (6) that the contents of the pustules were ineffective for the purposes of the experiment.

In spite of these experiments, however, it has been my experience that a cat will not naturally contract distemper 
from a dog sick with it. I have always regarded it as just as safe to allow a healthy cat freedom to wander in the proximity of distempered dogs, as it would be fatal to permit a healthy young dog to follow in its footsteps. Other British observers appear to concur in this view, the contention being that cats are not naturally prone to contract the disease from dogs, though they might do so under artificial conditions.

Herbivorous animals, such as the horse, ox, sheep, and goat, are immune; as are also pigs and birds.

Influence of Age and Breed on Susceptibility.-The breed and age of a dog certainly appear to exert an influence on its susceptibility to attack, and whilst distemper may manifest itself at any period of a dog's life (even as may measles in the human subject), yet mature or aged dogs possess a far greater immunity than do the younger ones. This, however, may be accounted for by the fact that few dogs-if any-ever reach middle life without having already fallen a prey to the disease, and they have acquired therefrom such a degree of immunity that subsequent attacks are averted, or at least assume a milder type. Professor Williams, in his "Principles and Practice of Veterinary Medicine," remarks : "It affects the system only once." But contrary to this and the popular view, the protection conferred by the original attack is not necessarily of a reliable and enduring character, and quite 6 per cent. of those of my patients which I have been able to trace have fallen victims to a subsequent attack.

In rare instances, two and even three attacks have been observed in the same dog, and death has been known to supervene on the second or third illness. In buying a $\mathrm{dog}$, the full assurance that it is over distemper is therefore no guarantee whatever that the malady will not appear again, and there is an abundant accumulation of 
evidence in support of this assertion. Nevertheless, it is fortunately true that, in the great majority of cases, one attack confers an active resistance to future infection; thus in purchasing dogs it is certainly wiser to give preference to those which have attained the age of about twelve months, and which in consequence can be assumed to have passed the danger zone.

Age at which Dogs are attacked.-Distemper is most commonly met with in puppies from six weeks to nine months old, and cases have been observed in pups even as young as ten to fourteen days, whilst in rare instances very old dogs have become infected.

It will thus be noted that the disease is most prevalent during the process of dentition.

Many writers declare that certain breeds of dog have a marked natural immunity, and even attempt to tabulate them according to their degree of resistance; but my view is that no breed is exempt, and none can claim ascendancy in this respect over another, from the point of view of "species." If any difference exists, the real reason will be found connected rather with the conditions of life and environment inseparable from members of the supposed stronger breed. For instance, the shepherd's dog is credited with the greatest immunity, followed in order by the terrier, hound, spaniel, pointer, pug, and so on down the list, until we reach the highly delicate pampered pets, such as Pekingese, Pomeranians, King Charles, and Japanese spaniels, griffons, etc.; and it cannot be denied that the so-called "hardy" "breeds withstand infection and endure its ravages with much greater certainty than the delicate breeds, otherwise there could be no such distinctions of health drawn between the various breeds; but put the sheep dog and his like under the unnatural conditions endured by the average pet dog, and he will probably be found to be as 
frail as he was previously robust. Friedberger and Frohner throw further light on the question in the following passage: "The supposition that certain breeds become more frequently affected with distemper than others is probably erroneous, the more likely cause of this apparent susceptibility being the fact that such breeds are more numerous than others. Thus we find in Berlin that more than one-third of all dogs suffering from distemper are pugs, apparently because this breed is at present fashionable, and not because it possesses any special predisposition to the disease." There is yet another explanation, for Gray tells us that "dogs of exotic origin are more liable to become infected than the indigenous or hardy breeds." Probably this is due to the fact that in the countries of their origin distemper is unknown or rarely occurs. The Terra del Fuegian dogs, in whose native country the malady is unknown, rarely survive an attack. The same may be said of the Arctic breeds, such as the Eskimo, Icelandic, and Greenlander. Even those exotic breeds, such as the Japanese spaniel, samoyede, the chow, and borzoi, bred and reared in our country for generations, succumb readily to its ravages - the Japanese more than any other.

Nevertheless, many of our indigenous breeds that have been too much in-bred, and too carefully nurtured, readily contract the infection under adverse influences, and furnish a great death roll. Such are the bloodhound, greyhound, foxhound, and the sporting dogs, among others too numerous to mention. Notwithstanding this, there are several strains of these various breeds, more capable of resisting the inroads of the disease than others.

Several years ago Sir Ernest Shackleton imported about 200 "Huskies" from Hudson Bay, prior to his embarking on one of his voyages of discovery in the 
Antarctic. These dogs were a large powerful type of samoyede, very wild and little used to handling, and when they arrived in England it was my duty to uncrate and examine them and get them into good health and fit condition for their important labours.

Approximately II per cent., however, were found to be subjects of distemper, and several were so severely stricken that they succumbed. Doubtless, had they remained in their own country they might never have contracted the slightest infection, being inured to the type prevailing there.

Youatt observes that "few dogs imported into this country as exotics do well with it"; thus the greater number of the northern dogs brought by Captain Parry were carried off by distemper within a twelvemonth. 


\section{CHAPTER III}

\section{ETIOLOGY}

Ultravisible Virus Theory.-As a result of some experiments carried on by Carré in 1905, and confirmed later by Lignières ("La Maladie des Chiens," Bulletin Soc. de Méd. Vet., 1905), the former became convinced that "the specific virus or essential element of the disease is invisible, passes through the very porous meshes of the bacterial filter, and is not culturable on various media, the latter remaining sterile. The most active virus is that found in the limpid, serous, or watery nasal discharge seen in the early stages of the complaint; it is equally as virulent when obtained from the pericardial effusion after the death of an animal from peracute or rapid distemper. The filtered and largely diluted discharge, or one-hundredth of a cubic centimetre of pericardial serosity-products not containing any stainable or culturable microbe-cause, after three or four days, a high elevation of temperature, rapidly reaching $105^{\circ}$ or more, and lachrymation, followed by coryza, and afterwards by a somewhat hoarse cough, especially when the animal moves about. At the end of eight or ten days, typical vesico-pustules appear on the places of their electionnamely, abdomen and thighs. The lungs become affected ; the nasal discharge, at first slight, serous or sero-purulent, takes on a purulent character, and continues to flow from the nostrils, and the animal dies after a period of from one to three weeks."

The filterable virus or ultramicroscopic theory thus became established, and nearly all authorities still agree in ascribing as the direct cause of distemper a specific 
ultra-visible virus, the exact nature of which has not yet been fully determined.

That these germ-free fluids will, when inoculated, set up a disease corresponding very closely to distemper seems undoubted, and it was the realisation of this fact which first prompted many bacteriologists to attempt to establish a method of conferring an artificial immunity in the healthy by experimentally inducing a benign attack.

Unfortunately they were not very successful.

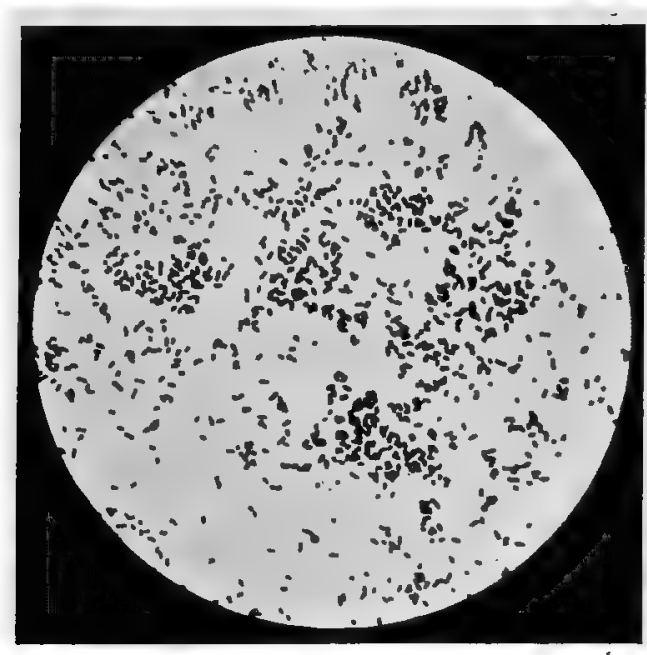

Fig. I.-Bacillus Bronchisepticus.

Isolated by Ferry, and said to be the cause of distemper. Magnified 1,000 diameters.

Bacterial Theory.-Numerous investigators have from time to time isolated various micro-organisms which they have considered to be the specific cause of distemper, but none appear to have been able to convince the veterinary medical world that they have: solved the problem (see note on Bacteriology).

Ferry's Bacillus Bronchisepticus.-However, in October, I908, after four years of continual experimental work, 
Dr. N. S. Ferry, an American veterinary surgeon, isolated the first pure culture of what he contended was the causative factor of the disease. To this organism Ferry gave the name Bacillus bronchicanis, which name he afterwards changed to $B$. bronchisepticus.

Two other workers-namely, Dr. M'Gowan of Edinburgh, and Dr. Torrey of New York-also independently isolated the identical organism, but priority must be given to Ferry, who first described the bacillus in detail in the American Veterinary, Review for July, I9Io, and Journal of Infectious Diseases, J'une, I9 I, and later in the Veterinary Journal, July, 19I4. Ferry found that in the early stages of canine distemper it was possible in almost every case to isolate $B$. bronchisepticus from the smaller bronchi, and frequently from the trachea. At a somewhat later stage of the disease it was found that in the larger bronchi and in the trachea it was contaminated with pus organisms.

He declares that Koch's postulates have been fulfilled, inasmuch as ( $\mathrm{I}$ ) the micro-organism is present and discoverable in every case of the disease; $(2)$ it can be cultivated in a pure culture; (3) inoculation from such culture will reproduce the disease in susceptible animals ; and (4) it can be recovered from such animals and again grown in a pure culture.

Torrey produced the disease experimentally by blowing the dried organisms, or infected dust, into the nasal passages with powder blowers. The symptoms developed included rhinitis and bronchitis, with persistent cough, typical bronchopneumonia, vomiting, bloody diarrhœea, conjunctivitis, and, in three instances, the appearance of a typical pustular eruption of the skin. He concludes:

"The further evidence on which rests the claims that the $B$. bronchisepticus is the etiological factor in canine distemper may be summarised as follows: (I) Dogs 
which are immune to natural distemper show no symptoms when exposed to experimental infection with $B$. bronchisepticus. (2) Dogs actively immunised to $B$. bronchisepticus are immune to natural distemper. (3) Dogs recovering from attacks of experimental distemper induced by this bacillus are protected on exposure to natural distemper. (4) The B. bronchisepticus is the only culturable micro-organism present with uniformity and in great numbers in the tissues and organs of distemper cases."

Granting the accuracy of these statements there appears to be little else to prove, and the first fruits of the discovery were the manufacture of an anti-distemper vaccine and serum, which, whilst not perhaps absolutely infallible, have nevertheless been testified to by a number of veterinary surgeons, dog-breeders, and others, as of considerable value (see "Notes on Prophylaxis").

The first lesson learned by Ferry when he started his investigations was that canine distemper was a disease of secondary infections, and as soon as it was proved to his own satisfaction that he had isolated the primary cause, many questions concerning the symptomatologywhich had previously vexed him sorely-were easily explained. He says:

"From all textbooks and monographs on the subject, I was taught to look to the discharges of the eyes and nose for the etiology; that the disease had been produced by injecting these discharges, either filtered or unfiltered, into healthy dogs. I was struck, however, very forcibly by the fact that, contrary to this, all distempered dogs in the early stages would show some symptoms of an infection of the trachea and bronchi. I at once gave up all work on the eyes and nose, and soon had isolated what turned out to be the cause. As the organism was found in pure culture in practically every case in the trachea, and, in several instances, in the blood, it would seem as though the disease was primarily 
in the respiratory tract, producing often a septicæmia, and in all cases a profound toxæmia. Many of the animals would die with no symptoms other than those of an acute intoxication-namely, a rise in temperature, followed by a sudden fall, loss in weight, and severe prostration, with death within a few days. The relationship of the organism to distemper has been shown by means of agglutination, complement fixation, and other specific tests, so that proof as regards the specificity of the organism is not lacking. It is also of interest to know that this germ will produce a disease similar to distemper in other animals, such as the rabbit; guinea-pig, cat, monkey, and ferret, and when this became recognised, the name $B$. bronchicanis was changed to $B$. bronchisepticus."

Exotoxin Theory.-If this organism is to be regarded as the specific cause of distemper, an explanation may be invited as to how the germ-free pericardial serosity taken from a distempered dog can, when inoculated into a healthy dog, produce identical symptoms. For answer, one is led to conclude that the $B$. bronchisepticus located in the trachea and bronchi generates an exotoxin which circulates in the blood, and which, if individually employed, will set up precisely the same malady as though the actual causal organism were also present. In the various diseases caused by bacteria, changes frequently occur in certain organs which are unassociated with the presence of the bacteria; these are produced by the action of bacterial products circulating in the blood. Two good examples of pathogenic organisms which produce soluble toxins are the $B$. diphtherice and $B$. tetani. In these and similar cases, when broth cultures are rendered germ-free by a bacterial filter, toxic fluids are obtained which, on injection into susceptible animals, reproduce the highly characteristic symptoms of their corresponding diseases.

Exotoxins may be distinguished from endotoxins, 
among other reasons, by the fact that they commonly exert a specific action on particular organs, and even as the bacterial toxins of diphtheria and tetanus exert their influence on the nervous system, so apparently also does that of $B$. bronchisepticus on the respiratory organs. Another almost constant feature is the occurrence of a period of incubation between the moment of infection of the animal and the appearance of symptoms. In contradiction to this exotoxin theory, however, it must be recorded that $M^{\prime}$ Gowan has expressed it as his opinion that no exotoxin is elaborated by the $B$. bronchisepticus.

Filtration Experiments with Bacillus Bronchisepticus.In the year 1915 Ferry, realising that the bacillus in question was a very minute organism, determined to prove, if possible, its ability to pass through the pores of filters used for the demonstration of the presence of known filterable organisms: The only difficulty encountered was in finding a proper and satisfactory means of determining the integrity of the filters. The usual test - that of proving their ability to retain micro-organisms of ordinary size-was obviously out of the question, as the $B$. bronchisepticus is one of the smallest of known organisms. A test known for several years, and well described by Bulloch and Craw (1909), was finally decided to be the most reliable. This test depends upon the measure of the pressure of air as it is allowed to pass through the pores of the filter while immersed in water. A leakage at any point, or a variation in the texture or thickness of the walls, can be detected at once, the efficiency of the filter measured to a fraction of a pound, and the porosity of various filters compared, merely by observing the pressure necessary to produce a general flow of air through the pores of the candles proper. 
The results of the work proved conclusively-according to all rules as laid down by the several authorities on filterable viruses-that the $B$. bronchisepticus is a filterable organism. The work also corroborated the results of previous investigators with regard to the fact that the less pressure used, the more easily will some organisms pass through the filters. Since 1905, when Carré claimed that he had produced typical symptoms of distemper in susceptible dogs from the filtered discharges of diseased dogs, the majority of writers (as already stated) have classified the etiology of canine distemper as a filterable, invisible, or ultramicroscopic virus, and it is so described in many textbooks.

The work of Ferry, M'Gowan, Torrey, and Wherry with the $B$. bronchisepticus tended to refute the statements of Carré, especially as their work was carried on at the same time and quite independently, thus lending great weight to their claims. The work of Carré, however, is not entirely disregarded, many still accepting his position; but the results of the filtration experiments with the $B$. bronchisepticus might appear to put an entirely new light on the subject, as it could be argued that if the $B$. bronchisepticus is the cause of canine distemper, then the experiments corroborate the work of Carré. If the work of Carré is correct, and if the causative agent of distemper is a filterable virus, then the experiments point very conclusively to $B$. bronchisepticus as the cause, and confirm the findings of the above-named investigators.

There is, however, a serious objection to this otherwise lucid explanation-namely, that the filtrates of Carré were said to contain no discoverable or cultivable organism. Thus, granting that Ferry's bacillus could pass through the filter, it should still be possible to cultivate the organism by inoculating an artificial medium with 
the filtered liquid. It would, nevertheless, be interesting to know whether (as Dr. Copeman remarked to me) any British bacteriologist has confirmed Carré's work.

In his paper, included in the Transactions of the Tenth International Veterinary Congress, London, Carré made the statement that he observed seven dogs and was unable to find the $B$. bronchisepticus. He may have examined the dogs in the later stages of the disease, when the organism in question is overrun with secondary invaders, and is not so often found. Ferry's, M'Gowan's, and Torrey's conclusions were drawn as the result of examining several hundred dogs, to say nothing of all the other laboratory animals infected with the bacillus worked on during the past thirteen years; and after reading their articles and accounts of the technique employed, one cannot but appreciate the extreme precautions taken to exclude all outside sources of infection, and the important point that their work was carried on independently, neither knowing that the other was working on the disease. This must mean something to one who is open-minded and looking for facts.

Arguments against Ferry's Organism and Replies Thereto.-Several arguments have from time to time been advanced against the probability of Ferry's organism being the specific cause of distemper: (I) That it is a saprophyte normally inhabiting the respiratory tract of healthy dogs ; (2) can only be regarded as a secondary invader, merely setting up a broncho-pneumonia under favourable circumstances; and (3) that it is not responsible for all the typical symptoms of true distemper.

Broncho-pneumonia itself is obviously not necessarily distemper, and may be regarded only as a secondary symptom of that disease, and the inference remains that an invisible virus is the primary invader, which, having 
weakened the animal's resistance, thereby opens the door to the secondary invaders. Observers have stated that the $B$. bronchisepticus can usually be discovered in the respiratory passages of most healthy dogs, though I think this argument would not preclude the possibility of it being the primary invader; for its pathogenic power to set up symptoms would be enhanced as soon as the animal became debilitated owing to the agency of predisposing causes, such as starvation, bad hygiene, cold, etc. The case of tuberculosis is analogous, for we know that the tubercle bacillus may frequently be found inhabiting normally healthy people without producing lesions of tuberculosis.

Ferry's answer to the assertion that B. bronchisepticus is a saprophyte normally inhabiting the respiratory tract of healthy dogs, and which can only be regarded as a secondary invader, is :

"When this same objection has been brought up in discussing some of my papers . ... I have always asked the critics if they ever found the organism normally themselves, or knew of anyone else, and the answer has been in the negative. B. bronchisepticus is not a normal inhabitant of the respiratory tract of healthy dogs, but these dogs are 'carriers.' I have autopsied between four and five hundred dogs, both healthy and diseased, and speak from experience. I have had carriers in vaccinated animals and in animals that have been housed with distemper dogs, but in the ordinary healthy $\operatorname{dog}$ I have not found the organism. The disease has been produced experimentally with this organism-there is no doubt about it; and dogs have been protected against infection with both live and dead organisms injected subcutaneously-there is no doubt about that either; could this be done with an ordinary saprophyte normally found in healthy dogs?"

In the same letter he dealt with the supposition that $B$. bronchisepticus sets up a broncho-pneumonia which 
might have no connection with typical distemper, as follows :

"Broncho-pneumonia is not the only pathological lesion induced by the experimental injection of B.bronchisepticus. I have seen all stages of the disease produced, from a slight cough and diarrhœa to a case of distemper with all the sequelæ, due to secondary infections, and even a general septicæmia, where a pure culture of $B$. bronchisepticus was isolated from the heart's blood. Torrey, according to his publications, has had practically the same experience. He used the nasal route of injection, while I used the tracheal."

Analogy to Swine Fever.-The whole case seems to be analogous to that of swine fever, the etiology of which is universally declared to be a filterable virus, in spite of the fact that in every pig dead of the disease the Bacillus suipestifer can be demonstrated in its lesions. Although regarded like the $B$. bronchisepticus as only a secondary invader, it is nevertheless a fact that this organism, if fed to healthy pigs, will produce a typical attack of swine fever, even as $B$. bronchisepticus will set up typical distemper, and in neither case is the presence of the virus essential. It is equally true that whereas each virus when inoculated into its respective animal will produce its particular disease, the $B$. suipestifer can be unfailingly recovered from lesions in the pig, and the $B$. bronchisepticus from those in the $d o g$, thus apparently proving that the organisms are constantly and significantly associated with these maladies. Swine fever, as experimentally produced by feeding the culture of $B$. suipestifer, is, however, not naturally contagious to other pigs, and confers no immunity, and we have yet to be assured that the distemper provoked in one dog by artificial inoculation of Ferry's bacillus is infective to other dogs by cohabitation, and after a period of illness leaves them permanently protected against future attacks 
of the disease. (4) When I suggested to Dr. Ferry that a $\operatorname{dog}$ suffering from distemper as a result of experimental inoculation with $B$. bronchisepticus would not be a source of natural infection to healthy dogs his reply was :

"What difference does it make so long as the disease was produced? If distemper is the result of the experimental inoculation, that proves conclusively the specificity of the organism. There are several examples one might cite where animals experimentally inoculated with an injection are not sources of natural infection to other animals. It is a common laboratory occurrence! However, I am not so sure that a dog experimentally infected with distemper, using $B$. bronchisepticus, is not a source of infection. There are no references in the literature that I have run across to that effect, and I have had several cases where I felt sure that they were sources of infection. This criticism, on the face of it, is no argument against the specific nature of the organism."

Copeman's Bacillus.-Dr. Monckton Copeman, F.R.S., isolated a cocco-bacillus in $1900,{ }^{*}$ which he claimed was the specific causal organism of distemper, and he made a vaccine for prophylactic purposes, which some users are agreed affords a valuable protection, not only in warding off attacks, but in rendering those which are contracted very much more benign. [This will be discussed at greater length under "Preventive Inoculation."] There seems some reason to believe that Copeman's organism is very similar to-if not identical with-the one which Ferry isolated in 1908 and described in 1910. The former, however, is said to form frequently long chains (see Fig. 2), whilst the latter is found singly or in pairs. In a letter, Ferry observed :

"While I believe that Dr. Copeman might have been working with $B$. bronchisepticus - and I told him so

* Proceedings of Royal Society, vol. 1xvii. 


\section{ETIOLOGY}

personally when I had the pleasure of meeting him in London in 1914-yet the organism he described was not identical with $B$. bronchisepticus. According to his published description, his organism liquefied gelatin (see p. 32), which puts it entirely out of the class with $B$. bronchisepticus, as B. pertussis, B. abortis, B. melitensis, and $B$. alkaligenes are as close to $B$. bronchisepticus as that."

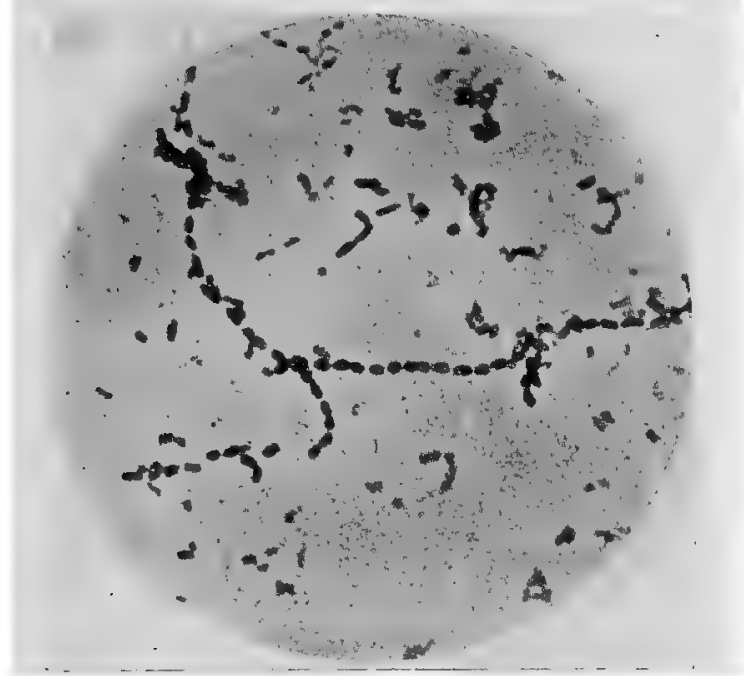

Fig. 2.-Dr. Copenan's Bacillus, showing Chain Foriation.

From a pure old culture in beef-peptone broth. $\times$ I,500. Proceed. ings of National Veterinary Medical Association, 1902.

Copeman obtained a few isolated colonies of his organism from the base of the brain in the case of a dog which had died of distemper. The fact of his finding these discrete colonies, few in number, at the base of the brain, along with the fact of the other situations in the bodies of distempered dogs where he found his organismthe fact that he never obtained it from the blood, and the cultural appearances on potato-disposed Dr. M'Gowan, in later years, to consider Copeman's organism was identical to his own.*

* Journ. Path. and Bact., IgIt. 


\section{METHODS OF INFECTION}

As regards the means by which an animal naturally becomes a recipient of the contagium, we may safely rule out inoculation, and regard that as almost exclusively confined to the research-laboratory; we are thus left with two other methods-namely, inhalation and ingestion-and there seems little doubt but that the disease is contracted both ways.

Cases are known in which healthy susceptible dogs have accidentally gained access to the food and water of a distemper patient, which had undoubtedly been contaminated with infective discharges, and as a result of partaking of the meal have contracted the disease.

This, in addition to the undisputed fact that rubbing the nasal or ocular discharge of a diseased animal over the lips or nostrils (from which it is licked) of a healthy dog will produce distemper in the latter, should be sufficient proof that ingestion is a frequent natural method of infection. My personal view, however, is that the majority of cases of distemper arise through inhalation of the contagium, either by droplet infection or by aerial infection.

In the former method, the bacillus or its toxin is conveyed through the atmosphere as, or in, small droplets of sputum which have been thrown into the air during the act of coughing or sneezing, and which remain suspended for some time. By aerial infection, the contagium is transported through the air by means of small dust particles. Distemper can be as easily spread in this way as can tuberculosis, through the drying-up and dissemination of sputum, nasal discharges, etc. Blaine said in $185 \mathrm{I}$ : ". . . even being exposed to the air impregnated with the exhalations from a distempered dog for a few minutes is sufficient for the purpose." 
Unfortunately the dog habitually has its nose to the ground; he smells everything everywhere, and in consequence becomes especially prone to the baneful effects of injurious dust, etc. How else can we account for distemper being caught by a dog, which, in its innocence and curiosity merely runs into a kennel, stable, or room in which a distempered animal had previously been; runs out again without touching the walls or eating anything, and in a few days falls sick? How did Sir E. Shackleton's dogs become infected if not by inhalation, as they never left their crates (except on the ship) between Northern Canada and England, and yet about twenty-two were subjects of distemper upon arrival? Nevertheless, Youatt was correct when he remarked: "There is a great deal of caprice about the way in which a dog will or will not become infected, for I have more than once kept a dog in the foul yard of my hospital for several successive weeks, and he has not become diseased."

Messrs. Sewell, Gray, Parsons, and Flook are all of opinion* that distemper is seldom, if ever, communicated from the affected to the non-affected by aerial infection. The contrary view is held by Friedberger, Frohner, Blaine, Glass, Müller, myself, and various others, and whoever may be correct, it is at least a wise precaution to regard it as transmissible by aerial infection.

This chapter would not be complete were particular stress not laid upon the fact that distemper can only arise from the presence of its specific contagium, and that a cold will not and cannot produce it. As a contributory or predisposing factor, chills among other conditions are forces to be reckoned with, and will be discussed in the section dealing with predisposing causes. One writer declared that "whilst distemper is highly contagious, it

* Proceedings of National Veterinary Association. 
is yet frequently generated, and in this bears an analogy to mange, and to farcy and glanders in the horse." Such a theory as "spontaneous generation," however, is quite exploded, for all the maladies mentioned can only be created by their respective specific parasites or organisms.

Latent Infection.-There is, of course, such a contingency as latent infection, in which an animal appears to harbour the contagium and disseminate it to others, without itself showing any manifestations of disease whatever. Such an animal is said to be a "carrier," and he may remain so for at least three months, and possibly even longer, after recovering from distemper, especially if there had been middle ear or frontal sinus complications. 


\section{CHAPTER IV \\ BACTERIOLOGICAL NOTES}

ONE of the first to write intelligently at any length on the subject of distemper was Jenner, who in I809, while recognising the true infectious nature of the disease, was the first to differentiate between distemper and rabies, and also the first to show that it was not communicable to man. Later this was disputed by many of the best writers.

Since that time many have been actively engaged in attempting to discover the etiology of the disease, and of them all, the primary investigator to discover an organism was-

Semmer, who in 1875 isolated a particularly short and narrow bacillus in the blood of sick dogs a few hours after death. He concluded this was the causal organism, and was strengthened in his opinion by-

Laosson's researches in 1882, in which a micrococcus and a bacillus were found, and cultivated in broth ; with the mixed culture he claimed to have transmitted the disease.

Krajewski, in I88 I, observed micrococci in tissues and blood.

Rabe (I883) found uniform globules of minte size, sometimes lying together in heaps (staphylococci), or connected in twos or fours (diplococci), yor in rows of four or five (streptococici), stainable dark blue with methylere viotet $\rightarrow \mathrm{He}$, however, could not reproduce the disease; ant Friedberger, who confirmed his findings, left the specificity of the organisms an open question.

Mathis (1887) found a diplococcus in the fluid of the 
tissues, sputum, and pustules, which he cultivated in neutral or slightly alkaline broth. Pure cultures were made, which, on injection into healthy susceptible dogs, set up catarrhal and pustular symptoms accompanied by acute pyrexia, and followed sometimes by death of the animal. Survivors, however, had acquired an immunity.

Marcone and Meloni ( 1888 ) isolated staphylococci which, when cultivated and inoculated into dogs, set up pustules but no typical distemper.

Jacquot and Legrain (I890) found in the pustules motile micrococci which were from 0.6 to $0.8 \mu^{*}$ in diameter, and which formed diplococci; but inoculations of their cultures produced no better results than Marcone did with his organism, and brought about no immunity. The coccus found by Mathis was thought by Lignières, Carré, and others to be identical with this one, and it was considered to be the direct cause of the pustules.

Millais (I 89o) found in lungs, liver, trachea, spleen, and nasal mucus a long bacillus which, on inoculation, set up typical distemper, and which liquefied gelatin, descending as a flaky mass in the almost clear fluid, which was covered by a whitish scum. Finally, when the whole of the gelatin was liquefied, the flakes gathered at the base, and, if stirred, appeared to be of a ropy consistency. He also found a micrococcus in great abundance, which he thought was the cause of the lung lesions. The two combined caused the pneumo-distemper (Veterinary Journal, May, I 890).

Schantyr ( I 892) claimed to have found three organisms, and divided distemper into three diseases, each produced by a distinct organism (see p. 7).

Jensen ( 1896 ) declared that the pneumonia of distemper was due to a streptococcus, which he demonstrated.

Valerio (1896) found in the pustules, spinal medulla,

$$
\text { * } \mu=\text { microns. }
$$


brain, and lungs an ovoid bacillus $0^{\circ} 3 \mu$ wide by $1 \cdot 20$ to $2.5 \mu$ long, motile, Gram-positive, causing gas production in gelatin stab, cloudiness in broth, and not coagulating milk. Inoculation with this bacillus produced symptoms of distemper, particularly of the nervous type.

Babes and Barzaneseo (1897) isolated a motile bacillus in two cases. Seven dogs out of nine inoculated died of distemper in about two weeks.

Petropawlowsky (1897) found a bacillus similar to Bacillus coli.

Taty and Jacquin (1898) discovered a diplococcus in the central nervous system, which they regarded as the cause of the nervous form of distemper.

Jess ( 1899 ) cultivated a bacillus ( $\mathrm{I} \cdot 8$ to $2 \cdot 3 \mu$ long by 0.6 to $0^{\circ} 9 \mu$ wide) from the nasal discharges, blood, and peritoneal fluid; Gram-positive, and forming a dull grey film on agar and a white one on potato; found to be pathogenic to dogs, cats, and guinea-pigs. $\mathrm{He}$ considered his organism dissimilar to any of those hitherto discovered, and also stated that he was able to produce distemper experimentally with it.

Copeman (1900) found a cocco-bacillus in the nasal secretion, tracheal mucus, and exudation from the lungs, which, in smears from broth, not infrequently formed chains, sometimes of considerable length ; Gram-negative, and growing readily on agar at $36^{\circ} \mathrm{C}$. in greyish glistening circular colonies; it grows well in broth, which first is turbid, but later a deposit falls and the liquid clears; growth on serum slow; occasionally gives a moist yellowish growth on potato; gelatin liquefied and milk not coagulated. Inoculations of dogs with living cultures produced attacks, occasionally fatal, of distemper. In one instance in which the bloodvessels of the brain were found to be much congested, inoculation of a tube of sloping agar with a platinum loopful of cerebro-spinal 
fluid, well spread over the surface of the agar, resulted in the appearance of half a dozen isolated colonies of a pure culture of his bacillus. He never succeeded in obtaining cultures from the blood.

In a recent interview with Dr. Copeman, he explained to me that, although in his original paper read before the Royal Society in December, I900, he stated, "If gelatin be inoculated, growth occurs slowly at room temperature, and after a time the medium tends to become liquefied," he was, in fact, misled by the great heat of that summer, and that subsequent observations proved that gelatin was not liquefied.

Lignières and Phisalix (I900-I90I) found a long bacillus which they concluded was Pasteurella canina (an organism similar to $B$. avisepticus), cultures of which, when inoculated into susceptible dogs, produced typical clinical signs of distemper-e.g., catarrh of the nasal and conjunctival mucous membranes, broncho-pneumonia, pustules, gastro-enteritis, corneal ulceration, chorea, paralysis, etc.-the malignity of the attack being modified according to the dose. The organism was a Gramnegative, non-motile one, which assumed a cocco-bacillary form in guinea-pigs, and both authorities testified to its presence in the heart's blood of dogs in acute distemper, though they were unable to isolate it in nasal and other discharges. Phisalix has often found the Pasteurella in very long filamentous forms, which have all the cultural, staining, and virulent characters of typical Pasteurella, though they become gradually attenuated after several sub-cultivations, and with age. Passage through the dog, however, restored their former virulence. Phisalix prepared from its cultures an immunising vaccine, which he and Lignières declared gave protection against distemper. Copeman tested it and found that it was in many cases ineffectual against infective matter prepared from 
the organisms alleged to be the cause of the malady. The Pasteurella canis was freely criticised by several authorities, Jensen considering it was not the cause of distemper, and McFadyean, Richter, Vallée, and Carré stating that it merely set up an accidental infection. Phisalix came to the conclusion that it generated an exotoxin which, by disturbing nutrition, weakened the animal's

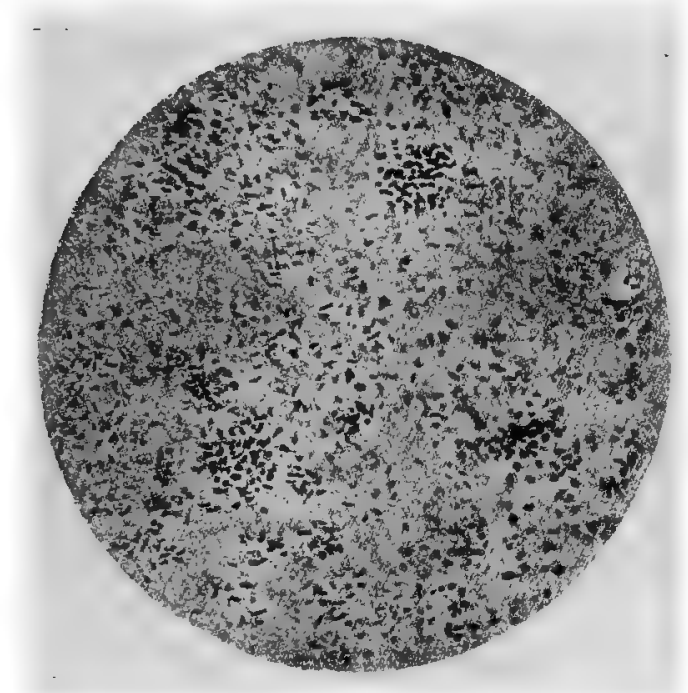

Fig. 3.-Pasteurella Canis of Lignieres and Phisalix. $\times$ i,ooo. Proceedings of National Veterinary Medical Association, I902.

natural defensive powers and facilitated secondary infections. Indeed, it is an opinion shared by many observers, including myself, that animals succumb to these secondary infections more often than to the primary disease.

Cadiot and Breton (I9OI) pursued some investigations, as a result of which they concluded that the infectious broncho-pneumonia was a disease quite independent of distemper proper.

Von Wundscheim (1905) discovered an organism of the hæmorrhagic septicæmia type which he named 
B. canicidus, pure cultures of which produced the usual typical symptoms of distemper. Its habitats were the nasal discharge, spleen, and blood.

Carré (1905), by his numerous experiments, satisfied himself (and a considerable following) that the contagium of distemper was a powerfully pathogenic filterable virus, which could be found in the early watery nasal discharges and in the serous effusions of the pericardium, peritoneum, and pleura of dogs which had rapidly died of the complaint. He found that 2 or 3 drops of either of these fluids would induce typical distemper symptoms and frequently death; that filtrates contained no discoverable or cultivable organism, and that animals infected experimentally would pass on the contagion to healthy animals, and would-if they survived-be immune to further attacks.

Bose (1905) concurred in this view, and expressed his opinion that the virus was composed of very minute protozoa, and that, in consequence, distemper should be classed as a protozoal disease.

Hewer (1906) found a short, small, non-motile, Gramnegative bacillus giving a fine white growth on agar, slightly clouding broth with granular sediment and stringy masses, and not liquefying gelatin.

Kregenow (1909) raised a dissenting voice; he considered the watery discharges were not infective, and that the primary cause of the malady was not an ultravisible virus.

Ferry (r9Io), after long experiment and research, isolated the $B$. bronchisepticus, which, after subsequent investigation, he satisfied himself was the specific cause of distemper. He states:

"A bacillus was found which grew slowly and with difficulty when first isolated from the body, so that extreme care was necessary when looking for the 
colonies. At the end of twenty-four hours it was often impossible to see anything on the surface of the agar without the use of a lens. The colonies appeared as pin points among the larger colonies of other bacteria, and at times it was very difficult to isolate the bacillus on account of the overgrowth of other organisms. Blood cultures were taken as a routine measure at all autopsies, and at first nothing grew but the organisms of the secondary infections. Later, however, the method was changed. The heart was opened aseptically, and from I c.c. to 5 c.c. of blood drawn off with a pipette and planted in a flask containing 50 c.c. of broth, or distributed in several tubes of broth. After twenty-four hours in the incubator, plates were made, or slope agar inoculated in successive tubes. Such blood cultures were taken in 63 cases. In 29 the cultures were positive, the bacilli being found in 18 , in 13 of which it was uncontaminated, in 3 associated with staphylococci, and in 2 with unidentified bacteria. The remaining I I positive blood cultures had staphylococci only in 6, staphylococci with an unknown bacterium in $I$, and unidentified bacteria in 4 cases."

The characteristics of the organism were:

Short slender bacillus, $0^{\circ} 5$ to $2^{\circ}{ }^{\circ} \mu$ by $0^{\circ} 5 \mu$, found singly or in pairs; sporeless, flagellated, actively and progressively motile; Gram-negative, and not staining readily with ordinary stains, but with Loeffler's methylene-blue shows a characteristic bipolar appearance; no liquefaction of gelatin or coagulation of milk or gas formation. After twenty-four hours' incubation on agar, there is a moist dew-drop growth, which later becomes larger and opaque; bouillon cloudy; older growth with heavy stringy sediment ; potato tan; light tan in twentyfour hours to dark tan in three weeks; medium also becomes tanned.

When cultures are taken early in the disease, the $B$. bronchisepticus is found in the respiratory tract in every case, and if taken in the first stage is found 
uncontaminated. The organism was found in the larynx, trachea, large and small bronchi, lungs, and blood.

M'Gowan and Torrey (I9II) had been working independently of each other, and simultaneously with Ferry, and both isolated what was agreed to be the identical microbe found by Ferry. M'Gowan's bacillus was taken from the nasal and tracheal mucous membrane in all cases, from empyemata of the middle ear, from meninges (chorea cases), and lungs (pneumonia cases); never in the blood, the condition thus not being of a septicæmic nature. It was found to be pathogenic to dogs, cats, rabbits, monkeys, ferrets, and guinea-pigs. Its characteristics were: Gram-negative, non-sporulating, feebly motile, flagellated; no acid, indol, or gas formation; gelatin not liquefied; and on glycerine agar it assumes an almost coccal form (Journ. Path. and Bact., vol. xv., I9II). Torrey did not publish his observations until I9I 3 (Journ. Med. Res., vol. xxvii., I9I3), when they substantially agreed with all that Ferry and M'Gowan had previously said.

The Great War doubtless curtailed further concentrated effort during the succeeding years, for up to the moment of writing, no new theory or discovery has been propounded on this most complex question of etiology. Investigations are, however, now being actively pursued in this and various other countries, and the fervent hope and ambition of all concerned is that a conclusion may, ere long, be formulated which will admit of no denial, and be accepted by all.

The conclusions to be drawn from a review of the observations of the above-named investigators is that the organisms of Ferry, M'Gowan, Torrey, Copeman (in view of his statement regarding the non-liquefaction of gelatin), Semmer, Laosson, Babes and Barzanesco have a marked similarity - at lèast morphologically, if 


\begin{tabular}{|c|c|c|c|c|c|c|c|c|c|c|c|c|}
\hline 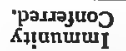 & & & & & & & & + & + & + & + & + \\
\hline 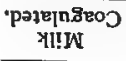 & & 1 & & & 1 & & & 1 & 1 & & 1 & 1 \\
\hline $\begin{array}{l}\text { pajnpord } \\
\text { ses }\end{array}$ & & + & & & + & & & 1 & 1 & & $i$ & 1 \\
\hline 'ฆ[โวธิย[ี & & & & & & & 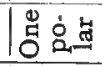 & & 1 & & + & + \\
\hline 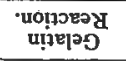 & & 1 & & + & 1 & & & 1 & 1 & & 1 & 1 \\
\hline 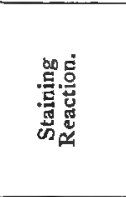 & . & 点荥 & है & & 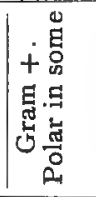 & 焉 & 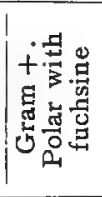 & 韷 & | & & 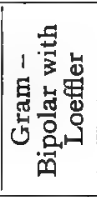 & 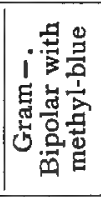 \\
\hline 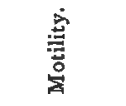 & & $i$ & + & & + & & + & + & 1 & 1 & + & 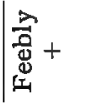 \\
\hline 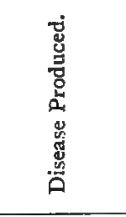 & 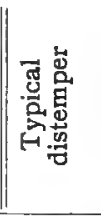 & 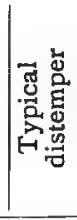 & 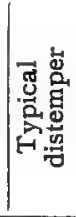 & 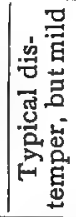 & 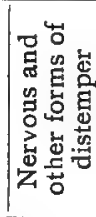 & & 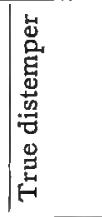 & 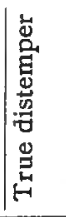 & 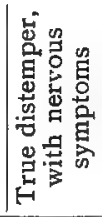 & 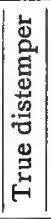 & 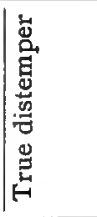 & 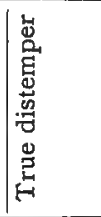 \\
\hline 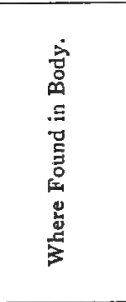 & 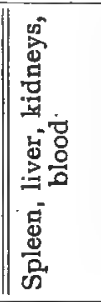 & 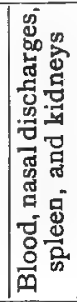 & 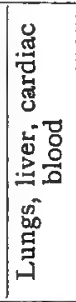 & 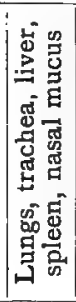 & 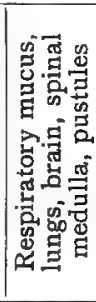 & & 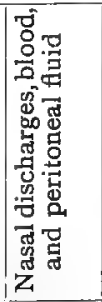 & 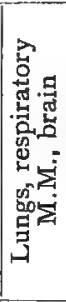 & 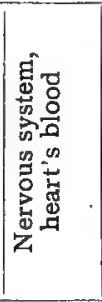 & 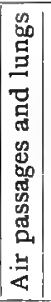 & 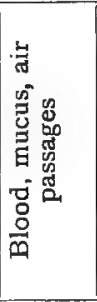 & 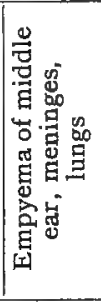 \\
\hline 'selods & & 1 & 1 & & + & & & 1 & 1 & 1 & 1 & $!$ \\
\hline 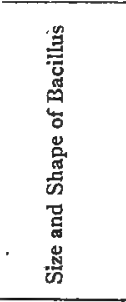 & 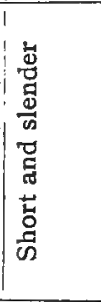 & 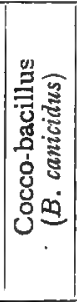 & 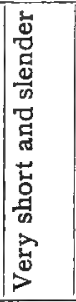 & 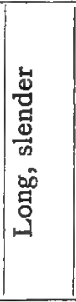 & 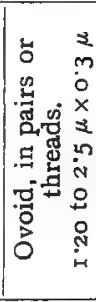 & 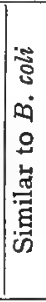 & $\begin{array}{l}3 \\
2 \\
9 \\
0 \\
x \\
z \\
0 \\
0 \\
i \\
0 \\
0 \\
\infty \\
i\end{array}$ & 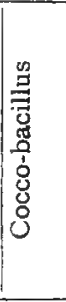 & 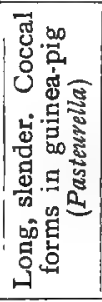 & 总 & 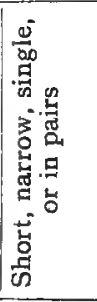 & 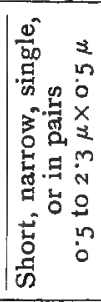 \\
\hline 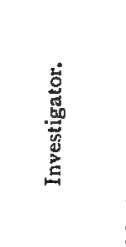 & 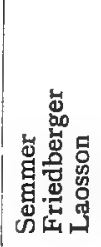 & 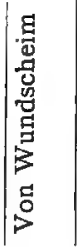 & 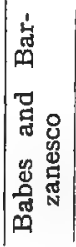 & 䄈 & 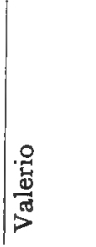 & 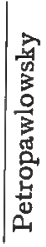 & $\stackrel{\mathscr{D}}{\mathscr{W}}$ & 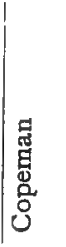 & 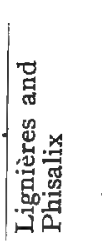 & 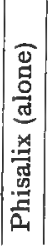 & 意 & 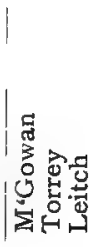 \\
\hline
\end{tabular}


not culturally-for upon reference to the table on p. 37 one finds that in all cases inoculation was claimed to have produced the true disease, followed by immunity; all were cocco-bacilli, or very short slender organisms; all could be demonstrated in the blood (except Copeman's and M'Gowan's); all were motile and non-sporulating; all Gram-negative; and, where records are available, none coagulated milk.

Is it not possible-nay, even probable-that they are one and the same organism? Referring to the table on micrococci (p. 39), it is evident that all authorities except Mathis are in agreement that they do not set up the typical manifestations of distemper, nor is immunity conferred; they are regarded purely and simply as secondary organisms, though doubtless very pathogenic, and probably responsible for a greater mortality than the specific organism.

It is noteworthy how few of the organisms were discoverable in the cerebro-spinal system, the only examples being those of Copeman, Taty, Jacquin, Valerio, Phisalix, and M'Gowan, and in all cases the predominant symptoms produced were of the nervous variety; yet when M'Gowan, Sabrazès, and Muratet endeavoured to cultivate growth of any organism from the cerebro-spinal fluid of dogs and cats afflicted with paraplegia, their results were entirely negative.

Secondary Invading Organisms.-Whilst admitting the difficulty of being able to assert with absolute precision that any particular organism is the prime factor in the etiology of genuine distemper, we are definitely aware of the existence of a multiplicity of germs playing a secondary, though important, rôle in the development of the disease. On account of the irregularity and infrequency with which these organisms are isolated, producing upon inoculation only local lesions or those of 
a pyæmic character, one concludes they have no connection with the primary infection. As the distempered

\begin{tabular}{|c|c|c|c|c|}
\hline Investigator. & $\begin{array}{l}\text { Organism } \\
\text { Discovered. }\end{array}$ & $\begin{array}{l}\text { Where Occurring } \\
\text { in Body. }\end{array}$ & $\begin{array}{c}\text { Disease } \\
\text { Produced. }\end{array}$ & $\begin{array}{l}\text { Immunity } \\
\text { Conferred. }\end{array}$ \\
\hline Semmer & Diplococci & & & \\
\hline Laosson & Diplococci & & & \\
\hline Millais & Micrococci & $\begin{array}{c}\text { Nasal secre- } \\
\text { tion }\end{array}$ & $\begin{array}{c}\text { Pulmonary } \\
\text { complications }\end{array}$ & \\
\hline Phisalix & Streptococci & $\begin{array}{l}\text { Lungs and } \\
\text { pleural effu. } \\
\text { sion }\end{array}$ & & \\
\hline Ferry & $\begin{array}{l}\text { Streptococci } \\
\text { Staphylococci } \\
\text { Very small } \\
\text { micrococci }\end{array}$ & $\begin{array}{l}\text { Eye lesions } \\
\text { Blood }\end{array}$ & $\begin{array}{l}\text { No typical } \\
\text { distemper } \\
\text { lesions }\end{array}$ & None \\
\hline Krajewski & Micrococci & $\begin{array}{c}\text { Tissues and } \\
\text { blood }\end{array}$ & & None \\
\hline $\begin{array}{l}\text { Rabe } \\
\text { Friedberger }\end{array}$ & $\begin{array}{l}\text { Staphylococci } \\
\text { Streptococci } \\
\text { Diplococci }\end{array}$ & $\begin{array}{l}\text { Nasal dis- } \\
\text { charge } \\
\text { Ocular dis- } \\
\text { charge } \\
\text { Pustules }\end{array}$ & $\begin{array}{c}\text { Distemper not } \\
\text { transmitted }\end{array}$ & None \\
\hline Mathis & $\begin{array}{l}\text { Diplococci } \\
\text { (I to } 3 \mu \text { dia- } \\
\text { meter) }\end{array}$ & $\begin{array}{l}\text { Sputum } \\
\text { Tissues } \\
\text { Pustules }\end{array}$ & $\begin{array}{l}\text { Similar symp- } \\
\text { toms to those } \\
\text { of distemper, } \\
\text { but chiefly } \\
\text { pustules }\end{array}$ & $\begin{array}{l}\text { Immunity } \\
\text { conferred }\end{array}$ \\
\hline $\begin{array}{l}\text { Marcone } \\
\text { Melone }\end{array}$ & Staphylococci & & $\begin{array}{c}\text { Only pustules } \\
\text { produced }\end{array}$ & None \\
\hline $\begin{array}{l}\text { Jacquot } \\
\text { Legraine }\end{array}$ & $\begin{array}{c}\text { Motile diplo- } \\
\text { cocci } \\
\text { Streptococci }\end{array}$ & In pustules & Only pustules & None \\
\hline Jensen & Streptococci & & $\begin{array}{c}\text { Pneumonic } \\
\text { symptoms only }\end{array}$ & None \\
\hline $\begin{array}{l}\text { Taty } \\
\text { Jacquin }\end{array}$ & Diplococci & $\begin{array}{l}\text { Nervous sys- } \\
\text { tem }\end{array}$ & $\begin{array}{l}\text { Nervous } \\
\text { symptoms }\end{array}$ & None \\
\hline
\end{tabular}

Fig. 5--Micrococci associated with Distemper and Regarded AS SECONDARY INVADERS.

dog practically always suffers from one or more of these secondary infections, and since these are often the 
greatest difficulty with which we have to contend, it is rather important to have some idea of them, and the conditions under which they are found.

Many of the earlier observers apparently confined their attention to the purulent discharges which were thought to have been occasioned by the primary infection, and they found chiefly staphylococci-especially albus, though sometimes aureus-these being constantly present in all purulent affections. Towards the end of an acute case this coccus may also be sometimes demonstrated in the blood, the animal probably dying from the terminal staphylococcic infection. Streptococci have occasionally been observed in conjunctival discharges, lungs, pleural effusions, and pustule contents, and have been blamed as the cause of pneumonic lesions of distemper. Diplococci have been found in sputum, nervous system, tissues, pustules, etc., and have been held accountable for production of abscesses and the cutaneous and nervous symptoms. Several bacilli have been isolated from nasal and other discharges, bronchi, lungs, spleen, liver, kidneys, blood, etc., which, if inoculated subcutaneously, often produce an œdematous swelling or abscess at the site of inoculation, and even death; and in these cases the organism may be isolated in pure culture from the blood, though that is far from proving that they are the primary cause of the disease. In the lastnamed organs Ferry often found a large bacillus, which he believed to be a terminal invader; "for dogs dying when this organism is present always have a putrid, cadaverous odour several days before they die, and after death the surrounding atmosphere becomes almost unbearable."

Practitioners who have dealt much with fatal distemper will doubtless readily remember having encountered this stench; it could not be forgotten. A diphtheroid 
bacillus has been isolated from eye secretions, and another from the lungs of dogs dying from typical symptoms of distemper, but the organisms proved to be non-pathogenic for dogs or other animals.

The superintendent of a well-known English research laboratory informs me that the organisms isolated from a large number of cases of distemper were:

In discharge from the eyes one or more of the following-Staphylococcus aureus and albus, Streptococcus brevis, a diphtheroid bacillus of $B$. kerosis type.

In discharge from the nose one or more of the following-Staphylococcus aureus and albus, B. coli communis, Pneumococcus, Streptococcus maximus, B. bronchisepticus.

From post-mortem cases all the following organisms: B. bronchisepticus, B. coli communis, Pneumococcus, Streptococcus brevis and maximus. 


\section{CHAPTER V}

\section{PREDISPOSING CAUSES}

As previously emphasised, nothing whatever can cause distemper except exposure to its specific contagium, but many circumstances can arise which tend to render the animal more susceptible to its reception. It is universally accepted that any condition which tends to weaken or debilitate the animal, impairs the natural defence of the body, and reduces its power of resistance to the invasion of pathogenic organisms, which are perpetually seeking a suitable soil upon which to grow. The human or animal body only becomes a suitabłe soil when its resistance has been broken down, and the invader is victorious.

Influence of Breed and Age.-Breed and age have a marked influence on predisposition, and, as pointed out in the chapter on "Susceptibility," age particularly plays an important part, young dogs being much more prone to contract the malady than old. Similarly, highly-bred and delicate pampered lapdogs offer very little resistance to the infection, and when attacked in puppyhood large numbers succumb to it. The mongrel has ever proved itself to be the stouter animal in disease, and the more remote its breed is from the pure, the greater seems its power of resistance and recovery.

Influence of In-breeding.-In-breeding exerts a weakening influence on the constitution, and is too frequently responsible for rickets, deformities, alopecia, sterility, and mental incompetence; thus such an animal, robbed of its vital faculties, becomes an easy prey to disease, which probably terminates fatally. 
Influence of Acclimatisation.-Recently imported or unacclimatised dogs are particularly predisposed to distemper, since it is a fact that dogs born and bred in one locality or country appear to acquire a certain immunity to the one or more strains of infection which contaminate its surroundings, but when moved into an entirely new area become subjects of the disease.

Influence of Faulty Hygiene.-Crowding many animals together, even under good hygienic conditions, but especially when associated with careless management, permitting of insufficient space and ventilation, and disregard of thorough cleanliness and disinfection, prove a sure method of courting the contagium. Similarly, lack of exercise, unnatural systems of feeding and rearing, starvation, fatigue from super-abundant or enforced exercise, or from prolonged travelling by rail or sea, all tend to lower the system, and render the animal more susceptible.

Influence of Chills and Debilitating Conditions.-Probably the chief predisposing cause is chill, brought on by exposure in various ways-e.g.: (I) A dog may be playfully thrown into water, presumably to teach it to swim ; it emerges wet, and shivers in a cold wind. (2) It may be bathed and insufficiently dried. (3) It may be inadvertently locked out in the open all night. (4) Or a wretched yard dog may occupy a leaky, bedless, or draughty kennel in severe wintry weather. But whatever the cause, the result is the same, and should the dog at this time come into contact with the infection of distemper, he may contract the disease, notwithstanding the fact that he may have previously suffered from it.

Then we have as contributory factors the debility arising from the ignorant use of purgatives and vermifuges, and, less frequently, that following severe surgical operations, parturition, and prostrating diseases. 
Seasonal Influences.-Finally, there are seasonal influences to be contended with, for dogs-like human beings-are more prone to fall ill in moist, muggy, and changeable weather than they are in fine warm or even fine cold weather. This is presumably why distemper is more prevalent in the spring and autumn, seasons which are fickle and trying. The exceptionally dry warm summer experienced during I92 I did not by any means increase the number of cases of distemper, as might have been expected by some observers, but my experience was that it occasioned a noticeable decrease.

In Youatt's opinion distemper frequently follows mange; "in fact, if he treated such a case of skin disease, he would certainly expect the dog to contract distemper as a general routine." There is, however, no connection whatever between the two maladies, and the only justification for Youatt's assertion is that mange is frequently a debilitating disease, and might thereby figure as a predisposing cause like any of the other circumstances mentioned above. 


\section{CHAPTER VI}

\section{SOURCES OF INFECTION AND THEIR PRACTICAL AVOIDANCE}

In indicating the multiplicity of means by which this bighly contagious disease is spread among dogs, it is anticipated that owners will be materially enlightened as to the steps it is desirable to adopt in preventing infection, not only of their own dogs, but among "the whole canine race. Forewarned is forearmed, and if one is cognisant of the pitfalls, they can usually be avoided. The most fruitful source of infection is direct contact with a living animal suffering from distemper; almost as potent, however, is mediate or indirect contact, accomplished through the agency of contaminated kennels, baskets, rugs, clothing, brushes, leads and collars, sponges, feeding utensils, spoons, thermometers, human agency and clothes; or, in fact, any article whatsoever which, having been in the immediate proximity of a distempered dog, becomes a source of great danger to the healthy. It is the discharges, both ocular and nasal, but particularly from the nose, and the coughedup sputum, which are so infective. Especially is this the case in the early stages of the disease, and when they become smeared in even the most minute quantity upon the ground or on such objects as are mentioned above, they are likely to be conveyed over wide areas, and become the invisible means of spreading the contagion to innumerable animals. Therefore, prompt and efficient measures for disinfection of inanimate objects, and the strict isolation of affected animals, must be adopted. 
I have long since learned to regard with the greatest suspicion any catarrhal condition of the dog simulating what is popularly termed "cold in the head," or "snuffles," or "relaxed bowels," and contend that there is little, if anything, which distinguishes an ordinary chill (non-infective) from specific distemper in the early stages.

If all dog owners would be equally suspicious, and would isolate a dog immediately it manifested the slightest symptom, many a serious outbreak would be avoided. There are, nevertheless, numbers of people who adopt this procedure; then finding after several days that the animal appeared to regain its normal liveliness and commence to feed, they permit it to mix again with the other dogs, only to discover later that the slight malaise and huskiness had in fact been distemper in benign form, and that the convalescent dog had infected the rest of the kennel through being put back too soon.

Duration of Infection after Convalescence.-Infectivity still exists for a time during convalescence, and in estimating the time which must elapse after an attack before a dog can be stated to be free from infection we must take into consideration the date upon which the very last trace of any symptom whatever was noticedsuch as cough, discharges, diarrhœa, or temperature-at which time the dog might be pronounced as completely restored to health. Then a week should be allowed to pass to guard against the possibility of relapse, and if still well and bright, the patient might receive a disinfectant bath with due precautions, thereafter being safely assumed as germ-free.

Disposal of Contaminated Articles.-On the termination of an attack of distemper, all old brushes and other articles which have been used on the sick dog should be burned, if they do not lend themselves to thorough disinfection. 
Mediate Contagion.-In many cases people take the wise precaution to isolate an ailing dog, but forget that in attending to its wants the kennelman is liable to carry infection on his hands, boots, or clothing to other dogs. Either one man must devote his sole attention to the sick, or a system must be devised of donning overalls and goloshes before entering the infectious ward, removing them on leaving, and also washing the hands. The disease being so highly infectious, it is absolutely essential to study these details, as half-measures are doomed to failure in this as in most undertakings.

Dog Shows.-Dog shows are undoubtedly a prolific source of contagion in spite of veterinary inspections, for, as already explained, a dog may be a "carrier"i.e., have the infection latent in its system-whilst to all outward appearance in perfect health, or may be passed in by a veterinary inspector during the "period of incubation"-i.e., before visible symptoms have manifested themselves. A proportion of these "carriers" can be detected by taking the temperatures.

Veterinary Inspections at Dog Shows.-My personal experience of veterinary inspections at shows is that, as at present conducted, they are a farce. I suppose the majority of exhibitors are conscientious enough to desire their dogs to be examined on entrance, though a goodly proportion, I fear, regard the whole thing as a waste of time, and unless carefully observed they will slip in without a word. Should the examining surgeon detain or debar a dog, however, even those of the conscientious class become highly indignant, and proceed to protest vehemently that "the journey upset it," that " it is only a cold," or "it is always like that," or any other conceivable excuse; and where the veterinary surgeon remains adamant, there are frequently altercations and unkind remarks. 
Again, in many large shows there is often only one veterinary surgeon but several entrances, and although guiding barriers and notices are set up to direct exhibitors to him, he cannot attend to everybody simultaneously, and as it is not strictly enforced that each should await his turn, many pass through in the crush unobserved. After all, the examinations can only be of a very cursory nature, and unless any striking symptom is apparent, it would be impossible to give a definite opinion as to whether distemper existed or not in any particular dog. The most that can be accomplished is to exclude any glaring cases of infectious disease, and even these may be overlooked if the veterinary inspector attends as a matter of mere form without any thought of performing a duty.

His position is nearly always an honorary one, and the exhibitors must trust to his conscience and his willingness really to serve their interests. These would undoubtedly be best served if he were paid. Furthermore, it would seem very desirable, if these inspections are to have any practical utility, that the examination of all animals should take place outside the hall, or in an annexe; for if a dog afterwards found to be suffering from distemper is allowed inside before it is inspected and rejected, the mischief is done, and contagion already spread. The gravity of allowing an infected dog into. a show is emphasised when it is remembered that these animals travel from show to show all over the country, and may cause widespread infection, the blame for which might conceivably be attached to an innocent show, person, or dog, quite unconnected with the outbreak.

Exhibitors' Legal Liability. - It should be pointed out that a person who sends, or causes to be sent, to a dog show any animal knowing it to be suffering from an 
infectious disease, exposes himself to the liability of being sued in a court of law for compensation by any other dog-owner who has sustained loss through such wilful neglect.

A Suggestion to the Kennel Club.-In the interests of the dog-owning public, no less than of the dogs themselves, these entrance examinations should be compulsory, and admission of a dog to the hall should depend upon the possession by its owner of a pass obtained from the veterinary inspector. The latter, on his part, should make a careful and conscientious survey of each dog, and if the number of entries be beyond his power to cope with, assistance should be provided. Since his hands must necessarily pass from dog to dog, a wise precautionary measure would be for him to dip them frequently into a strong disinfectant solution without drying them.

The adoption of rigid measures and the observance of detail are really the only sane methods of attempting to suppress this calamitous disease, and mitigate the great financial losses annually sustained. To labour the necessity for cleansing and disinfecting show-rings and benches is probably superfluous, as that is generally recognised and well carried out. The contingency of a visitor or exhibitor attending a dog-show having recently been in contact with a case of distemper at home is not to be overlooked, and for this reason the handling of exhibits by the public should as much as possible be discouraged.

Risks Attending Brood Bitches, etc.-The system of dispatching brood bitches from all parts of the country to stud dogs for mating purposes must inevitably assist in perpetuating the disease, and it would be difficult to suggest any measure calculated to overcome this danger entirely. The demand by both parties of a guarantee that 
no infectious disease exists, or has recently existed, in either establishment would probably assist materially, though that is open to some doubt.

Risks Attending Dogs "on Approval."-A similar objection attaches to the practice of sending dogs on trial or approval, for they have been frequently observed to fall ill soon after their return home. As previously stated, a dog usually acquires immunity after his initial attack. In other cases, where a puppy grows up in a contaminated environment, it frequently becomes immune to the predominating infection of its own kennel without exhibiting at any time more than the most transient indisposition; but expose this same dog to a new strain of contagion and it readily falls a prey, or will in turn (although considered quite healthy) transmit a virulent attack to strange dogs.

The only precautionary measure practicable is rigidly to isolate the brood bitch immediately upon her return from stud, and for the owner of the sire, after departure of the bitch, to do likewise for at least two weeks. Dogs returning from "approval" should be similarly treated as suspects before allowing them to mix again with the other inmates of the kennel. Although this would not avoid the incidence of disease in the animals concerned, it would at least prevent an outbreak of serious dimensions throughout the whole establishment; entailing, most probably, considerable loss of time and great inconvenience, yet in the end it would prove a very much cheaper and wiser policy.

Public Highways.-A constant source of infection are the public highways, parks, etc., where dogs of all breeds are perpetually exposed to both mediate and immediate contagion. If a $\operatorname{dog}$ is to be allowed its freedom to run in the streets there would seem to be no way of protecting it except by the somewhat uncertain 
agency of vaccines (see p. 58). Hounds and other sporting dogs, despite the fact that they cover considerable areas of the countryside, are not exposed to the same risk as the town dog, although it must not be supposed that the country is free from infection-far from it. When, however, one hound becomes affected, it is a serious matter, in view of the great probability of a rapid spread throughout the whole kennel, at any rate among the younger members of it. Thus, again, we find the need for strict isolation of those hounds which, having been placed out with farmers and others, are eventually returned to the master in the spring.

Curtailing a General Outbreak. - Though such hounds may appear in the pink of health, it is very inadvisable to place them immediately with their companions, as, should a latent infection exist, a serious outbreak would probably result. In a case where the disease gains entrance undetected to a kennel containing a number of dogs, owing perhaps to carelessness in not isolating new-comers, the procedure is to remove the infected dog immediately, place it in strict isolation, and segregate all "in-contacts"-i.e., separate from the remainder all dogs which are known to have come in contact with the infected.

If these cannot be determined owing to indiscriminate mixing, it is then advisable to group the dogs, allowing no members of one group to mix with those of any other. Should distemper appear later in one of the groups, the infected can be jsolated, and the "in-contacts" segregated as before, and a general outbreak will possibly have been avoided. During these proceedings the temperatures of all dogs should be taken daily, and any rise over IOI $5^{\circ} \mathrm{F}$. regarded with great suspicion.

Essentials of Isolation.-For isolation to be of real utility, the following points should be observed : 
I. The dog should have no direct or indirect contact with any other of its species.

2. Exercise should be taken alone on ground used by no other dog, and no opportunity should be afforded for sniffing at other dogs through railings, gates, or windows, etc.

3. The attendant should not have charge of any other dogs, or, if this is impracticable, he should, before discharging his duties to them, cover his clothing and boots with overalls and goloshes, and should wash his hands frequently with disinfecting fluid.

4. No other person should visit the dog for fear of conveying infection from the suspected dog in isolation to other healthy animals.

5. Food and water utensils, brooms, and all other articles should be strictly confined to the isolation kennel, and not mixed with others or washed in the same water with others, nor used indiscriminately for other animals.

6. Soiled bedding, sawdust, and excreta should be removed and burned, or placed where no other dog can have access to it.

7. Frequent disinfection should be observed.

Dog Bureaux.-To purchase a dog from the average dog bureau is really courting trouble, since no shop can truthfully be declared as free from infection. Any place at which fresh dogs are continually arriving is bound to be contaminated sooner or later, in spite of the most rigorous hygienic measures; and where the management is in the least apathetic or careless, the evil grows in proportion. In course of practice it is my duty to inspect daily certain establishments of this kind, and although the greatest vigilance is exercised, there are discovered occasionally dogs which are manifesting signs of the malady, necessitating their instant removal 
and the routine labour of scrubbing and disinfecting their pens, and sterilising implements. Thus, unless rank carelessness can be proved, it is hardly fair to blame the shop should a dog develop distemper shortly after purchase; it is the expected result and the risk must be accepted.

Dog Homes.-Many people, in the hope of "picking up a bargain," bestow their patronage upon one or other of the homes for lost or starving dogs. In these cases, if a dog is purchased, it is a foregone conclusion that distemper has been bought too-with hardly an exceptionunless the animal is middle-aged and possessed of an active immunity.

To consign one's pet to any of these charitable institutions, to be boarded during the owner's pleasure, is equally risky, even though the boarders may be located in specially provided quarters well away from the stray dogs. A more commendable plan would be to choose a reputable boarding establishment, preferably where veterinary examination is available and no distemper cases permitted on the premises. Veterinary infirmaries and private sanatoria or boarding establishments which refuse to accept any animal suffering from, or under suspicion of, distemper will-if conducted on sound principles-have a system of isolation by which every new-comer will undergo observation in a special ward for three weeks, before being moved into the main building or allowed to use the same exercising ground as other dogs of proved health.

In further pursuance of this plan, a special kennelman is required, whose duties and instructions preclude him from attending to or mixing with any animals other than those under observation. To ensure further the noncommunication of possible infection from one ward to another, measures should be taken to destroy all vermin, 
particularly rodents (see Appendix). For the same reason, separate food and water dishes, bedding, biscuits, brushes, harness, and other gear should be kept for these isolated dogs, that they may be, as it were, self-supporting, and assume a separate entity. Notwithstanding all these precautions, however, I have known a dog to contract distemper even after it had been three months in quarantine, no case of the kind ever having been on the premises, and the quarantined dog never having left them. When the possible source of infection was sought, we were entirely at a loss to fathom it, and could only conclude that the dog was a "carrier," or that infection had reached it by way of the atmosphere from another carrier. Under ordinary conditions it is extremely rare for dogs in quarantine to become subjects of distemper, inasmuch as they live an entirely separate existence.

Well-kept Kennels. - The premises of a veterinary surgeon who really caters for dogs need never be the source of contagion believed by some, for his kennels will be tiled, or of brick faced with cement, thus lending themselves to easy and thorough cleansing; by reducing woodwork to a minimum in the construction of the kennels, absorption of urine, with its consequent evil smells, will be obviated; efficient ventilation will be provided without permitting draughts, and the atmosphere will be kept at a healthy and constant temperature of about $60^{\circ} \mathrm{F}$. A system of examination and isolation of each dog admitted will ensure no chance contact between the sick and healthy, and a veterinary surgeon who specialises in canine practice and gives his whole time to dogs will go round his wards frequently and observe the progress and condition of his patients. Sunlight is one of the greatest enemies of all micro-organisms, and its adequate provision should pre-eminently occupy the minds of all who are entrusted with the building of ranges 
of kennels. I.ight and air are among the very few things obtainable gratis nowadays, yet their value should not be depreciated on that account, nor their supply limited.

To Detect Distemper.-Laymen frequently ask, "How shall I know my dog has distemper?" For answer, I would counsel them to treat as distemper all catarrhal or other conditions, until the contrary has been proved. For instance, the symptoms of refusing food, showing indifference or malaise, shivering, creeping near the fire and evincing a disposition to sleep, slight husky cough, the least nasal or eye discharge, diarrhœic tendency, or even a dry, hot nose, would collectively or individually be sufficient ground for suspecting the onset of distemper. Having recognised some such disturbance in the dog's health, the common mistake of waiting for the advent of further and more pronounced diagnostic symptoms should be avoided, and the animal immediately placed in quiet seclusion. A "wait and see" policy in these cases is fatal.

Infective Matter and its Source.-It must be borne in mind that the virulent contagium is to be found in the watery nasal or lachrymal discharge and respiratory mucus, particularly in the early stages. Although it has been demonstrated also in the blood, spleen, kidneys, liver, cerebro-spinal fluid, etc., these are unlikely to have any connection with the spread of infection. There remain as the only other possible sources-the fæces, urine, and pustular contents; but the latter have never revealed any agent which would set up typical distemper, and apparently no observer has recorded the discovery of any important organism in the urine.

In one of Ferry's reports he stated that recent findings had proved conclusively that the symptom of diarrhœea is one of the most important, as the $B$. bronchisepticus may be found in large numbers in the early intestinal dis- 
charges, and the spread of the disease is probably due more to these discharges than to the pus from the eyes and nose. If Ferry's organism can be accepted as the causal one, it would follow that the clinical thermometer may well be condemned as a means of contamination. Konhäuser, whose inoculation experiments had a negative result, is of opinion that the contagium is also in the milk of bitches suffering from distemper; but no other investigator has confirmed this, and its truth seems open to considerable doubt.

Resistance of the Contagium to Cold and Heat, etc.Experimental evidence shows that the contagium is not destroyed by desiccation, although its virulence is considerably attenuated after being kept in a dry condition for several months. Freezing, even to as low a temperature as $-4^{\circ} \mathrm{F}$., does not kill it, but a temperature of $60^{\circ} \mathrm{C}$. renders it sterile. Laosson found that nasal discharge exposed to the open air loses its virulence in from eight to twenty days.

Disinfection.-It would appear highly desirable in a work of this nature to indicate the various types of disinfectants, their mode of action, and suitable choice for the object in view, since the prevention and cure of distemper from beginning to end consists of nothing less than an organised fight against bacterial life. A detailed description of the agents used and methods employed will be found in Appendix II.

\section{PERIOD OF INCUBATION}

The stage of incubation of distemper appears to be very variable, and is undoubtedly influenced by several considerations, such as the virulence of the causal organism, constitution of the animal, prevailing weather conditions, etc. A large number of views have been expressed based on observations following both experi- 
mental inoculation and infection by cohabitation, the date of exposure to infection being known in every case, and the consensus of opinion computes the average period at 4 to 7 days. The earliest appearance of symptoms has been placed at 3 days, and the longest at 3 weeks (Hobday), whilst many other authorities have recorded intervening periods, such as 5 to 8 days (Trasbot), I 2 to I5 days (Nocard and Leclainche). Krajewski found the period of incubation was 4 to 7 days, but he states that cases of infection through cohabitation may sometimes take from 2 to $2 \frac{1}{2}$ weeks to develop. These protracted periods, however, are rare, and personally I would expect to discover the earliest symptom about a week after a susceptible dog had been exposed to the disease. 


\section{CHAPTER VII}

\section{PREVENTIVE INOCULATION}

WHEN resistance to an infection is a natural inborn quality, we speak of it as natural immunity; but it is possible to evoke an acquired immunity by means of inoculation with an organism or its derivatives, just as an ordinary attack of infectious disease confers a certain degree of acquired immunity. Active immunity may be set up by one or more of the organisms concerned in attenuated condition, or by sub-lethal doses of virulent organisms or their toxins, or by inoculations of dead microbes and their products, or even of their products alone. As gradually increasing amounts are injected, the animal will develop a high degree of resistance. This procedure constitutes preventive inoculation or vaccination.

Immunity is, however, not immediately created, the time elapsing being governed by the susceptibility of the animal and the dose and virulence of the vaccine. Though it is comparatively a slow process, the active immunity evoked lasts a considerable time.

In passive immunity protection depends upon the introduction of substances developed in some other animal, and it has a rapidly produced but transient effect. When an animal has been hyper-immunised with increasing doses of vaccine as just described, its blood serum will contain substances which have an antagonistic or neutralising action on the specific bacteria or their toxins, and such a serum will be of value for the prevention or cure of certain infections in another animal. A notable example is anti-tetanus serum. 
Theory of Immunity.-Wright's "opsonic" theory of immunity, briefly outlined, is to the effect that there is a substance (opsonin) present in the serum of an immune animal which, when introduced into another animal affected or likely to be affected with the disease against which the first animal is protected, renders the corresponding germ sensitive to phagocytosis-i.e., peculiarly liable to attack and destruction by the white blood-cells. Thus the opsonic qualities of the serum constitute the means by which the body frees itself from the invading organisms. The occurrence of a low opsonic index is due to the using up of available opsonin, and the object of a vaccination is to supply this deficiency by stimulating the mechanism for its production, and this is the immediate effect of the first injection; but a reaction then occurs, normally lasting a day or two, in which the amount of opsonin is actually lower than it was in the beginning. This is called the negative phase, during which the system becomes more susceptible to disease. When the vaccination is successful, this phase is followed by a rise in the opsonic value to a higher level than that existent at the time of infection. This is known as the positive phase, and the body has become more resistant to microbial invasion. Sometimes the positive phase is quickly and easily obtained, but at others there is a prolongation of the negative phase, and should a second dose of vaccine be then administered, there is a danger of inducing symptoms of the disease to manifest themselves.

In the earlier days it was the practice to inject attenuated living cultures of organisms into the animal to be protected, but now it has been found safer and more convenient to use dead cultures, at least at the commencement; in both the principle is the same, and both cultures are spoken of as vaccines. 
Age at which to Vaccinate.-When our object in vaccinating dogs against distemper is one of prevention and not cure, we need to carry it out as early as possible, in order that the risk of natural infection before the operation may be diminished. On the other hand, it is highly desirable that the pup should have been weaned, and become accustomed to its ordinary food and hardened somewhat to its new life. Thus the suitable age would appear to be seven or eight weeks, though there is no reason why it should not be attempted later in life, except the increasing danger of infection prior to inoculation; for it is well known that if a latent infection exists when vaccination is performed, the addition of a little more toxin to an already contaminated system might overpower the existing defensive forces, and perhaps cause a very alarming disturbance.

The puppy, having received its series of two or three injections, should be isolated for a full fortnight to permit of immunity becoming firmly established, and certainly should have been excluded from all possible sources of contagion during the whole period of his treatment. No doubt unfortunate sequelæ have often attended the exposure of a puppy to infection too soon after inoculation; even exposure to chills or fatigue would render the animal more liable to contagion by the temporary lowering of vitality produced.

Further, to obviate accidents, it is advisable, in cases where the dog is known to have been exposed to the contagium of distemper, to give a dose of immune serum before, or along with, each prophylactic dose of vaccine, to produce an immediate passive immunity. Previous inoculation with serum enables all animals, even those which are in a state of latent infection, to receive vaccine without any danger.

In fact, this method might well be adopted as a routine 
in every instance, and would doubtless reduce to a minimum those regrettable occurrences too frequently experienced in the past when vaccine has been used alone. With the provision of a regular nutritious diet, warmth, and cleanliness, all will have been done that is possible to ensure one's efforts being attended with success.

Reactions during Vaceination.--These may be severe or altogether absent : they may be manifested merely by a local reaction, or the general health may be disturbed. Where there is a moderately severe swelling at the seat of inoculation, the animal is often rendered stiff or even lame, and exhibits perhaps some degree of pyrexia and inappetence. In other cases alarming symptoms are evinced, simulating those of the disease it is sought to prevent, and the animal may even die. In the majority of instances, however, the train of symptoms is usually no worse than loss of appetite, dullness, general malaise, loss of condition, rise of temperature to $102^{\circ} \mathrm{F}$. or 103 ${ }^{\circ} \mathrm{F}$., followed in about seven days by complete recovery.

Most authorities appear to be agreed that the greater the severity of the general reaction the more lasting the immunity conferred, and where negative results have attended vaccination, the cause has often been ascribed to the failure to produce a suitable typical reaction.

In the discovery of a prophylactic or curative vaccine or serum lies the only hope of salvation for the canine race against the dread scourge of distemper, and the bacteriologist who can accomplish this will deservedly become famous in the annals of veterinary science. The first step will naturally be the fixation, beyond a shadow of doubt, of the causal agent, after which the creation of a preventive vaccine could be entertained with a more reasonable assurance of success; but in any case the desired end will prove extremely difficult to attain, in 
view of the fact that even a naturally contracted attack does not in all cases confer an active immunity.

\section{PREVENTIVE INOCULATION}

I. With Vaccine Lymph.-As early as I795, Viborg, attaching great importance to the pustular lesions of distemper, advocated vaccination with cow-pox virus, which procedure was tried on various later dates by other investigators, and renounced as ineffectual. Notwithstanding this, Dr. W. Brown, writing in the Veterinary Journal (November, I902), observed: "For a number of years I inoculated all the puppies at Drumpellier House, Coatbridge, with vaccine lymph, and with the best results. During my time I never heard of a case of distemper arising after inoculation. This was done at the request of Sir David Carrick Buchanan, who was a profound believer in the efficacy of the above treatment. I usually inoculated behind the ear, and no ill results seemed to follow." His observations, however, have met with no support whatever, and such men as W. Sewell, Blaine, and Youatt before him, and A. Sewell, Hobday, and others after him, have expressed no uncertain opinions regarding the utter uselessness of vaccine lymph.

2. With Crude Nasal Discharge.-Another early method was the inoculation of susceptible animals with the crude nasal discharge from a distempered dog, with the object of inducing a mild attack and its subsequent immunity. Unfortunately, however-as might be expected-a large majority contracted a more malignant type of the disease and frequently succumbed, so that the method was eventually abandoned.

3. With Pure Cultures of Bacilli.-Investigations were pursued, and Semmer, in 1875, was the first to discover micro-organisms in connection with distemper. $\mathrm{He}$ 
isolated a micrococcus and a short, slender bacillus from the blood and lungs, and as the bacillus was also plentiful in the spleen, liver, and kidneys, he concluded it was the causal organism. In succeeding years, numerous other authorities discovered bacillary or coccal organisms in various parts of the body, and each considered his own germ as the primary cause of the disease. All endeavoured to obtain pure cultures with which to reproduce the typical disease, and most were successful; but when they attempted to confer a lasting immunity, they unhappily failed, though they sometimes claimed to have succeeded.

4. With a Dead Culture-Copeman's Vaccine-We arrive now at the year 1900, in which Dr. Monckton Copeman described in a paper read before the Royal Society how, by heating a broth culture of the bacillus (isolated first by his confrère Millais twelve years previously, and regarded as the causal organism) to $60^{\circ} \mathrm{C}$. for half an hour, and subsequently adding a small quantity of carbolic acid as a preservative, a vaccine was obtained which acted in a similar fashion to those devised by Haffkine and Wright for use in prevention of plague and enteric fever, respectively, in man. The dose must obviously vary according to the size of the dog, but generally a fox-terrier puppy would receive 2 c.c. of the sterilised culture.

Evidence Supporting the Efficacy or Failure of Copeman's Vaccine.-Dr. Copeman writes personally: "Curiously enough, wide divergences of opinion have been expressed by those who have made trial of the preparation introduced by myself. On the Continent, and more particularly in Germany, hundreds of doses have been employed with, as I am informed, most gratifying results; but in this country I have had the utmost difficulty in obtaining reports. From a member of my own profession, not a 
little to my astonishment, I learnt that he had feared after all to use the vaccine, lest it should actually cause an outbreak of distemper among his dogs, notwithstanding the fact that in my communication to the. Royal Society I was careful to describe the exact method by which my prophylactic is produced, showing that sterilisation of the fluid and the consequent killing of all living micro-organisms is an essential factor of the process. It is doubtless true that any protective influence against distemper which the vaccine is capable of affording is due to the fact that its inoculation gives rise to what is practically a mild attack of the disorder, but with this most important difference from the natural disease-that owing to the method of the preparation of the vaccine it is absolutely impossible for an inoculated dog to infect with distemper other dogs with which it may come into contact. I provided Mr. Sewell with material for testing purposes, but he did not succeed in producing in puppies by its use any marked immunity.

"On looking over the temperature charts of the animals inoculated by him, it is apparent that in no instance did any reaction follow, marked as it would have been by some rise of temperature, and that therefore little or no immunity could be expected to ensue.

"In carrying out inoculations, it is obviously essential that a sufficient dose should be employed if any measure of success is to be obtained. Herr Carl Hopf, in a letter to me, rightly lays stress upon this point, stating that whereas all the vaccinations performed by himself had proved successful, notwithstanding prolonged exposure of the inoculated animals to the infection of distemper, on making inquiry as to certain alleged failures reported to him he found that only about I c.c. of the vaccine had been injected in each instance. In conclusion, I would suggest that where the first injection is followed by no 
obvious reaction, the operation should be repeated after the lapse of a fortnight or three weeks. In this case a double dose of vaccine should be used, and as a matter of precaution the second injection should not be made at the same place as the first."

Twelve months later, however, Mr. Sewell repeated his experiments with Copeman's vaccine, but unfortunately fared no better than upon his first attempt. Messrs. Hobday and Ridler, F. and M.R.C.V.S., carried out an observation, in which six fox-terrier puppies were vaccinated, two of them with Copeman's product, and the results were as follows:

CASE I.-March 23, 3 p.m.: Fox-terrier, male, three months old, fair condition; vaccinated with 45 minims subcutaneously in the thigh. Temperature, IO2 ${ }^{\circ} \mathrm{F}$.

9 p.m. : Very dull, back arched, as if in pain, and cold. Temperature, $1024^{\circ} \mathrm{F}$.

March 24: No appetite; very tucked up flank, stiff, and disinclined to move. Weight, 3 lbs. i 2 ozs. 'Temperature, $103^{\circ} \mathrm{F}$. at 7 a.m. and $102 \cdot 6^{\circ} \mathrm{F}$. at 7 p.m.

March 25: Found dead at 6.30 a.m.

Weight of carcase, 3 lbs. 9 ozs.

CASE 2.-March 23, 3 p.m. : Fox-terrier, female, three months old, good condition; vaccinated by injecting 30 minims subcutaneously inside the thigh. Temperature, $1024^{\circ} \mathrm{F}$.

9 p.m. : Very dull, back arched, off feed, and not inclined to move. Temperature, $104^{\circ} \mathrm{F}$.

March 24: Evidently very ill. Weight, $4 \frac{3}{4}$ lbs. Temperature, $102^{\circ} \mathrm{F}$. Swelling at seat of vaccination.

March 25: Drank a little milk; very lame, and looked very ill. Temperature at 7 a.m., Io ${ }^{\circ}$ F., and at 6 p.m., $103^{\circ} 4^{\circ} \mathrm{F}$. Swelling at seat of vaccination.

March 26: Large wound inside thigh, quite $1 \frac{1}{4}$ inches long, where swelling had burst. Animal went about on three legs. Temperature, $105^{\circ} \mathrm{F}$.

From this date the dog seemed better, gradually acquired use of her legs, and regained her appetite; 
temperature became normal, and wound healed. It required careful attention until July, and even then was very thin, coughing, and showing signs of the severe illness it had gone through; and, as Mr. Hobday aptly remarks: "It makes one, as a practitioner, very careful about giving an emphatic opinion to a nervous client in favour of vaccination as a prophylactic, and an owner will wonder whether the risk is the greater from the remedy or from the disease itself."

Nevertheless, in my opinion, had these vaccinations been preceded by a dose of anti-distemper serum, these grave disturbances would not have been manifested.

Mr. F. B. Carrell, writing in The Kennel, April, s91 I, said: "I have inoculated my dogs with Dr. Copeman's anti-distemper vaccine for nearly five years, and I think if I write my experiences, it may be of assistance to the dog-loving public in helping to arrest the appalling mortality from distemper in present-day dogs. . . . A friend told me of Dr. Copeman's vaccine, and he had two months before inoculated two smooth collie pups, aged five months. At the time a few miles away was a kennel of griffons, all affected with distemper, and many were dying. To find the worth of the vaccine the nostrils of the two vaccinated puppies were pushed against those of the diseased griffons, and some of the mucus from the eyes and noses of the latter was inserted into the nostrils of the inoculated collie pups and plugged with cotton-wool. One collie was not affected at all, temperature normal, appetite good; in fact, perfectly healthy. The temperature of the other collie rose to $103^{\circ} \mathrm{F}$, appetite capricious, and puppy rather irritable. This lasted about a week, after which he became perfectly normal. Both these puppies were afterwards exhibited all over England, and neither has shown any further sign of distemper. ... My next litter of puppies was inoculated at the age of four months. Three pups of the four had a good reaction, by which I mean that at the end of three or four days the temperatures rose to $103^{\circ} \mathrm{F}$, and remained so for about ten days, the patients being irritable and feeding indifferently. The remaining 
pup had no reaction; consequently, at the end of three weeks, I again injected a larger dose, which reacted only very slightly.

"Some six months later I bought (unwittingly) a bitch with distemper, and she ran at exercise with the beforementioned puppies. All these took distemper, but were only slightly ill for about a week (discharge from eyes and nose, slight cough, slight diarrhœa, temperatures from $102^{\circ}$ to $103^{\circ} \mathrm{F}$.), but at the end of the week were perfectly well again. The whole of that litter is alive now ; all have been exhibited, and two are frequently at shows, yet none have ever had any further signs of distemper, and I have several times had distemper dogs in the kennels.

"I think, therefore, in showing that in my own kennels, in the first five years of breeding, without inoculation, the mortality from distemper was at the rate of over 50 per cent., whereas in the last five years, with inoculation, the mortality is only 2 per cent., there is very little room for doubt that we must look to inoculation to help us against the scourge of the kennel-distemper."

About this time Mr. Stewart Wood, M.R.C.V.S., testified to having inoculated sixty-nine dogs with Copeman's vaccine during the past few years, the majority of which only received one injection. To his knowledge only five of them subsequently had distemper, of which two died. This, however, is no reliable or true test, as he does not state whether any of the dogs had already acquired an immunity, nor does he actually know whether any of the remaining inoculated dogs contracted the malady or not. The most recent testimonial which has come into my hands by the courtesy of Dr. Copeman is a letter received by the Jenner Institute in October, 1920, from His Grace the Duke of Beaufort. It runs as follows: "The Duke of Beaufort, on the recommendation of Dr. Monckton Copeman, inoculated his entry of $18 \frac{1}{2}$ couples as they came in from 'walk' last spring (two inoculations), and up to the present time they have shown no symptoms of 
distemper. The young hounds were also inoculated (half dose), and so far no deaths from distemper have been reported. The Duke hopes he may be equally successful this year."

An extract from a further letter from $\mathrm{Mr}$. T. Newman, huntsman to His Grace the Duke of Beaufort, reads: "As soon as the hounds were brought in from 'walk,' they were isolated, fasted for about twelve hours, and given a gentle dose of castor-oil, and then temperatures taken, to make certain they were in a fit state to inoculate. Then they received 7 c.c. of vaccine. Took their temperatures for three days to make sure of the reaction, which in most cases sent their temperatures up from $102^{\circ} \mathrm{F}$. to as high as $104^{\circ} \mathrm{F}$; ; kept them still in isolation for a period of ten days from the first inoculation, then did them again with ro c.c., taking their temperatures for three days. I find that in the majority of cases it reacts the second time. Then I put them together. Out of the $23 \frac{1}{2}$ couples that were inoculated, only about one couple have shown any signs of distemper, and then only in a mild form. If we go on with the same results as this year, it will be the greatest boon that has ever been brought out."

Further testimony as to the value of Copeman's vaccine was also given by Karl Hopf, the well-known St. Bernard breeder, in a letter to The Dog World; but it is phrased in similar terms to the other letters, and on that account, perhaps, need not be recapitulated. M'Gowan, on the other hand, obtained no definitely good results with the use of prophylactic vaccines, though he considers they may prevent toxic phenomena.

Ferry's Polyvalent Polymicrobial Vaccine.-The very latest production is the vaccine introduced by Ferryan American veterinary surgeon, who, as I have fully described under "Etiology," discovered B. bronchisepticus, 
which he and several other workers are positive is the specific causal organism. With several strains of this microbe, a vaccine was made and experiments carried out to test its immunising properties on a large number of dogs, and in a few instances on other animals, such as the Canadian lynx, sea lion, fox, etc. Forty dogs were used in all, nine being immunised with live cultures and seventeen with dead cultures, while fourteen were saved as controls. All of these dogs were exposed to at least three animals suffering with typical symptoms of distemper, including the respiratory, abdominal, and nervous types.

Eight of the controls died, while all the immunised dogs remained well. To substantiate further the position given to $B$. bronchisepticus, Ferry, and also Torrey and Rahe (Jour. Med. Res., vol. xxvii., I913), carried on a relatively large number of prophylactic inoculations with suspensions of the dead organisms, and found in a large percentage of cases that a true protection had been established in the inoculated dogs, and that the controls in practically all cases rapidly succumbed to the ravages of the disease.

Ferry wrote in 1913: "From a practical standpoint, we have found these protective inoculations of very great value, as from the nature of work carried on in our laboratory it is necessary to use from fifteen to twenty dogs a week for one purpose or another, and it has always been our experience to lose nearly all of the young ones with distemper. Since beginning to give some of these dogs protecting inoculations as soon as they are received from the dog pound our experience is just reversed and we are able to save a large number of them. This is not only a great help as far as the experiments are concerned, but a great saving from a monetary point of view, and incidentally corroborates our previous 
results. All of these dogs are exposed to infection before being brought to the laboratory, and many of them have already contracted the disease, so that the fact that we are able to save so many speaks well for the value of the inoculations."

The experiments on the wild animals werc said to be very favourable, and apparently corroborated the work previously carried on with dogs from the dog pound. Ferry was surprised to learn that the vaccine seemed to produce marked beneficial results in other animals, and the striking results convinced him that the $B$. bronchisepticus would probably be found in other species of animals.

The bacterin is now issued in two forms: ( 1 ) As a suspension of the killed organisms in normal saline solution, and (2) in a dry state as hypodermic tablets.

Composition of Ferry's Vaccine.-It is prepared not only from several strains of $B$. bronchisepticus (6 parts), but with an admixture of those other organisms which are so constantly found complicating the primary infectionnamely, Staphylococcus pyogenes albus (I part), St. py. aureus (I part) and Streptococcus pyogenes (2 parts).

The suspensions of the dead organisms are diluted to a standard strength, and these so combined that the resulting mixture contains the desired number of organisms per cubic centimetre-namely, 1,000 million. The product is preserved with $0^{\circ} 2$ per cent. cresol. In the preparation of the tablets, the bacterial suspensions are dried, and the organisms then combined with a suitable tablet base, so that each tablet will contain 500 millions.

Therapentic Indications.-This bacterin* is indicated as a prophylactic and curative agent in all forms or types of canine distemper and sequelæ. Preventive treatment

* Wherever the term " bacterin " is employed, it needs to be carefully borne in mind that a distinction was made between a vaccine and a bacterin by the United States Bureau of Animal Industry, which laid down the ruling that in veterinary medicine all preparations which contain living organisms, whether attenuated or not, slaall be termed "vaccines," and all suspensions of dead organisms shall be denominated 
should be started early enough to allow the full course of injections to be completed a week or ten days before probable exposure, as in the case of dogs to be taken to a show. When used as a curative agent, the bacterin treatment should be started as early in the disease as possible, and it is strongly recommended that anti-distemper serum should be given in addition. In no case should the appropriate medicinal, dietetic, and hygienic treatment be neglected.

Directions for Use. - Either the liquid or a solution made by dissolving one or more of the tablets in sterile water is injected subcutaneously by means of a previously sterilised hypodermic syringe. Before withdrawing the bacterin from the vial, the surface of the rubber cap should be wiped over with a germicidal solution. It may prove easier to fill the syringe if the piston is withdrawn part way before puncturing the cap; if it is desired to withdraw I c.c., pull the plunger back slightly past the I c.c. mark. The air in the syringe should be forced gently into the vial, but not with so much pressure as to loosen the cap. By thus increasing the pressure in the bottle, it will be found that the syringe fills very readily, there being no partial vacuum to overcome. The puncture made by the needle is self-sealing, and if the instrument is sterile, the contents remaining in the vial will not be contaminated.

Dosage.-One tablet containing 500 million killed organisms is equivalent to 0.5 c.c. of the liquid bacterin. For the initial prophylactic dose, $\mathrm{I}, 000$ million organisms (I c.c. of liquid) is considered safe for a 2o-lb. dog. For smaller or larger dogs, the dose must be decreased or

\footnotetext{
"bacterins." Thus, Ferry's preparation will be called one or the other according as whether we think in English or American terms. Being a suspension of dead organisms, it may well be alluded to as a bacterin for the sake of convenience or distinction.
} 
increased accordingly, allowing I c.c. for each 20 lbs. of weight. It is probable, however, that this dosage is too small, and just recently Ferry has definitely recommended an increase* (see letter on p. 75). Injections may be made at three to four day intervals, increasing each dose according to the condition of the patient. Curative treatment should be pushed more gradually than the prophylactic treatment as a general rule.

A suggested schedule of dosage for a $20-1 \mathrm{~b}$. dog is as follows :

\begin{tabular}{|c|c|c|c|c|c|c|c|c|}
\hline \multicolumn{3}{|c|}{ Of Mixed Bacterin. } & \multicolumn{3}{|c|}{ As Prophylactic. } & \multicolumn{3}{|c|}{ As Curative. } \\
\hline $\begin{array}{l}\text { First dose } \\
\text { Second dose } \\
\text { Third dose }\end{array}$ & $\begin{array}{l}\ldots \\
\ldots \\
\ldots\end{array}$ & $\begin{array}{l}\cdots \\
\cdots \\
\cdots\end{array}$ & $\begin{array}{l}\text { Liquid. } \\
\text { I'o c.c. } \\
\text { I'5 c.c. } \\
2.5 \text { c.c. }\end{array}$ & $\begin{array}{l}\text { or } \\
", \\
",\end{array}$ & $\begin{array}{l}2 \\
3 \\
5\end{array}$ & $\begin{array}{l}\text { Liquid. } \\
\text { o.25 c.c. } \\
\text { o'5 c.c. } \\
\text { I'o c.c. }\end{array}$ & ", & $\begin{array}{c}\text { Tablets. } \\
\frac{1}{2} \\
\text { I } \\
2\end{array}$ \\
\hline
\end{tabular}

increasing the curative doses by $0^{\circ} 5$ c.c. or one tablet. Half the above doses should be given to small dogs and puppies, and the latter should receive further doses six months later. With each prophylactic dose of the bacterin it is advantageous to give 5 c.c. of anti-distemper serum, as this supplies immunising bodies similar to those found in the blood of a dog that has safely recovered from distemper. If it is definitely known that the dog has been exposed to infection, it is essential to give Io c.c. of the serum with each dose of bacterin.

Reaction to the Bacterin.-A certain degree of reaction may follow the injection, or a sick dog may appear to be worse and the symptoms more pronounced This reaction or "negative phase" should not cause alarm or the discontinuance of treatment; it is an indication that the animal organism is responding to the treatment. If

* An intimation has just been received from Detroit that the Canine Distemper Mixed Bacterin is being made four times the strength of that now being supplied, and the doses will be reduced to half, the net result being doses double the present strength. 
the reaction is very severe, however, it may be well not to increase the size of the dose, but to continue the last or even a lesser dose until only a moderate reaction is produced, when the ascending scale may be again resorted to.

Evidence upon the Efficacy or Otherwise of Ferry's Bacterin. -In addition to the observations of Ferry, Torrey, and Rahe quoted above, several veterinary practitioners have written expressing their appreciation of this vaccine and the encouraging results obtained with it. Mr. Stansfield, of Rawtenstall, the well-known breeder of collies, writes: "I have not had a single case of distemper in my kennels during I9I5 and I9I6. Several puppies I inoculated with your vaccine have been frequently exhibited, including 'Laund Laureate,' who won the Challenge Certificate at the Richmond Show, and up to now have proved immune to distemper."

My own experiences, however, have been variable, and on the whole fairly successful. Out of seven dogs which I inoculated with Ferry's bacterin at different periods, not as an experiment, but to satisfy the desires of clients. and friends, the results were as follows :

CASE I.-Collie puppy, $2 \frac{1}{2}$ months old. Gave three inoculations at the usual prescribed intervals, and obtained slight reactions each time-i.e., malaise, inappetence, rise of temperature to $103^{\circ} \mathrm{F}$. Was informed about four months later that the dog was sick with distemper, and after an illness of nearly three weeks it died.

CASE 2.-Collie puppy, $2 \frac{1}{2}$ months old. Same procedure as No. I. Reaction more marked. No history of subsequent naturally contracted disease.

Case 3.- Airedale puppy, 4 months old. Same procedure followed as laid down in the directions on preceding pages. Good reaction obtained with the first dose, but not with the second or third. Dog was poorly for about a week, an occasional cough being. noticed and slight diarrhœa; then became normal, and 
up to this day has not been a subject of distemper. Dog now three years old.

CASE 4.-Fox-terrier, 6 months old.

CASE 5.-Fox-terrier, 2 months old. In both these cases only slight disturbance of health was noticed, and after a suitable period of isolation they were returned to their owners. I heard nothing more of them, and presume if they had caught distemper I should have been notified.

CASE 6.-Pekingese, 3 months old. In this case the reaction was mostly confined to the seat of inoculation, as a painful swelling appeared about twelve hours after injection, but subsided without bursting before three days had elapsed. The dog was disinclined to feed during this period, but gradually regained its normal health. Some months later I was requested to attend it, and found it suffering from unmistakable distemper, with which it was seriously ill for more than four weeks, and was saved only with great difficulty.

CASE 7.- Irish terrier, about 5 months old. There was practically no disturbance of health following the injections, and I never was acquainted with any occurrence of illness in after years, although I knew the dog up to the age of quite four years.

These few observations cannot be regarded as a reliable proof of the efficacy of the bacterin, as the dogs' previous and subsequent histories were somewhat obscure; though it may be noted that in no case did any untoward result follow the inoculations, and that in Cases I and 6 no immunity was conferred. In each case the dog had not previously suffered with distemper so far as the owner could say, and although it might be unfair to presume that one or all of them had already acquired an immunity, yet the possibility of such a contingency must not be lost sight of. From these few cases I drew rather favourable deductions, and shall at least pursue the use of this prophylactic in practice on every possible occasion, as it seems to show promise 
On the other hand, it must be recorded in contradiction to the favourable views so far expressed that those practitioners with whom I have discussed the matter have mostly been sceptical and antagonistic to the use of any anti-distemper vaccines, dubbing them all as uncertain in their action, and sometimes even dangerous.

Based on the results of the experiments of these previously mentioned observers, bacterins containing $B$. bronchisepticus are now listed by several reputable commercial houses. As regards the "preventive" use of the preparation, failures may be explained on the ground that it is still practically in the experimental stage, and there seems reason to believe that improved methods of preparation, as well as a more satisfactory technique of administration, will ensure better results in the future.

Often the dogs are exposed too soon after treatment, and as their resistance has been lowered as a result of the introduction of antigen, they are more liable to infection than a normal dog under the same conditions. Perhaps in some cases the doses are too large, and in others too small; these questions can only be answered by experience. In Ferry's last letter to me he said: "As regards the efficacy of the vaccine as a prophylactic agent we have practically come to the conclusion that our dose is altogether too small, and that is the reason more favourable results have not been obtained. As pioneers in this line, perhaps we have been too conservative. We have carried on enough well-controlled experiments to know that a vaccine composed of $B$. bronchisepticus will protect against distemper, and we have had numerous reports from outside to that same effect; but we feel that the vaccine has not been doing itself justice, and that larger doses will improve it. To condemn the specificity of the organism on an unfavour- 
able showing of the vaccine may lead to serious results, and is unfair to the organism. It was years before typhoid vaccine made a proper showing, and yet the typhoid bacillus was still considered the cause of typhoid fever."

When using the vaccine for therapeutic purposes, it must be remembered that by the time the case is first brought to the attention of the veterinary surgeon the condition is that of an acute general infection, complicated with severe secondary infections, in which case vaccines would have very little chance of producing beneficial results. In order to overcome this situation, an anti-distemper serum has been prepared, which offers more hope than vaccines for these acute conditions, and which bids fair entirely to replace them in the future as a curative agent. A serum for prophylactic purposes is of little value unless used simultaneously with the vaccine. Serum may safely be given in any stage of the disease, the dose being 5 c.c. in mild cases, administered daily, and in severe cases the same amount twice daily.

In general practice it would appear to be a decidedly safer procedure always to use an antiserum in conjunction with a vaccine, particularly should the latter be prepared from living cultures. Similarly, while appreciating the greater possibility of obtaining a high degree of immunity with a living culture, for safety's sake, I deem the employment of a dead culture to be far preferable, since all risk of creating an outbreak of the disease is thereby eliminated. On the other hand, the immunity resulting from the inoculation of a dead culture is chiefly directed against the vital activity of the organisms-i.e., anti-bacterial rather than anti-toxic.

Cultures when dead produce, of course, less effect than when living, and this method may be conveniently used 
in the initial stages of active immunisation-to be afterwards followed by injections of the living cultures. Ferry's vaccine should prove superior to others, if for no other reason than that it is polyvalent and polymicrobial, for the cocci contained may be expected to play an important rôle in protecting against the secondary complications with which they are so frequently proved to be associated.

The whole question of preventive inoculation is so highly technical, and our present knowledge of it so uncertain-as applied to canine distemper-that I cannot close this section without deploring the fact that laymen and others insufficiently trained have access to these bacteriological products, and find it possible to experiment with animals unhindered; for without an inside knowledge of the work their conclusions can only mislead and further bewilder us. The trained pathologist and experienced practitioner are the only individuals who can adequately tackle the question with any hope of elucidating points about which we are still in the dark.

5. With Attenuated Living Cultures-Chauveau's Vaccine. -At a meeting of the Académie des Sciences (reported in Veterinary Journal, Igor), M. Chauveau intimated that experiments had been made in his institute with the object of inoculating against distemper. He had, he said, by means of intravenous injection of microbes taken from the blood and organs of a guinea-pig which had died of acute septicæmia, produced a disease in dogs which was very like distemper. Believing he had obtained, if not the specific, at least a nearly related microbe, he had inoculated a lot of dogs against this microbe; further, he had found in dogs suffering from distemper the same microbe with all its morphological and biological qualities. He succeeded in producing immunity in the dog by subcutaneous injection of cul- 
tures. His patients resisted the natural as well as the experimental infection.

Phisalix Vaccine-About six months subsequent to the communication of Copeman's paper to the Royal Society, Dr. Phisalix announced to the Société de Médecine Vétérinaire Pratique in Paris, that he had isolated the specific microbe of distemper, and that by inoculating with it in cultures of increasing virulence he had succeeded in rendering dogs immune against the disease. As regards the isolation of the specific microbe, however, he acknowledged his anticipation by M. Lignières, stating his belief that the ovoid bacterium discovered by Lignières, and named by him Pasteurella canina, was identical with the cocco-bacillus which he believed to be the causative agent of distemper. According to Dr. Phisalix, Professor Lignières contented himself with the isolation of the micro-organism, and the demonstration of its capability, when injected in pure culture, of reproducing in the dog all the classical features of distemper, the results obtained varying in accordance with the virulence of the culture, the dose employed, and the manner of inoculation-whether subcutaneous, intravenous, or intraperitoneal; but he did not apparently carry out any investigations as to the possibility of employing a culture of the micro-organism, living or dead, or its products, or both combined, for the purpose of protecting dogs against subsequent invasion of the natural disease. This, however, Dr. Phisalix claimed to have done, and he put on record certain statistics showing the results of the vaccinations he carried out.

Cultures were grown on an artificial medium of glycerinated broth, which were purposely attenuated by subcultivation or by age, and the potency of the vaccines obtained varied according to the degree of attenuation arrived at. Phisalix advocated using two vaccines differ- 
ing in strength, the first dose being 2 c.c. of a weak vaccine (for a two months old puppy) injected subcutaneously in the thigh; this to be followed in about ten days by the second or stronger vaccine injected into the opposite thigh.

Evidence For or Against the Efficacy of Phisalix Vaccine. - He showed that of 298 dogs which had been vaccinated, thirteen died. In two packs of hounds aggregating I 20 animals, in which prior to vaccination the mortality had been computed at 50 per cent, he stated that no case of distemper occurred after his prophylactic treatment.

Mr. Gray, M.R.C.V.S., said: "As far as my testing goes with Dr. Phisalix's anti-distemper vaccine-which was carried out at the suggestion of Mr. William Hunting, F.R.C.V.S.-I am satisfied that when properly used, according to the directions of Dr. Phisalix, it has great prophylactic properties." At a meeting of the Central Veterinary Society in 1905, Mr. Livesey, M.R.C.V.S., in discussing Gray's advocacy of Phisalix vaccine, was exceedingly sceptical as to its efficacy, and would not believe that the Pasteurella was the cause of distemper. This practitioner has apparently not had cause to modify his views to this day, for he still emphatically pronounces against all vaccines as yet introduced for the suppression of distemper. At the same meeting Mr. Perryman, M.R.C.V.S., expressed himself as in disagreement with the general ideas of the success of Phisalix vaccine, and still thought a field was open for experimenters to find a vaccine for the immunisation of dogs against distemper.

In the Veterinary Record for January, I905, Mr. A. Spicer, F.R.C.V.S., related having inoculated eleven dogs with Phisalix vaccine and obtaining good reactions with each of two inoculations. Three of the dogs, after some months' interval, developed severe attacks of dis- 
temper. Unfortunately no mention was made of the remaining eight, or whether any deaths occurred.

Mr. A. Sewell, M.R.C.V.S., declared that injections of the vaccine of Dr. Phisalix gave in his hands no reaction in any instance, and did not afford any protection against distemper when the inoculated animals were afterwards brought into contact with a dog suffering from the disease. He gave details of fourteen cases, in some of which the vaccine was applied three times, but in practically every instance in which the history was followed up the dogs when placed in contact caught the disease, and a large percentage of them died. Nor did any appear to suffer less severely than one would have expected had they never been vaccinated.

Finally, Professor Hobday cites seven cases in which, despite the most careful precautions to boil the syringe and thoroughly cleanse and asepticise the skin before. vaccinating with the Phisalix preparation, he was unable to obtain any reaction beyond a slight and very temporary swelling where the fluid was injected, and that dispersed within a few hours. No details were given of five of the dogs, as their histories were very uncertain, and although they had not been reported as having developed distemper no evidence was forthcoming of any contact with contagion after return to their owners. The remaining two puppies both contracted the malady two months after inoculation, and one never recovered.

Report of Committee on Phisalix Vaccine. - In consequence of the very contradictory statements of authorities on the efficacy of Phisalix vaccine, which led to public challenges, and finally to the formation of a committee of veterinary surgeons in 1903 to test its merits carefully, their conclusions, after about nine months of experimentation, were: 
"The committee consider the first experiment an entire failure, and the second inconclusive but suggestive. On the other hand, they regard the results of the third experiment as unimpeachable evidence that the vaccination failed to confer any immunity against distemper. . . . The net result of the experiment was that three of the four vaccinated pups died of distemper, while only two deaths occurred among the four unvaccinated pups."

In face of all this adverse evidence, one must reluctantly conclude that the material is valueless as a preventive.

Lignières' Polyvalent Vaccine.-In an article on "Vaccination against Distemper" printed in the Recueil de Médecine Vétérinaire for July, 1903, Professor Lignières detailed the preparation of his polyvalent vaccine. The Pasteurella canis is used, and a mixture of its various strains is grown on agar-agar, but in order safely to diminish their virulence several hundred subcultures are carried out. As with the Phisalix preparation, two vaccines are employed, the first being subjected to a temperature of $42^{\circ} \mathrm{C}$. for five days, while the second and stronger one is subjected to a like temperature for only two days. The dose of either is I c.c. No better results, however, appear to have been obtained with this vaccine than with its predecessor, and it has not yet succeeded in establishing itself as a recognised prophylactic. 


\section{CHAPTER VIII}

\section{SYMPTOMS}

Distemper is such a variable disease that it seems almost impossible to describe any one symptom which would specifically characterise it; thus, only when several of the classical phenomena are present in combination can the clinician pronounce with any genuine conviction that the animal is suffering from distemper.

From the history of the case, age of the dog, and general appearances, however, he would no doubt formulate a very shrewd idea of the animal's complaint -even in the early stages-and would probably be perfectly justified and correct in diagnosing distemper.

There are two conditions arising during or after an attack of distemper which, although they frequently do not manifest themselves, are very diagnostic of it when present, and these are the cutaneous eruption and chorea. Distemper is often insidious in its inception, and may cause such slight disturbance as to pass almost or entirely unnoticed, and a dog evincing nothing worse than an occasional sneeze or slight cough would be said to have "a cold." On such occasions, however, were a diligent search made for a causal organism, most probably a pathologist would be able to demonstrate the $B$. bronchisepticus in the laryngeal or tracheal mucus.

The disease may sometimes make a sudden appearance, and pursue an acute rapid course, with death as a not infrequent termination.

The "typical" symptoms of distemper, to be enumerated in this chapter, are so described because they are so frequently observed in this disease; however, they can 
only become typical when several appear conjointly. For instance, a mere watery eye, or an ordinary cough, or perhaps diarrhœa, without the addition of one or more of the accepted pathological conditions of distemper, could each conceivably have a separate existence of its own, arising from causes widely distinct from those of the malady under consideration.

In a large number of cases the nose and eye symptoms appear some days after the cough, and even may not appear at all ; in some other cases the dog dies of convulsions within forty-eight hours, before any other serious secondary infection has had time to become manifest.

Some authors are of opinion that broncho-pneumonia is largely responsible for the inception of nervous disorders; but it is also a fact, as just stated, that dogs may be affected with, or even succumb to, these conditions before pneumonia or any other phenomena have come into existence. We must therefore look for their cause in the formation of a toxin which attacks the central nervous system, or in a spread of infection by the causal organism of distemper from the pharynx to the brain and its coverings.

Classification of Distemper into Four Divisions.-Inasmuch as we know from clinical experience that distemper is peculiarly prone to manifest itself in some particular form in which many of the other well-known symptoms play no part, we can conveniently classify it for purposes of description into four main divisions, and place these in the order of their most usual preponderance and frequency :

I. Respiratory:

3. Nễ̃voùs.
2. Intestinal.

4. Exanthematous.

Generally, however, several organs are simultaneously affected, although the outstanding features may be those 
connected with any one of the above groups. All or any of these symptoms may be accompanied by discharge from the eyes and nose, or by either independently, and by emaciation, and sometimes these latter phenomena exist to the exclusion of most or all of the others; thus, it will be appreciated, we have to deal with a most uncertain disease.

Premonitory Symptoms.-The premonitory symptoms are: A loss of appetite; increased desire for sleep and warmth; shivering; staring coat; unusual lassitude; sometimes bad temper, and a hot, dry nose. The dog is less obedient to his master and takes less notice of him.

The animal occasionally remains bright and apparently normal, the first untoward sign noticed by the owner being a slight cough, or efforts on the part of the dog to dislodge an imaginary obstruction from its throat. Perhaps none of these initial symptoms may be observed, but a watery discharge may appear from one or both eyes, or a small bead of indurated mucus may be present at the inner corners of the eyes first thing in the morning.

The most usual train of symptoms (in the order in which they frequently occur) in a favourable case is: Inappetence, dry nose, temperature ro ${ }^{\circ} \mathrm{F}$, slight cough, nasal discharge, temperature $102^{\circ} \mathrm{F}$, constipation, increased husky cough, slight diarrhœe, temperature ${ }_{10} 2^{\circ}$ to $103^{\circ} \mathrm{F}$., dullness, shivering, increased nasal discharge, eyes gummed up in the morning, and diarrhoea more profuse. Although food is refused, there is generally an insatiable thirst, when the only consolation to the dog seems to be the sight of a dish of water. In a large number of cases this completes the picture, unless more serious complications are going to set in, when we may later find our patient in the throes of broncho-pneumonia, epilepsy, or great emaciation, with a higher temperature and increasing purulent discharges from the eyes and 
nostrils, these rapidly becoming caked about these organs, causing great mischief and inconvenience. Corneal ulcers may develop, the cough becomes incessant from extensive implication of the lungs and small bronchi, and the diarrhœa almost uncontrollable; death then may ensue from pure weakness and exhaustion, or in convulsions.

Uncomplicated Distemper.-There are no symptoms of the uncomplicated disease which can account for death, but in the sequelæ there are many. Dogs never, or rather, rarely, die from simple distemper; what carries them off is, as a rule, pneumonia or enteritis, and what renders them useless is chorea or paralysis.

The benign cases of distemper are frequently so mild in character that one is hardly aware the dog has been ailing. Even where an observant owner detects abnormality, he often ascribes the cause to worms; in fact, I feel sure that many puppies which have become subject to distemper fits-one of which has proved fatal-have been wrongly assumed to have died from intestinal parasitic affections, worms being so frequently found post-mortem.

Complicating Symptoms.-Working on the assumption that the true uncomplicated nature of distemper is merely that of a generalised catarrh of mucous membranes, the full list of complications may be cited-in the order of their usual precedence-as follows:

Bronchitis, gastritis, conjunctivitis, enteritis, pneumonia, epilepsy, keratitis, ulcerated cornea, pleurisy, peritonitis, exanthema, suppurations, otitis, ophthalmia, icterus, balanitis, nephritis, and cystitis.

Sequeloe.-As sequelæ, we see chorea, paralysis (pärtial or complete), amaurosis, cataract, leucoma, staphyloma, glaucoma, rickets, muscular atrophy, loss of hearing, scent, or voice, incontinence, and hydrocephalus. 
Temperature in Early Stages.-Before proceeding to describe the "typical" symptoms in detail, I may state that my observations regarding the temperature during the initial stages in a considerable number of cases have led me to assert definitely that it rarely exceeds $102.5^{\circ} \mathrm{F}$. I estimate the percentage temperatures to be as follows:

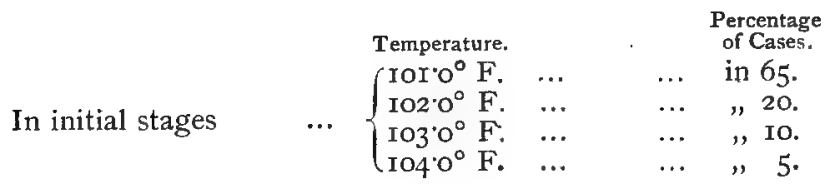

\section{Respiratory Symptoms-(I) Rhinitis.-Probably the} primary affection of the respiratory tract is catarrh of the nasal mucous membrane, attended by a discharge which is serous at the outset, but may become muco-purulent and even purulent in nature. Sneezing and shaking the head are no doubt occasioned by a nasal pruritus, which also causes the dog to rub its nose on its paws or the hearthrug or other object. From records made, I place the percentage of cases in which nasal discharge is observed in distemper at about 65 per cent.

Even as it is inconstant in appearance, it is also variable . in amount. Where there is a very copious bilateral purulent secretion, there is usually an implication of the frontal and other sinuses, in which case the discharge may be expected to remain in evidence for a considerably protracted period, and render the dog a "carrier" of infection long after convalescence.

Only rarely are these discharges hæmorrhagic in character, and then doubtless due to ulceration of the nasal septum.

(2) Laryngitis.-The larynx is implicated very early in the disease, the inflammation of which organ gives rise at first to a hoarse dry or hard cough, and later to a paroxysmal moist choking cough in which masses of 
phlegm are brought into the mouth and evacuated or swallowed; vomiting sometimes accompanies the violent efforts to dislodge this phlegm.

Gentle squeezing of the larynx from the outside will easily induce a fit of coughing; therefore, except for purposes of diagnosis, it should not be indulged in. It is not long before the catarrhal process extends down the trachea to the bronchi, and eventually to the bronchioles.

(3) Bronchitis.-Bronchitis manifests itself by a slight respiratory acceleration, perhaps (but not constantly) an increased temperature, and rhonchial sounds on auscultation. If the smail bronchi become implicated, resulting in a condition known as capillary bronchitis, there is a further frequency of respiratory movement, greater difficulty in getting breath, and an obstinacy on the part of the animal to move, such movement usually accentuating the cough and distressing the dog. Auscultation will now reveal dry or moist râles, the former in the earlier stages, due to the presence of viscid secretion in the bronchial tubes, or a thickening of their walls, and the sound produced has a whistling, musical, or squeaking quality; the moist râles are in evidence so soon as the secretion becomes liquid, and one hears crackling, crepitant, or bubbly sounds.

(4) Broncho-pneumonia.-If at this stage the patient has become, or was previously, weak and unable to expel the accumulations of bronchial secretion, a further and serious complication arises, which doubtless accounts for many deaths in distemper. Owing to the bronchioles becoming clogged with muco-purulent exudate, consolidated patches in single lobes are formed, which may extend to larger areas. This condition is known as catarrhal or broncho-pneumonia. Its onset is marked usually by a considerable rise in temperature, great dyspnœa, rapidity of pulse and respirations, feeble but 
persistent and distressing cough, a diminution or disappearance of respiratory râles, and sometimes at the last by a stinking nasal discharge. When severe, the dog seems to find a difficulty in breathing except in an upright position, and he blows out his cheeks at each expiration-always an ominous sign.

Only too frequently in such cases death supervenes, and is ushered in by a rapid, feeble pulse, diminished temperature (perhaps even to subnormal), cold extremities, short, decreasing respiration, œdema of the lungs (with severe dyspnœa and tympany on percussion), and cyanosis, the patient becoming delirious or comatose, and succumbing finally to asphyxia. I treated recently for several weeks a case of this sort in a two-year-old Alsatian wolfhound, and towards the termination of its illness it showed all the distressing symptoms herein enumerated, in addition to excessive weakness, emaciation, and restlessness. Its temperature, however, hovered about $103^{\circ} \mathrm{F}$, and never once exceeded $104^{\circ} \mathrm{F}$.; but its peculiar retching kind of cough, accompanied by cries as of pain, was most pathetic to witness. Upon postmortem examination, I was rather surprised to find that only about one-third of the lung appeared to be implicated.

(5) Croupous Pneumonia.-Distemper subjects have been occasionally observed to suffer from croupous pneumonia, occurring sometimes as a terminal pneumonia, and hastening a fatal termination to the primary disease. It is marked by inflammation of one or more lobes of the lung, usually beginning in the lower third, the organ being first intensely congested or engorged, afterwards becoming red and consolidated from accumulation of exudate and blood-cells in the alveoli (red hepatisation); and, later, grey from degeneration of the exudates (grey hepatisation), which are finally absorbed. 
It is attended with the usual shivering, inappetence, dullness, and a sudden elevation of temperature. There is also difficult and rapid breathing and hacking cough, and, when complicated by pleurisy, the respiratory movements are shallow, and the dog evinces pain if picked up. In favourable cases the pulse remains fairly full, and the temperature after the crisis falls progressively to normal, resolution occurring in about eight or nine days. If a fatal termination is to ensue, the pulse becomes thin and

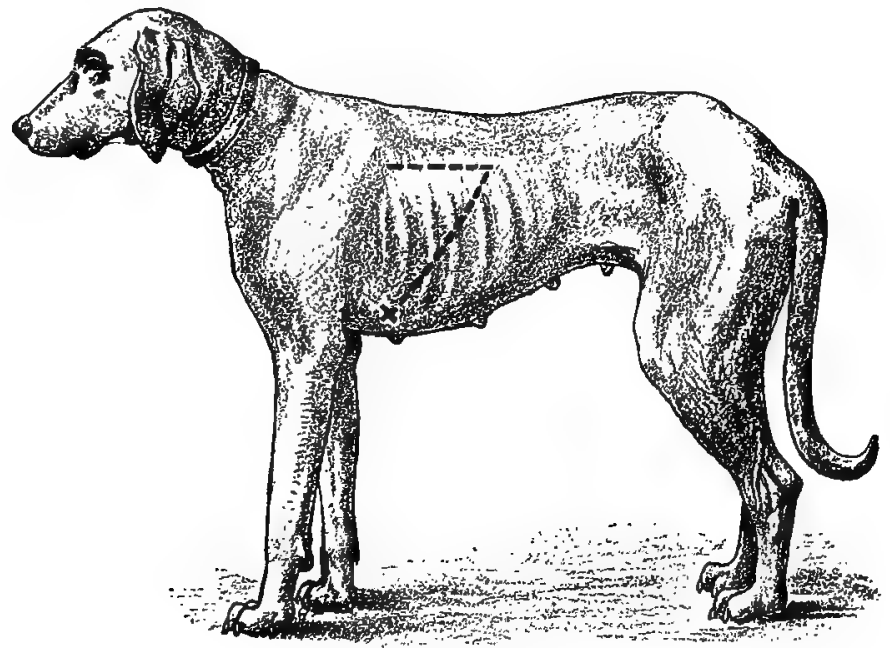

Fig. 6.-Field of Percussion.

$X$, Location of Heart-beat.

(Müller and Glass, "Diseases of the Dog.")

irregular, dyspnœa is excessive, with a blowing respiration, great restlessness, perhaps blood-stained expectoration, ending in either coma, convulsions, or delirium.

Percussion over the normal areas of lung will give the tympanitic sounds, whilst over the congested or consolidated areas dullness will be in evidence, and the diseased parts can usually be sharply defined. Fine dry crepitations may be heard at inspiration during the period of 
engorgement; they gradually diminish as the lung becomes hepatised, but their renewal at a later date will signify that recovery is taking place.

(6) Pleurisy.-Pleurisy in my experience occurs but rarely in cases of distemper, and then is always a serious complication. In the acute type the pleura first becomes reddened, and a soft grey lymph exudes. This is the dry stage, signified by impaired chest movement, sounds of friction on auscultation, and pain in the sides of the chest. The disease may stop here, or may progress to the second stage, in which a copious exudation of serum occurs (stage of liquid effusion), bringing in its wake increased dyspnœe, but a diminution of pain. There is now dullness on percussion over the fluid, the area of dullness changing with changes of position; the intercostal depressions tend to become effaced owing to the internal pressure of the thoracic liquid.

No sounds appear to come through the liquid, but above its level an increased resonance and friction sounds may be heard. Frequently during the second stage the dog succumbs from exhaustion. I recently treated a Yorkshire terrier puppy which showed no other symptoms during the first eight days than those of pleurisy, and I considered I was dealing solely with that disease, until, on the ninth day, three of the characteristic symptoms of distemper set in.

(7) Gangrene of the Lungs.-On very rare occasions gangrene of the lungs has been known to supervene. It can be detected by the very foul odour of the breath combined with a putrid-smelling nasal discharge; when present it is always fatal.

(8) Edema of the Lungs.-This condition is observed in very young, very old, or weak, debilitated animals, and is a very grave symptom indeed. It follows in the wake, not only of distemper, but of any very debilitating 
disease which weakens the left side of the heart and renders it unable to force the venous blood through the lungs.

Upon its development breathing becomes extremely difficult and stertorous, and an opaque blood-stained fluid escapes from the nostrils, particularly just before and after death. Auscultation reveals loud rattling murmurs throughout the lung, and the pulse is irregular.

Proportionate Frequency of Various Lung Lesions.-It is reasonably safe to assert that the majority of dogs suffering from distemper do not as a rule contract lobar pneumonia, pleurisy, œdema or gangrene of the lungs. According to Ferry and his co-workers, records taken of sixty-eight cases revealed the lung lesions in the following proportions:

\begin{tabular}{llll} 
Broncho-pneumonia & $\ldots$ & 45 (mostly only slightly affected). \\
\multicolumn{2}{l}{ Croupous pneumonia } & $\ldots$ & I 3 \\
CEdema ... & $\ldots$ & $\ldots$ & I \\
No lung lesions & $\ldots$ & $\ldots$ & 9
\end{tabular}

A goodly proportion of patients, however, never appear to suffer from anything worse than laryngitis or bronchitis, especially as judged by their high spirits and steady appetites during these mild attacks, and in this connection I might aptly quote M'Gowan, who declares: "What has chiefly surprised me in cases of congestion of the lungs, pneumonia, and empyemata, is the extent of the morbid changes in the lung when contrasted with the slight symptoms they produce in the animal during life. I have frequently seen a fat, well-nourished puppy running about, playing actively, and taking its food well; but on performing a postmortem a day or two later, have found the lungs practically solid with a purulent pneumonia, which must have lasted some time. I have seen nine cases of such pneumonia and two cases of empyema in the dog due to 
a pure infection with the distemper organism. Here, again, in the case of empyema, the symptoms exhibited during life did not lead one to expect, as was found, the pleural cavities to be filled with pus, and the lung almost completely solidified or collapsed."

It has been asserted, and no doubt correctly, by many authorities whose opinions must command the utmost respect, that pneumonia is a complication of distemper ; but in consequence of its being so frequently in association with distemper I regard it as being a very confirmatory and diagnostic symptom, even as are also the abdominal and other conditions.

Nevertheless, individually none of them is characteristic, though two or three in combination should be sufficient evidence to expel all further doubts.

Uncomplicated Distemper.-Uncomplicated distemper is thought to be a pure and simple catarrh of the respiratory mucous membranes-in fact, coryza-marked by a slight rise in temperature $\left(\mathrm{I}^{\circ} \mathrm{F}\right.$ ), watery discharge from eyes and nose, and a husky cough. These three affections are found together in about 55 per cent. of cases, whilst cough may be observed in quite 90 per cent., which seems to prove that the primary infection is in the trachea and bronchi, and not in the nose.

Eye Symptoms.-Ocular lesions may be conveniently described here, since their production is frequently brought about by the direct spread of infection from the nose via the nasal duct. When the secretions from the nose have become purulent, this duct is more or less occluded, with the result that the exit for tears is blocked, and it is possible that infection may rapidly spread along this channel.

Conjunctivitis. - In a number of cases nothing more formidable than a watery effusion from the eyes is observed, accompanied by a great distaste for strong 
light on the part of the dog, especially in small breeds such as Pekingese, Pomeranians, etc., though usually we find it supplemented by a serous or purulent conjunctivitis. In such cases photophobia is almost constantly present, corresponding in degree to the severity of the inflammation.

In very many cases this affection is not manifest until about a week has elapsed after the first noticeable signs of sickness, though in fewer instances it occurs much earlier or may not, in fact, occur at all. The ocular catarrhal discharge is generally characteristic of distemper, and may be found roughly in about 60 per cent. of cases in combination with other symptoms, but very rarely alone. In forty-eight hours this serous or mucopurulent effusion may, in graver cases, have developed into a thick yellow purulent exudate (blenorrhœa) which adheres obstinately to the eyelids and lashes, where it dries into crusts, effectually gumming the lids together and imprisoning the succeeding formations of pus in the orbit.

Simultaneously a thick purulent discharge is exuding from the nostrils, around which it clings and dries, causing the nose to become cracked and ulcerated. In consequence of the considerable amount of mechanical obstruction often caused to breathing, the discharges are violently sneezed out.

Ulcerated Cornea.-The condition advances from bad to worse, for not only does the dog injure its eyes by scratching and rubbing at them in its futile endeavours to remove the offending concretions, but, as might be expected, the accumulations of stale pus set up excoriations of the skin surrounding the eye, attended by loss of hair, and graver still, often cause ulceration of the cornea. When these excretions are cleaned away and the eye opened, the conjunctival mucous membrane is found 
extremely congested and swollen, and photophobia is intense; the eye at this stage is very frequently affected with a vascular keratitis, and in some instances becomes further complicated with panophthalmia.

The ulcerated condition of the cornea is a fairly common occurrence in distemper, the lesion being located usually in the centre as a small shallow depression, varying in size from that of a pin's point to $\frac{3}{16}$ inch. It may have a traumatic origin-inflicted by the patient itself-or may have been preceded by small vesicles which later burst, leaving the cornea thinned at this spot. We know, however, from clinical experience, that corneal ulcers sometimes occur in the absence of any of these exciting causes; thus we should not be dogmatic in expressing an opinion. If the ulcers are neglected, one not infrequently finds that incomplete perforation takes place, with a resulting hernia of Descemet's membrane.

Staphyloma.-As a further complication, this membrane may now become pervious and a quantity of the aqueous fluid be lost through the aperture, causing in its turn a prolapse of the iris. This condition is termed staphyloma.

It is a debatable point as to whether sight is ever regained after this grave condition, and I believe the most favourable termination would be the retention of only a very blurred vision. Whilst an uncomplicated ulcer usually clears up quite well, leaving no trace of its previous existence, a staphyloma is often the forerunner of a permanent disfigurement in the shape of cataract of the lens, or irreducible corneal opacity with partial or total loss of sight, or panophthalmitis with probable total extirpation of the eyeball.

Panophthalmitis.-Panophthalmitis is an inflammation of all the tissues or structures of the eyeball, and is a very grave complication indeed. Intense pain is produced and the eyelids are constantly closed. Accumula- 
tions of pus are soon found in the anterior chamber, which find exit through the corneal perforation, and the aqueous and vitreous humours may escape by the same channel. All sight has then vanished; the eyeball collapses and shrivels, and there is no alternative but to totally remove what remains by surgical means. In rare cases panophthalmia may suddenly appear in the absence of any septic infection through a corneal perforation, and run an acute rapid destructive course in which all parts of the eye are intensely inflamed, and rupture of the cornea accomplished from within, with consequent loss of the humours. Panophthalmia may be transferred from an affected to an unaffected eye by a sympathetic process, the condition being known as "sympathetic panophthalmitis."

Parenchymatous Keratitis. - Not rarely, instead of ulcerative processes occurring one finds a parenchymatous keratitis or bluish milky opacity diffused over the whole of the anterior part of the eyeball (which has been described by some as having a "ground-glass" appearance) and which may subsequently extend inwards and involve the iris, with resulting iritis. This last condition is marked by pain, congestion in the ciliary region, photophobia, contraction of the pupil and discoloration of the iris. These diffuse opacities sometimes remain for the rest of the animal's life, especially in old dogs, and whilst they do not seem absolutely to deprive the eye of all sight for near objects, they certainly seriously curtail vision for long distances.

Interstitial keratitis, parenchymatous keratitis, as also ulcerative keratitis, may affect one or both eyes, panophthalmia usually only one eye, and ordinary benign conjunctivitis practically always both eyes. Opacitieslike many other pathological conditions-though arising sometimes in a few hours, frequently require many 
weeks of treatment before dispersal is effected, and may even be permanent.

The sequeloe which may arise from lesions of the eye in distemper are :

(I) Lencoma.-Cicatricial opacities of the cornea, following ulcerative keratitis, may vary in size and density, and remain sometimes permanently. Occasion-

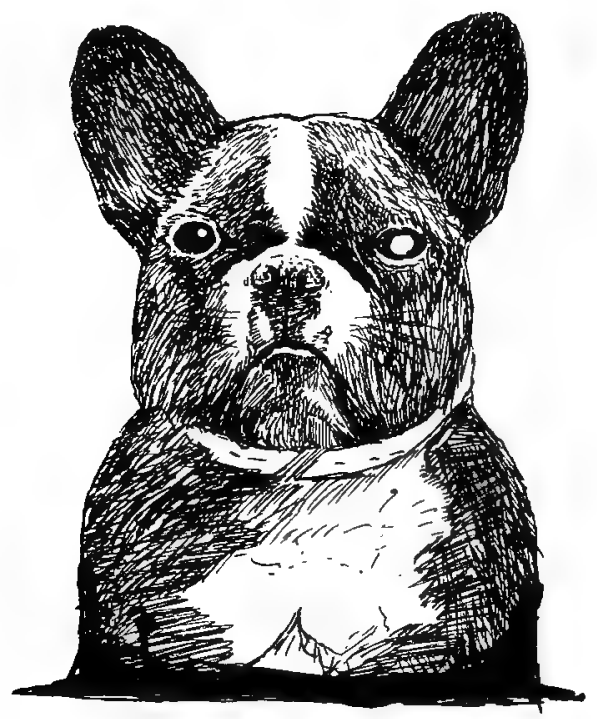

Fig. 7.-Leucoma of the Left Eye.

ally the cornea undergoes pigmentation, "pigmentum nigrum " being the dark colouring matter which appears in spots on the internal surface of the corneal membrane after ulceration.

(2) Cataract.-An opacity of the crystalline lens or of its capsule. Grey cataract may occur as a result of disease of other parts of the eye, which has forced the lens into abnormal positions, or rendered its nutrient supply defective. The discoloration or opacity may be 
in localised spots or diffused, and where difficulty is experienced in making a thorough examination of the lens for diagnostic purposes the best plan is to dilate the pupil by.means of atropine. Prognosis is unfavour-

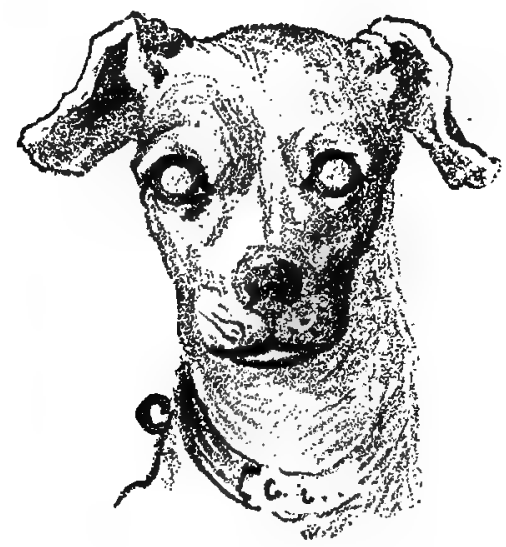

Fig. 8.-Grey Cataract of Both Eyes. (Müller and Glass, "Diseases of the Dog.")

able: the sight is impeded or entirely lost according to the extent of the lesion.

(3) Amaurosis, or complete blindness, occurs sometimes in the absence of any apparent lesions of the eye, but probably is a sequel to retinitis, atrophy of the optic nerve, or diseases of the brain. It is known also as "Black Cataract," presumably from the absence of white or other visible lesions. No pain is connected with the condition, but prognosis is very grave, as only in the rarest instances have dogs been known to recover their sight after the onset of amaurosis.

(4) Glaucoma, or dropsy of the anterior chamber; a secondary disease marked by intense intra-ocular pressure, and resulting in hardness of the eye, atrophy of the optic disc, and blindness. The cornea is rendered opaque and the conjunctivæ frequently inflamed. 
(5) Total Excision.-A grave termination which becomes imperative in some cases of panophthalmitis and glaucoma.

(6) Nystagmus. - This name has been given to a continuous rolling movement of the eye, and is said to have been observed as a rare sequel to distemper, but in fourteen years' experience I have never encountered a case in the lower animals (except in connection with concussion of the brain after an accident), though it is occasionally seen in the human being. Fortunately all these sequelæ are quite infrequent, the great majority of cases of distemper leaving in their wake no serious complication connected with the eye.

At the moment I am treating a Pekingese spaniel nine months old, and said to be "over distemper." It was brought to me for examination and opinion one Saturday morning. It was lively and feeding well, but had a watery discharge from both eyes, though more particularly from the near side one, and a snuffling of the nose; temperature was $100^{\circ} 6^{\circ} \mathrm{F}$., and the owner had noted a state of constipation. On the left flank was a minute pimple in the centre of what appeared to be a small patch of dry eczema. Notwithstanding the history, I diagnosed distemper. The following Monday it was brought again, and I was surprised to find the left eye quite opaque and a well-developed corneal ulcer present attended by profuse lachrymation and moderate photophobia. Con. stipation still in evidence, also sneezing; temperature ${ }^{\circ}{ }^{\circ} \mathrm{o}^{\circ} \mathrm{F}$. and appetite good. Suitable treatment was prescribed, and by the end of the week much improvement had taken place in the eye and in the general condition of the dog. I am, however, making no hasty prognosis, although the case is doing wonderfully well. This is a good example of how quickly corneal ulcers and keratitis will appear even in the absence of any purulent discharges, and how early they may appear in the disease.

II. Gastro-Intestinal Symptoms. - There are many symptoms associated with the "abdominal" or "digestive" type of the disease, foremost of which is probably loss of appetite; although many cases are to be met in which the hunger and sprightly activities of the dog are in no wise abated-even throughout the course of a mild attack. 
Oral Lesions.-Many writers have spoken of the socalled oral lesions of distemper, in which ulceration of the buccal mucous membrane has played a prominent part. Personally I have only observed these lesions in a small minority of cases. More frequently I have noticed a hyperæmic appearance of the gums, which have bled upon the slightest provocation. The teeth also at times show a dirty brown discoloration, and the tongue may be furred. Some authorities have included necrosis and gangrene of the free anterior portion of the tongue as a lesion of distemper; but in my opinion they are confounding the symptoms of this malady with those of Stuttgart disease.

Occasionally a vesicular eruption may be detected about the lips and at the angles of the mouth, which, however, may be part of the exanthema sometimes found in distemper on other parts of the body, particularly the abdomen and thighs.

Pharyngitis.-As a result of the catarrhal affection, the pharynx and larynx may be swollen and slightly inflamed at the onset, giving rise to sore throat, cough, and profuse expectoration, whilst difficulties in swallowing or breathing may also become apparent. In such a contingency, if medicinal treatment is contemplated, it is a wise precaution to ascertain first whether the dog will be able to receive the medicine safely and swallow it.

Vomiting.-Vomiting a thin, frothy mucus is frequently one of the earliest symptoms, indicating irritation of the stomach in consequence of a catarrh of that organ, which may vary in its intensity as in its incidence. Too much importance need not, however, be attached at any time to vomition in the dog, as he is able to accomplish this act at will, and frequently does so. Being forced to submit only too often to ignorance in his treatment, it is perhaps fortunate for him that he possesses this 
power. There is generally increased thirst, a dry, hot mouth, and some degree of constipation.

Diarrhea.-A considerable conflict of opinion exists as to whether diarrhœa is an early symptom, or appears at a later stage in the course of the malady. From my own observations, it appears usually after a brief period of costiveness, though sometimes only at a very late stage, and at other times precedes every other discernible symptom. Ferry declares: "An important point is the fact that diarrhoea is one of the earliest symptoms in distemper, and it is found in nearly every case providing we look for it early enough. I have often seen it before I have recorded a cough; I suppose because it is a symptom that leaves behind a record of its own. I soon learned that if I saw a young dog with a watery diarrhœa I would be pretty sure to find a case of distemper within a few days."

Infection in the Foces.-Ferry frequently found his $B$. bronchisepticus in the fæces of distempered dogs, and maintained that diarrhœa was thus to be regarded as a classical symptom, and, being one of the earliest, of the gravest importance. In contention with this view, M'Gowan says: "The group of symptoms usually classed as abdominal distemper to my mind are not indicative of distemper per se, but of a cachectic septic condition entirely secondary to the disease. It includes wasting, ulcers on mucous membrane of mouth and gums, dirty teeth, stinking breath, vomiting, and diarrhœe. Usually along with these are included jaundice, and, in some cases, bloody diarrhoa and the passage of pure blood per rectum."

It is difficult to reconcile these contrary opinions, but greater credence seems due to Ferry from the obvious clinical fact that diarrhœa is so frequently (though not in the majority of cases) seen before any other pheno- 
menon, and secondary infections can hardly be believed to manifest themselves in anticipation of primary ones. It is seldom that any case of distemper ever runs its full course without the incidence of diarrhea at some period, and, on the whole, we usually expect it early.

Sometimes what appears to be only a case of simple but obstinate diarrhœa, enduring over a week or two, suddenly terminates by the advent of nervous symptoms and, it may be, death in convulsions.

Constipation.-Where constipation is a prelude to a generalised attack, it may be suggested in explanation that since the dog has no appetite, no food has been consumed, and therefore none can be passed; or that the absence of food in the alimentary canal has brought about a cessation of peristalsis, or a condition of intestinal stasis; further, since the presence of food in the mouth and stomach is necessary to stimulate secretion of digestive juices, absence would account for non-secretion and consequent dry condition of the mucous membrane of the bowel. This condition is then overcome by the action of the invading organisms, which set up a general gastro-intestinal catarrh, with resulting diarrhœea. If no formed fæces exist, fluid slimy or frothy stools containing mucus, which soon become very fotid, are then evacuated.

Gastro-Enteritis.-Very often the catarrh is so intense that it merges into an acute inflammation, accompanied by colic, tenesmus, and the passage of sanguineous evacuations or even of pure blood. If an autopsy were made at this stage the intestinal tract would be found full of mucus-evidence of gastro-intestinal catarrh-and the mucous membrane observed to be very swollen and corrugated, the convexities of the ridges being hæmorrhagic, or even ulcerous, and presenting an appearance similar to that so often observed in swine fever, with the excep- 
tion that the ulcers of swine fever are very typical of that disease.

The condition may be aggravated by the presence of worms, improper feeding, or the administration of quack nostrums, many of which are totally contra-indicatedparticularly severe purgatives and vermifuges.

Tenesmus and Rectal Prolapse.-Tenesmus or straining becomes persistent and painful, in consequence of which,

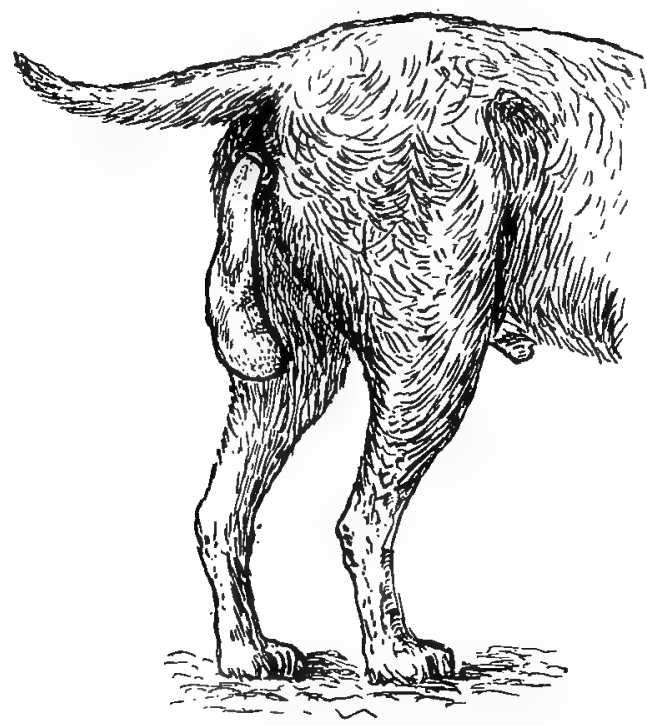

Fig. 9.-Prolapse of the Rectum.

not uncommonly, the veterinary surgeon has to contend with a grave complication in the prolapse of the rectum.

Protracted diarrhœa and tenesmus will set up a rapid emaciation, and the animal will evince signs of internal pain, such as arching of the back, general uneasiness, tenderness on pressure of the abdomen-which is tense and contracted-and excessive thirst. If, then, we observe the passage of pure blood per anum, this may be evidence either of ulceration or of a probable intussus- 
ception, which frequently leads to a fatal termination. Diarrhœa in itself, provided it proves amenable to treatment and is not reducing the dog to a state of great weakness, need not be feared as a very serious sign; in fact, should an obstinate diarrhœa suddenly cease without apparent cause, it is viewed with some concern, as it is thought that the retention of poisons which would otherwise have been eliminated sets up a toxæmia which attacks the central nervous system, producing nervous manifestations such as chorea, convulsions, etc.

Emaciation.-Emaciation is peculiarly typical of distemper, and has been observed by practically every writer on the subject. Rapid emaciation, with or without a very marked diarrhœa, is a symptom not to be overlooked. The dog may lie about in a listless manner for a week or two, losing flesh rapidly, with no marked temperature, or possibly with a subnormal one; sometimes with a cough, now and then so slight as to be entirely overlooked, when suddenly it will develop a purulent discharge from the eyes and nose and die within a few days from a most virulent type of the disease. In such cases, even though of such short duration, the loss of flesh will often be remarkable. It can be noticed again and again, even in mild and favourable attacks, what a comparatively great loss of weight takes place, and severe symptoms do not appear to be essential to its incidence.

After the discharges have made their appearance in the virulent and rapid types of distemper, the chances of recovery with any kind of treatment are practically nil. Progressive wasting may be produced or encouraged by excessive purgation, and is certainly always the sequel to a long-continued debilitating diarrhœa.

The putrid type of distemper often occurs as a complication of what had previously been a benign attack, and in which we find the development of a marked debility, 
speedy emaciation, profuse, stinking, and sometimes sanguineous, nasal discharge-indicating extensive ulceration of the nasal mucous membranes, or pus in the sinuses-an irritant and very excoriating ocular discharge, with, on rare occasions, an exudation from the ear; the fæcal material is a mixture of blood, mucus, and offensive bile, the body of the poor wretched beast emitting a vile smell characteristic of distemper; and after a miserable existence of varied duration, finally succumbing to the inevitable.

Jaundice. - Under the heading of gastro-intestinal lesions one might very conveniently consider the hepatic symptoms which sometimes arise with or after distemper, since they are closely related to those of the alimentary canal. Jaundice is a somewhat rare, though very frequently fatal, complication of distemper, in which the bile invades every tissue of the body, via the blood stream, causing a characteristic yellow hue to skin and visible mucous membranes. These yellow tints set in at a somewhat late stage of the complaint, the sclerotic and conjunctiva then being first invaded, followed in turn by the mucous membranes, skin, and urine. The latter takes on a deeper hue, and the fæces are variously black, greenish, or clay-coloured and very offensive. The breath is bad, vomition is sometimes excessive, and the vomit often mixed with blood, the animal very depressed and constipated. Sometimes, however, there is diarrhœa, the excrements being bloody; or pure blood may at times be voided. From the moment the yellow tinge sets in, the fæces become greyish-white, owing to the stoppage of the flow of bile into the intestines, and they contain much undigested fat. The antiseptic effect of the bile is absent, which fact-coupled with the presence of undigested fat in the frees-makes the latter very fœid and unpleasant. 
There is pain on pressure over the region of the liver, with occasional enlargement and hardness of that organ. The pulse becomes weaker, and the animal more feeble, the extremities cold, and emaciation pronounced, whilst convulsions succeeded by profound coma usually precede death.

Cause.-The duodenum as well as the rest of the intestine is filled with a pale stringy mucus (absence of bile), and the opening of the common duct is occluded by spread of the intestinal inflammation to its mucous membrane, obstructive jaundice being thus produced. Swelling of intestinal mucous membrane adjacent to the opening of the bile duct is another frequent cause of obstruction in cases of distemper.

Youatt found the condition most frequently in hounds and greyhounds, particularly in cases showing little catarrhal exudation from the nose. I fail to see a connection, however, between the two phenomena. The common experience seems to be that when jaundice appears as a complication, it does so in particular outbreaks, or during certain years or seasons. The few cases I have seen of the combination, however, have been quite sporadic, and apparently independent of either time or place, nor have they been confined to any particular breed. Age has no influence in its production, young and old being equally susceptible.

While it is my opinion that the jaundice of distemper is a catarrhal jaundice due to duodenal catarrh, there are others who consider it is of partly hæmatogenous and partly hepatogenous origin, which latter includes toxæmic jaundice and the jaundice seen in malaria, typhoid, typhus, and yellow atrophy of the liver.

Weber, of Paris, remarks: "It prevails among dogs which are left in the charge of keepers, and, as often happens, are subjected to strong drastic purgatives, or 
to emetics, with a view to cure the distemper." Other predisposing causes are chills, fatigue, and obstinate constipation.

There is usually little to be found on post-mortem examination which would seem to account adequately for death; thus, one can only conclude that an intoxication by some poison, as evidenced by prostration, insensibility, and subnormal temperature, is the direct cause of a fatal termination.

If a favourable turn should, however, occur, it will be evinced first by diminished discoloration of the urine, then by darkening of the fæcal tint, more regular and better pulse, increase of temperature, greater vivacity, and a healthier hue of the visible mucous membranes.

III. Nervous Symptoms.-There are no symptoms of this dread disease which are more fraught with mischief or mortality, or less amenable to treatment, than the many nervous complications unfortunately so frequent in distemper.

They are variously observed to precede all other symptoms, to occur during the course of the malady, or as a termination. Even some time after supposed complete recovery, cases have been recorded in which nervous sequelæ have arisen.

Etiology.-Their etiology seems to remain more or less obscure, although several theories have been advanced. For instance, Youatt considered that an inflammation of the eye or of the nose might, from very proximity, soon be communicated to the brain, probably via the cribriform plates. Blain, however, says: "It is not unlikely that in some few cases a true metastasis (transference of disease from one organ to another) occurs, for we occasionally have a very sudden attack of epilepsy without any of those premonitory symptoms which usually predict with malign certainty the dis- 
temper fits; and there is more reason to conclude this since a single fit, not followed up by another, particularly when it appears early in the complaint, is often not injurious, and therefore we may suppose it the consequence of a sudden metastasis, which as quickly returns to its original seat."

M'Gowan (Journ. Path. and Bact., vol. xv., I9I I) intimated his opinion that "complications were due to the spread of the distemper organism to other parts . . . and chorea appeared to be due to an extension of infection from the middle ear, or roof of the nose, to the meninges, thereby causing a meningitis. I have observed three such cases, and in one a scanty growth of the distemper organism was obtained pure from the brain, and a histological examination of the brain showed the presence of a meningitis. I would place all the various paralyses that occur in distemper in the group of complications attributable to toxins. In seven cases of this kind, all of which were of the nature of paraplegia, histological examination showed small-celled infiltration of the vessels and grey matter of the cord, a condition indicating poisoning of the cord by some toxin. Cultures made from the cord were sterile."

Hutyra and Marek are of opinion that the specific distemper virus causes an early involvement of the nervous system by the development of degenerative changes in the brain and spinal cord, which, in some cases, are subsequently manifested by striking nervous symptoms.

Coma.-Cases of distemper may begin with great depression and dullness; there is a marked apathy, and sometimes even deep coma. This early listlessness and general prostration undoubtedly arise from a lack of healthy nervous energy; but the phenomena about to be described are the consequence of a morbid energy that is sometimes partial, in which case, possibly, the spinal 
cord is principally affected, or it may be total, when we conclude the brain is the seat of attack. The effects naturally vary according to the part of the central nervous system which is affected.

This condition of coma may in a great many cases be accompanied by periods of excitement, nervousness, restlessness, yelping, and even true delirium, succeeded again by marked depression. These disturbances may prove to be the only manifestations of nervous disorder.

Clonic Spasms.-We may next observe twitchings of various muscles or groups of muscles-e.g., in the lips, eyelids, cheeks, masseters, temporals, etc., followed perhaps by epileptiform convulsions. Since clonic spasms of the muscles of mastication produce an automatic opening and closing of the mouth, the saliva is whipped into foam, and this so-called "foaming at the mouth," combined with the convulsive movements of the whole body, may lead one to suppose that the animal is in a fit.

Epilepsy or Fits.-A true epileptiform seizure, however, implies loss of consciousness. Fits may occur at long intervals, or may follow so rapidly one on the other that the animal is hardly ever at rest. Sometimes a fit is preceded by general restlessness or excitement, in which the dog whimpers or yelps, and runs aimlessly about, occasionally stopping to stare stupidly about him, and then becomes suddenly convulsed, falls down, and loses consciousness; or the animal may lie quietly from the termination of one seizure to the beginning of another. Owing to the occasional relaxation of the anal and vesicular sphincters, urine and fæces may be involuntarily passed. The prognosis is extremely serious when fit after fit attacks the poor beast without intermission, lasting over a considerable period, and a fatal termination may fairly safely be predicted. 
If the dog regains consciousness, it appears such a pitiable wreck that one feels constrained to abandon hope and end its miseries. In some cases dogs become comatose immediately after cessation of convulsions, and may remain so for many hours, or may never regain consciousness. Opinions differ as to whether fits are an early symptom; my own experience is in agreement with that of Gray, who says convulsions in the early or initial stage of distemper are rare, and when met with are usually attributable to causes such as intestinal parasites, the presence of œstrum, excitement, or indigestion, etc. As a rule, these nervous phenomena are not regarded as evidences of an approaching attack of distemper, especially as symptoms suggestive of the disease, such as catarrh of the eyes and nostrils, may not appear until from one to three weeks afterwards.

On the other hand, Ferry has stated: "A very important early symptom is 'fits.' How many a defenceless cur has been condemned to die at the point of a gun, with the diagnosis of rabies behind it, when the dumb brute was really in the throes of distemper. The dog was frothing at the mouth, of course, and its eyes were glassy, but that does not signify it was mad."

Personally I have encountered but few cases in practice in which a puppy brought to me suffering from epilepsy has later turned out to be a subject of distemper; and one may be excused for diagnosing "epilepsy of unknown origin," or "worms," where no symptom characteristic of any other condition has as yet presented itself.

Epilepsy versus Rabies. - Speaking of rabies, it is undoubtedly true that the stage of furious excitement associated with nervous distemper has often led to the premature death of the animal through a mistaken diagnosis, for the dog tears up the ground, runs at its 
attendant or owner, bites at everything within reach, and often at itself, and looks wild-eyed and mad.

These phenomena, however, usually abate quickly when distemper is the root cause, leaving the animal greatly exhausted and very depressed. In rabies they would not appear so suddenly, nor attain the great violence which so frequently characterises epilepsy. The latter never accompanies rabies in any stage of that disease.

A succession of such furious manifestations, lasting perhaps two or three days, usually effects a fatal termination, for the animal is worn down and sinks from exhaustion. Cases are not unknown in which affected dogs develop a tendency to move in a circle, walking or trotting round and round, always one way, and quite oblivious to their surroundings or the voices of those they know best. Another curious manifestation is that in which the animal, when in a recumbent position, appears to be running as fast as it can, all legs being in progressive motion, and the dog simultaneously whining or moaning. In both conditions the brain is apparently implicated, and both are always extremely ominous.

Warning of Fits.-Fits, as occurring in distemper, are usually preceded by some warning, and may possibly be averted if carefully watched for. For instance, we notice local twitchings, already referred to, as affecting the cheeks, mouth, or eyelids, restlessness, and hot head; these would be entirely absent in cases of fits arising from teething, worms, or unusual excitement, for here the animal is one moment perfectly normal and at play, and the next he is unconscious.

Youatt sums up the situation very nicely in the following paragraph: "However indisposed to eat, the dog may have previously been, the appetite returns when the fits are at hand, and the animal becomes 
absolutely voracious. Nature seems to be providing for the great expenditure of power which epilepsy will soon occasion. The mucus almost entirely disappears from the eyes, although the discharge from the nose may continue unabated; and for an hour or more before the fit there will be a champing of the lower jaw, frothing at the mouth, and discharge of saliva. The champing of the lower jaw will be seen at least twelve hours before the first fit, and will for a little while precede every other." I might add that diarrhœa also receives a temporary check, but how far this is concerned in helping to produce epilepsy I cannot say.

When epilepsy makes its appearance quite late in the disease, it is always a more sinister sign than when it is manifested early, for it seems more prone to lead to the more chronic nervous affections, such as chorea and paraplegia, and perhaps even to coma and death.

Cerebral Meningitis and its Sequeloe.-An occasional sequel to distemper is the production of meningitis, which, among other causes, may be directly due to an extension of inflammation from neighbouring structures to the meninges. In some cases symptoms occur that indicate irritation of the nerves at the base of the brainsuch as strabismus or squint, ptosis or drooping of the eyelid, optic neuritis, facial paralysis, muscular spasms, etc. In this connection it is interesting to note that ptosis and strabismus are also complications of human measles. Dogs are liable to all these affections, though they are seen but rarely.

Chorea, or St. Vitus' Dance.-More commonly they are stricken with chorea, which is apparently an interrupted or intermittent supply of nerve force to the muscles, which is attended with irritability and depression, or with mental impairment. It may become pronounced at any stage of the disease, but in the vast majority of cases 
it occurs near the crisis or during convalescence, and occasionally long afterwards. A convulsive involuntary twitching attacks sometimes the head, sometimes one or more limbs, and at other times the whole body, which then becomes contorted into strange attitudes. Most frequently the temporal or masseter muscles are affected in mild cases, or the whole head may exhibit a jerky side-to-side movement; in other cases, one leg may show a jumpy or extravagant action when the dog progresses, or regular spasmodic flexions when at rest. When the intensity of the spasms increases, they are noticed equally as well during sleep as when awake; in fact, they often become so violent and continuous as to prevent the animal from sleeping, and so exhaust the patient that death eventually ensues. In the human being, it is most common among children, and is closely associated with acute rheumatism; in many cases also, the heart shows signs of an endocarditis similar to that observed in acute rheumatism. The so-called chorea in adults is said to be habit-spasm only.

This affection, however, is not necessarily a fatal one, and may after a lengthy duration gradually diminish and finally pass away, though unfortunately it has a tendency to increase in severity and terminate in fatal convulsions. Dogs sometimes live for several years affected with chorea, which then may or may not disappear entirely; thus it is an imprudent and faint-hearted procedure to abandon all hope of recovery and condemn a dog to an untimely death, especially where the animal is of considerable value either in a sentimental or monetary sense.

A poor man might probably be well advised to follow such a course in view of the very problematical prospect of a complete return to usefulness, and the cost of administering treatment over a protracted period; also, in arriving at a.decision, the possibility of a paralysis 
supervening must not be lost sight of-the animal becoming a complete or partial cripple.

Gray, however, records a case in which a dog, having suffered some years from chorea, then became paralysed, but eventually absolute recovery resulted.

Chorea affecting any muscle or group of muscles may be succeeded by paralysis of those parts, extending ultimately, perhaps, to general paralysis, coma, and death. By judicious treatment, however, it is often possible to considerably lessen the spasmodic activity and its injurious effects by enhancing the general tone of the nervous system and building up a strength which might, even permanently, overcome the morbid condition.

It has been said that the name "chorea" as applied to this affection of dogs is a misnomer, as the movements (incessant snapping of jaws, twitching of eyelids, and jerking of the head from side to side) are not of the same order as those in chorea of children, but are indicative of cortical irritation. In true chorea of man there is found no pathological alteration of the brain; anæmia is present, and spasms are not confined to special muscular groups, but various muscular centres of the body are implicated.

Cause of Chorea.-Chorea in dogs, although it might possibly exist independently, is very constantly associated with distemper, and in fact is declared by some to be absolute proof of the present or previous existence of that disease. Nevertheless, it is seen also in myelitis of traumatic or other origin, and as a result of abscesses and tumours contiguous with the brain, etc. In distemper its most probable etiology is the action of bacterial toxins on the grey matter of the spinal cord, for the inferior horns have been observed by some to be thickened or hardened. Carougeau, Bohl, and Rexter noted an infiltration of leucocytes in the grey matter of the entire cord, especially in the inferior horns. 
Signs of a Previous Attack of Distemper.-It is certain that if a practitioner were asked to state definitely whether a dog brought to him suffering from chorea had comparatively recently been a victim of distemper he would, in the absence of any history, be sorely exercised to give an opinion. He would, however, be extremely suspicious that such was the case, and he might endeavour to confirm this view by searching for the few signs occasionally discoverable after an attack, such as deafness, defective sight (cataract, keratitis, corneal cicatrices, etc.),

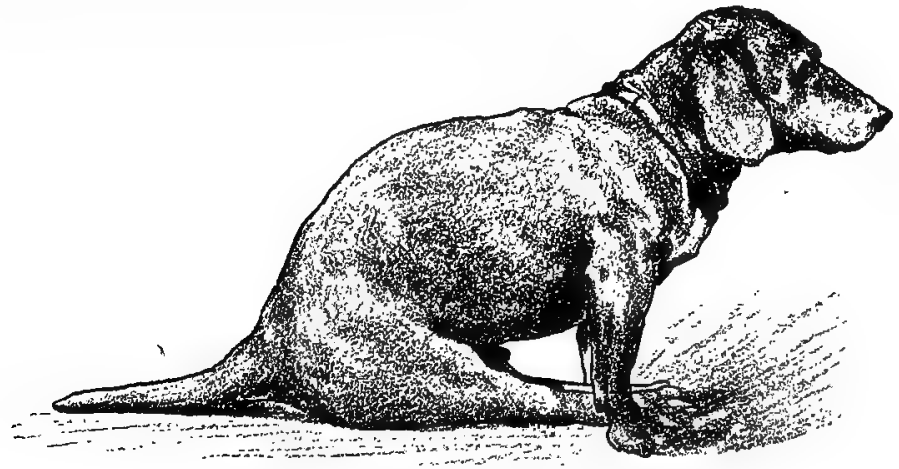

Fig. IO.-Paralysis of the CORD.

(Müller and Glass, " Diseases of the Dog.")

decay or erosions of dental enamel, and the black or dark brown pigmented areas which may be found on the abdomen, thighs, ear-flaps, or other parts, marking the spots where pustules had previously been, and assuming a size ranging from that of a pin's head to a pea.

The Paralyses-Paralysis.-Paralysis is a diametrically opposite condition to convulsions, and may be described as a loss of motion or sensation in a living part or member, as opposed to the diseased involuntary contractions of the same parts when affected with convulsions.

Paresis.-A distinction may be drawn between paralysis, or entire loss of movement, and paresis, or partial disability, as evidenced by lameness or weakness from 
debility. As regards the origin of complete paralysis, the various parts of the body are controlled by certain centres in the brain, and any disease or abnormal condition which becomes located in this cortico-muscular brain centre, and inflames or inhibits the power of these centres, must involve a paralysis of the muscles which they control.

These provocative lesions may owe their own origin to traumatism, inflammation, or the compression caused by inflammatory exudates, or actual degeneration of the

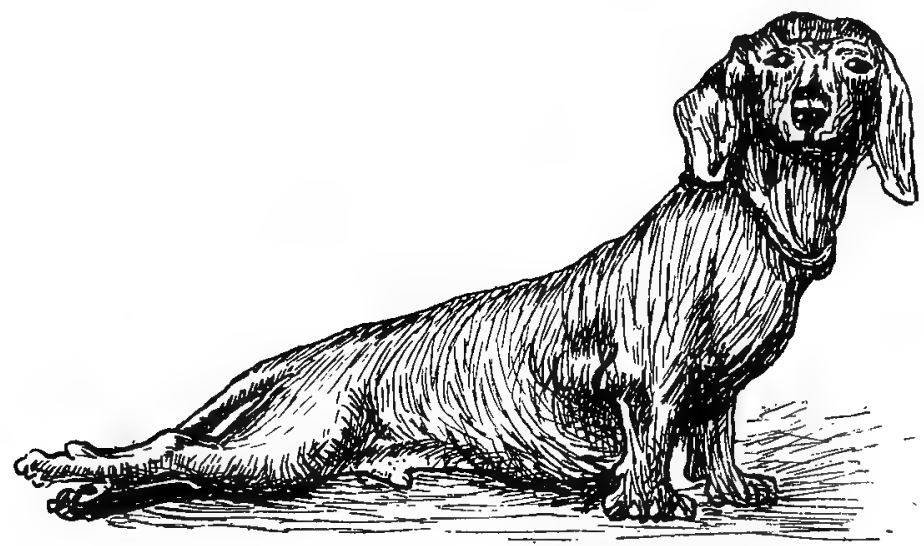

Fig, it.-Paralysis of Posterior Extremities. (After Müller and Glass.)

tissue itself. Pathological conditions of the brain coverings sometimes cause a paralysis of one part of the body, such as of the lower jaw, or one or other of the limbs, etc. In the former affection all power of opening or closing the jaw is lost, and mastication becomes therefore impossible; the tongue hangs out, and saliva dribbles from the mouth.

Paralysis is a frequent symptom of rabies, though somewhat rare in distemper. In paralysis of the limbs the affected leg or legs are never used, but are dragged along in a limp or flaccid state. 
Paraplegia.-Paraplegia is a paralysis of the hind-legs and hinder part of the body, affecting both motion and sensation, the condition being indicative of disease or injury of the spine. The dog remains strong and active in his fore parts, but owing to the debility or total disability of the hinder parts, he totters during progression, or may drag the inert remainder of his body after him. The paralytic affection may in other cases be more universal, and cause him to reel about as if intoxicated.

Hemiplegia.-In hemiplegia, paralysis is observed in the muscles of one side of the body only, as a result of disease or injury in the brain on the opposite side. None of these paralytic states need be regarded as necessarily fatal, and, indeed, they often prove to be only temporary conditions; but I hasten to disabuse the reader of any idea that they can be lightly regarded, since even where voluntary control of the muscles is regained there is always a possibility of permanent muscular atrophy limiting power and creating disfigurement.

Muscular Atrophy.-Where the condition is comparatively quickly amenable to treatment, little or no atrophy takes place, and the same may be said of paralyses arising from a cerebral source. Muscular atrophy, however, is a feature of spinal paralysis, and the degree of diminution will be an indication of the duration of the disease.

Sequelo of Paralysis.--Other phenomena occasionally noticed as sequelæ of paralysis are loss of sight, hearing, smell, and even voice, mental deficiency, and respiratory whistling.

Opisthotonos.-What has always proved a fatal symptom is the very distressing condition known as opisthotonos, in which the head is bent over the back by tetanic spasm. It is attended by periods of partial or 
complete unconsciousness, the animal being unable to feed, and the rigid contortion is maintained for hours and sometimes even days.

In my experience it has always terminated in deep coma and death, the patient's temperature being subnormal and the pulse hardly discernible.

Spinal Meningitis.-Arising in connection with toxic diseases such as distemper and rabies, one sometimes, though rarely, observes cases of spinal meningitis in which the pia mater is the membrane most affected, and paralysis is occasioned-chiefly of the posterior extremities, but sometimes also of the anterior. Consciousness is practically never lost, but hyperæsthesia may be marked, the animal constantly gnawing at certain parts of its body, and the penis in a constant state of erection; owing to contraction of the sphincters the evacuation of urine and fæces may be impeded, and the animal will howl or show other evidence of pain when touched, especially along the back.

At later or more serious stages the sphincters of the bladder and anus may also become paralysed, and the animal be subject to incontinence.

If and when the animal becomes moribund, the skin when gathered up in one's hand remains so for a few minutes, having lost all its power of contractility, and this is always to be regarded as a very ominous sign.

IV. Exanthematous Symptoms.-A great number of the earlier authors regarded the cutaneous eruption sometimes manifested in cases of distemper as a true variolous disease, or "dog pox." This was disproved by many experimenters from the fact that vaccination with cultures prepared from the pustules produced pustules and little else, and conferred no immunity; also from the fact that these cultures have practically always given pure growths of staphylococci. 
The exanthema of distemper cannot be regarded as having a separate entity, but as a secondary infection which may or may not be present. Two distinct opinions are current as to the diagnostic value and frequency of a skin eruption in this disease, each upheld by a formidable array of supporters: one school declaring that the eruption is of first-rate importance, Trasbot even going so far as to look upon it as the primary infection, all others being subsidiary; the other as stoutly maintaining that, if it appears at all, it is of little or no significance.

Gray, in his paper on "Canine Distemper" (Central Veterinary Society, 1905), gave it as his opinion that distemper was essentially an eruptive disease, and that broncho-pneumonia was entirely a secondary symptom. He quoted no less than about fifteen other observers who were agreed as to the eruption being present, and who pronounced it an "eruptive disease." Sewell, however (Veterinary Record, I905), says: "I would like to state that in my experience, now extending over thirty years, it is the exception and not the rule for a canine distemper patient to have an eruption on the skin. In cases where it does occur it is generally noticed in young puppies."

Mayall (Veterinary Record, I905) added: "In my experience, distemper as a primary skin eruption hardly has any existence." M'Gowan (Veterinary Journal, I9I2) remarked: "I have only seen the rash three times in $200 \mathrm{dogs}$, and it is not, in my mind, of the slightest importance in diagnosis, as it occurs so seldom, occurs late in the disease, and in a situation where irritation of fermenting urine and decubitus cannot be excluded as the most potent factors in its production." Ferry records only eight cases showing skin lesions out of sixty-eight animals suffering with distemper; and my own clinical observations (extending now over thirteen years and entailing the handling of probably some hundreds of distemper cases) are in entire agreement with the last- 
named gentlemen. A constant vigilance has been maintained for cutaneous no less than other lesions, but the results have been almost negative, since only about 8 per cent. of cases of distemper have been found accompanied by a rash.

Suggestions have been put forward that the eruption is frequently overlooked or not observed, in consequence of the practitioner not, as a rule, being called in until the disease is far advanced, by which time the eruption has generally vanished; but serious objections can be raised to that theory in that ( $I$ ) healing takes place with desquamation of the epidermis only after about seven to eight days from its onset, at the earliest, and even then leaves discernible traces behind; (2) many veterinary surgeons who possess boarding kennels or infirmaries in which dogs remain for long periods, have ample opportunities of watching cases of sickness from their very inception, apart from those which they visit at their owners' homes; and (3) in many instances the cutaneous symptoms are not manifested until at or near the termination of the primary malady.

I knew a case in which a Pekingese puppy was the subject of an extensive rash for nine weeks, and eventually died.

It is held by some authorities that this eruption is the very earliest symptom of distemper, the spots appearing and disappearing within forty-eight to sixty hours; thus if the animal were not under suspicion they might well be overlooked. I can remember no cases of this kind, but have found skin lesions appearing for the most part during convalescence or about the crisis. In a very small minority of cases they have occurred in the absence of any symptom other than dullness and malaise, leading one to doubt whether distemper was implicated or not.

Diagnostic Value.-It was stated earlier in this work that the eruption is characteristic of distemper, and if the 
two statements appear conflicting I would explain that in the vast majority of cases where a vesicular eruption appears, other typical symptoms of distemper can also be apprehended, which leave no doubt about the diagnosis. In other words, when this peculiar eruption is encountered it is practically always in association with distemper, and becomes in consequence characteristic of it. Nevertheless, it occurs both seldom and late, and cannot on that account be credited with any primary importance.

Predilections.-Very young puppies are the most susceptible, though middle-aged or old dogs are not entirely exempt. Where it is preceded by a slight rise of temperature, the latter usually drops to normal upon the advent of the eruption. The predilection seats are the thin-skinned hairless parts of the body, such as the abdomen, inside the arms and thighs, scrotum, inside of the ear-flaps, and rarely on the back or head.

Characters of the Lesion.-The lesion commences as a small circular red spot like a flea-bite, which later (say, one day) becomes elevated into a nodule surrounded by a red ring, and may, during the next three or four days, attain the circumference of a pea or even that of the head of a lady's hatpin. During this period the nodule has become vesicular, and contains a yellow serous fluid, which infrequently changes to a purulent character. About the fourth day the vesicle usually ruptures, forming a moist, sticky sore, which becomes scabbed over; or it may subside without breaking and wither up, the whole lesion now being surrounded by a circular red area with a brownishyellow crusty centre, which peels off in large scales.

When the vesicle becomes a pustule, a deeper layer of skin is involved, which inevitably leads to a more severe and visible disfigurement of the skin after resolution has taken place. Usually the lesions are few in number, but may vary from a solitary spot to multiple spots all over 
the body. They give rise to an unpleasant odour; but occasion no pruritus worth mentioning, and in that respect are easily distinguished from any condition set up by mange parasites. At first thought it might be considered difficult for a trained eye to confound mange with a distemper eruption, since the lesions of the latter are practically always discrete; but there are at times cases in which they become confluent, forming large scabbed

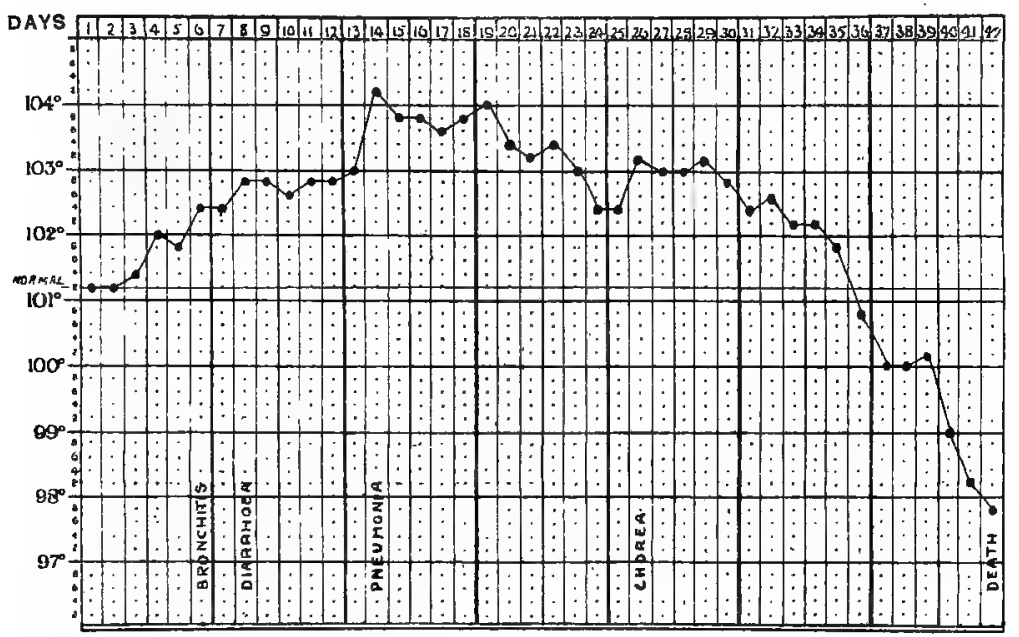

Fig. i2.-Temperature Chart of a Protracted Fatal Case, SHOWING INCIDENCE OF COMPLICATIONS.

or moist matted areas, and setting up more or less intense irritation. Such instances, however, are very rare.

The localities actually affected by the eruptions become permanently denuded of hair, and traces of the former may be found long afterwards in the shape of black or brown pigmented, shiny, crinkled areas, each marking the seat of a previous vesicle.

Febrile Symptoms. - If symptoms of uncomplicated distemper were the only ones present in a typical attack, there would probably be little or no observable tem- 
perature; nevertheless, as in most instances the true disease is complicated by more or less severe symptoms, pyrexia generally supervenes. In some cases the temperature is fairly steady, whilst in others it is irregular, rising or dropping without apparent reason. If bronchitis becomes severe, or if enteritis appears, the temperature will rise in proportion to their intensity, and still further rises when pneumonia develops, reaching, perhaps, as high as $106^{\circ} \mathrm{F}$. There have been even fatal cases in which no pyrexia has been observed from beginning to end, though these instances are undoubtedly

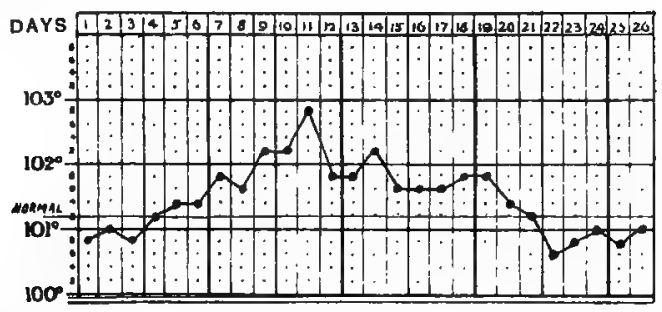

Fig. I2a.-Temperature Chart of a Benign Case.

rare; for the abnormal temperature may have existed so early in the attack, and unaccompanied by other symptoms, that nothing amiss was suspected, and therefore no record was taken.

At the onset of a secondary infection of some important organ, a rise of temperature is usually noted, and where the temperature throughout has been only slightly in excess of normal, such an elevation occurring suddenly would be a grave sign.

The temperature in most cases of distemper is not a high one, though should the malady be accompanied in the early stages by a steadily maintained high temperature this is not necessarily an indication of grave consequences, but, on the contrary, foretells, as a rule, an eventual recovery. Thus it is better to encounter a bigh 
temperature in the early stages than that it should appear late in the disease.

Subnormal Temperatures.-It is not uncommon to find the temperature has become subnormal after having reached its highest limit, a phenomenon which is accompanied by a reduction in the number of heart-beats and respirations, restoration of appetite and vivacity. On the other hand, should the subnormal registration supervene upon an attack which has throughout been characterised by some severe protracted symptoms, high temperature, emaciation, great exhaustion, and semicoma, it is an infallible sign of impending death. The pulse becomes then almost imperceptible, respiration rapid and shallow, and the temperature may only be $95^{\circ}$ or $96^{\circ} \mathrm{F}$. The animal is weak, anæmic, perhaps even paralysed and collapsed.

Miscellaneous Symptoms-Emaciation.-Emaciation, in greater or less degree, is practically inseparable from distemper, and where it gains a firm hold it bodes ill for the patient's prospects of recovery. There is always a certain amount of wasting, even in those cases which are of the benign and transitory type, and it is sometimes difficult to explain why there should be. I have known it to become very marked in dogs which have been most liberally fed on the best of nourishing foods, and which have never had a temperature higher than $102^{\circ} \mathrm{F}$. during the two or three weeks' course of their attack. It is to be feared where its development is rapid, and the accompanying diarrhœa of a very stubborn exhausting nature, and there is a perceptible increase of weakness, together with the peculiar obnoxious and characteristic body odour. These signs are prognostic of a fatal termination.

Muscular Symptoms.-Rarely, symptoms pertaining to parenchymatous degeneration of the cardiac muscle 
appear in distemper, which, through the resultant weakness of the heart, generally sets up œdema of the lungs and death.

Symptoms simulating rheumatism have been observed by some authors several days prior to the manifestation of typical diagnostic symptoms, whilst muscular weakness frequently impóses itself some time after their advent. This weakness is common to most, if not all, febrile conditions, and as the dog realises its inability to stand or walk without staggering, it remains preferably recumbent, thereby probably increasing rather than diminishing its intensity.

Peritonitis.-Peritonitis, as its name implies, is an inflammation of the peritoneum, which may be localised in the beginning, or later become diffuse. It may arise from ulceration of the bowel, from extension of the inflammation of the mucous to the serous coat of the intestine, from pyæmia, or occur from a general inflammation of all the serous membranes of the body. It is marked by the secretion of an exudate, which may be serous, fibrinous, purulent, or hæmorrhagic, and the peritonitis would be named according to the character of the exudate.

In the first stage the peritoneum becomes congested and reddened, later exuding a serous fluid, the fibrinous components of which form a layer on the peritoneum, which has the effect of causing adhesions of bowel to abdominal wall, or of bowel to bowel. One may forecast the age of the lesion by the degree of tenacity with which the various organs or parts adhere to one another-i.e., if the condition is recent, the adhesions easily break down; old ones are difficult of separation.

Varying amounts of liquid collect in the abdominal cavity, and the serous walls will be found œidematous and friable. The abdomen is tense and firm, and the dog resents its manipulation, crying out with pain if 
touched. The back is arched, the animal dull and stiff, vomiting of a greenish-yellow mucus frequent, and the appetite completely in abeyance. The temperature, if previously fairly low, may rise to $104^{\circ}$ or $105^{\circ} \mathrm{F}$., but usually stops at the former figure.

Later, the abdomen may become distended from the presence of accumulated fluid, and this helps materially in the diagnosis of the condition; but when the forma-

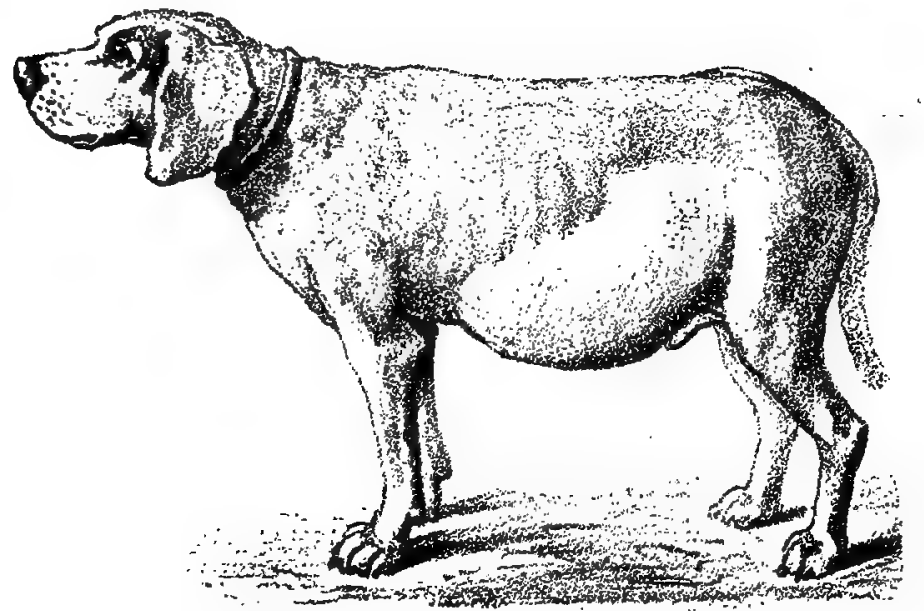

Fig. I3.-Dog virh Ascites, ARIsing sometimes from Peritoxitis. (Müller and Glass, "Diseases of the Dog.")

tion of this serosity maintains a gradual increase, a pressure is exerted upon the diaphragm which seriously impedes the lungs, and produces sometimes acute dyspnoa and frequently even death from either suffocation, heart failure, or collapse. The pulse has then become thin and rapid or practically imperceptible.

Peritonitis is always very fatal, but more rapidly so where it is due to perforation of the bowel wall, followed by septic infection.

Suppurations.-Some authors have described pyæmic lesions in connection with distemper, but personally I 
cannot recollect having seen a single case of this kind. Abscesses have been observed in various parts of the body, particularly in the parotid or submaxillary regions, about the eyes, lips, forehead, jaw, shoulder, or inguinal and umbilical regions. The swellings are said to have attained the size of an orange or cocoanut, their contents being of a thick yellow or cream-coloured purulent or hæmorrhagic character. The condition is sometimes associated with a suppurative process of the laryngeal or pharyngeal regions, or even of the internal organs. "Healing is difficult, and frequently a fistula remains in communication with an extensive abscess cavity; especially in the region of the throat" (Gray).

Suppurations of Anal Glands.-Occasionally a dog may become the subject of a suppuration in the anal glands; or their ducts may become occluded, thereby producing swelling and irritation of the anus, resulting in obstructive constipation. The animal evinces its discomfort by chasing or biting its tail, or sliding its anus along the ground in its attempts to relieve the irritation. Upon pressing the swollen glands a thick purulent material exudes, the evacuation of which affords the dog great relief.

Otitis.-Otitis, or inflammation of the ear, occasionally accompanies or follows distemper, and in such cases arises probably as an extension of infection from the pharynx via the Eustachian tube, an otitis media being produced. The symptoms exhibited are whining and restlessness with a frequent scratching at the ear and desire to rub it against any fixed object: Attempts by the surgeon to manipulate it, however, are rigorously opposed, unless the inflammation is early and of mild character, when the dog will usually welcome the rubbing or handling of the affected ear. Sometimes the inflammation becomes acute, and a copious purulent discharge 
(otorrhœa) is in evidence. There is great pain and some amount of deafness; though, unless both ears are affected, it is often difficult to decide to what extent hearing has been impeded. It is only in very rare instances that these cases will not yield to adequate treatment, although in the severer forms permanent deafness may result.

Balanitis.-Inflammation of the glans penis is rarely seen in distemper, but when present might be mistaken for preputial catarrh, which is rather more common.

In these conditions there is swelling of the glans or prepuce, whichever may be the seat of affection, and a yellow purulent discharge drips from the end of the prepuce. The dog calls attention to it by constantly licking the part, or wet stains may be left wherever the animal has rested for any time. . It is not of serious consequence, and although frequently attributed to a specific origin, the dog does not 'suffer from specific gonorrhœa, and the urethra is not implicated. A similar condition may rarely be observed affecting the vulva of the bitch.

Nephritis.-Probably affections of the kidney are more constant in distemper than is generally realised, owing to the fact that in slight inflammations little or no disturbance of health in the dog is apparent.

Diagnosis would be rendered more certain by microscopic examination and testing of the urine voided, and to distinguish between the various types of nephritis one must place most reliance in the post-mortem examination of the kidneys.

This complication arises from the absorption of the toxins of the primary specific disease, and is generally of a chronic catarrhal character, in which the pyramids are of a reddish hue from the plugging of the tubular canals with cells, and the cortical portion yellow or yellowishwhite. It may arise subsequent to recovery from dis- 
temper on account of the irritation set up by the injudicious administration of phosphorus or arsenic used in the treatment of chorea, etc., or from absorption of turpentine or cantharides used as counter-irritants in various conditions, in which cases the resulting nephritis would be of the acute type.

Symptoms will be evident in cases where the condition is severe, and as a first indication one may notice a rather more frequent micturition with the sometimes painful passage of only a small quantity of dark-coloured urine each time. It may even be blood-stained or contain clots of blood (hæmaturia).

Miller, in his "Practice of Surgery," says: "The renal source of the hæmorrhage is known by the blood being diffused equally through the urine; by the expelled fluid containing cylindrical portions of fibrine, like small worms-the result of coagula in the ureter-sometimes colourless and sometimes pale-pink; by the appearance of blood, being preceded and accompanied by pain and heat in the loins, and other renal symptoms."

Owing to the diminution in the amount of urine passed, dropsical conditions of other parts ensue. Constipation is present. Dogs walk with an unusual stiffness of the hind-legs, whilst bitches assume a crouching attitude as though about to urinate. Pain is caused by pressure over the loins or by palpation in the region of the kidneys, and the latter may be found to be enlarged. Vomiting is often observed at the outset, and the dog loses all desire for food, if such has not already been lost.

Examination of Urine.-The urine, if examined, will be found turbid, of increased specific gravity, and çontaining an excess of albumin, some red and white blood corpuscles, or white alone, and tube-casts, the discovery of which should assure the investigator that some kidney disease exists. 
Symptoms in the later stages would be those of subnormal temperature, weak thin pulse, probably diarrhœa, and in fatal cases suppression of urine, convulsions, coma, and death. If resolution is taking place, the amount of urine passed will gradually increase to normal, and it will be clearer and contain fewer foreign bodies.

If the nephritis is of a diffuse chronic type, the kidney becomes small, cystic, nodulated, and adherent to its capsule. The interstitial tissue is increased, and vessel walls and Malpighian corpuscles thickened. The amount of urine voided will be increased; it will be pale in colour, and will contain albumin and casts, though a smaller amount of the former than in acute nephritis. The pulse will be hard and full at first, perhaps becoming weak and accelerated as the disease progresses, and when the latter occurs the urine passed will be again diminished and darker in colour, and death may eventually result from uræmia, or the pressure on vital organs produced by generalised dropsy (anasarca). Nephritis, although grave, is not necessarily fatal in dogs, though its early diagnosis is essential in order that certain medicinal agents and foods which would prove harmful may be avoided.

It is common enough, in post-mortems made upon aged dogs which have died from natural causes, to find extensive kidney disease which during life was never suspected, and which never appeared to have disturbed the dog's health in any way.

Cystitis.-Distension and inflammation of the bladder are quite common in distemper, especially when nervous complications are also present. Retention of urine is, in such cases, probably occasioned by weakness of the muscular coats of the bladder due to chronic catarrh or paralysis. Cystitis may be caused by extension of inflammatory processes from the kidneys or ureters, or 
by infection with various micro-organisms, particularly the pyogenic cocci, and the $B$. coli communis. Animals which have become greatly debilitated, or which are in the last stages of a fatal attack of distemper, very frequently exhibit cystitis upon post-mortem examination. The condition is often unnoticed in life; indeed, it is fairly effectually cloaked by the symptoms arising

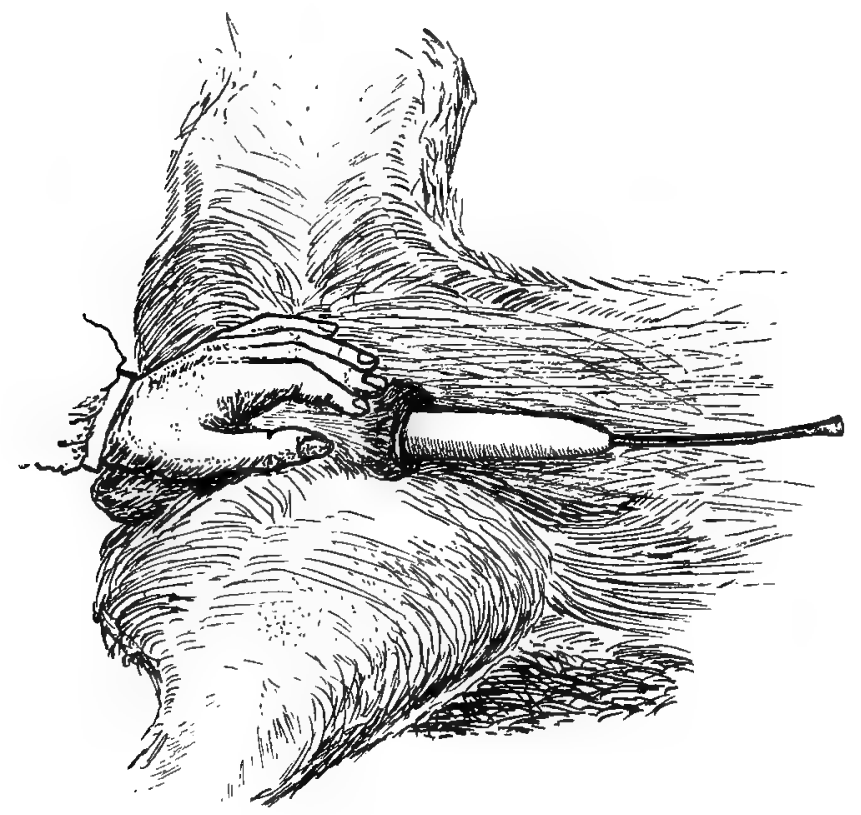

Fig. I4.-Passing the Catheter.

The prepuce must first be drawn back.

from the other numerous complications of the attack, unless severe in character.

A dog may appear to void urine in an apparently normal manner throughout, never leading the attendant to suspect suppression or the necessity for passing a catheter; but upon autopsy great vesicular distension is not infrequently discovered. The urine passed is merely an overflow, the bladder being no longer able 
to accommodate any increase, and sometimes rupture of the organ occurs with rapidly fatal results. The symptoms produced in cystitis are similar to those observed in nephritis, the animal making frequent

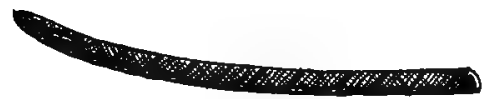

Fig. I5.-Male Vesical Catheter.

attempts at micturition, though passing, perhaps, only small quantities or even none at all.

A moderate intermittent temperature may be recorded, and pain evinced on pressure of the flanks. Micturition

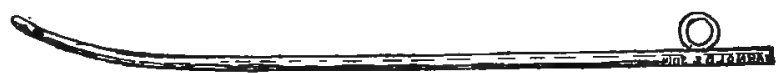

Fig. i6.-Female Vesical Catheter.

is always a painful process, and the animal sometimes cries out during its performance. A differential diagnosis may be arrived at by testing the urine and noting the absence of tube casts and albumin; by observing also

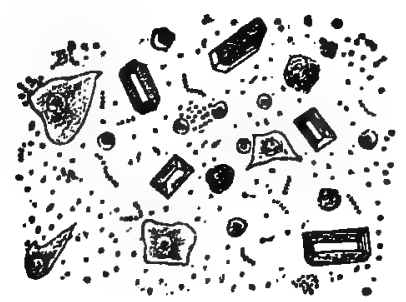

Fig. 17.-Urine of a Dog with Cystitis, showing Cocci, Crystals, and Epithelial Cells.

(Müller and Glass, " Diseases of the Dog.")

that the urine rapidly ferments and develops an ammoniacal odour. The microscope will also reveal the presence of crystals of phosphates, numerous bacteria, epithelial cells, and, in rare cases, uric acid crystals.

The malady is usually a curable one, though recur- 
rences are not infrequent under conditions which favour them. Failure to detect and treat distension of the bladder will lead to a loss of its contractile power, and symptoms of incontinence may then be manifested, which for this and other reasons may endure for months, and, in the case of an old dog, perhaps for the remainder of its lifetime.

Hydrocephalus. - This sequel is rarely seen, though it is known to have followed distemper. Frohner reported having diagnosed it in only twenty-nine cases out of seventy thousand dogs which passed through his hands in Berlin. The dog becomes a complete idiot, progressing with a staggering gait, holding the head to one side, staring stupidly at objects it encounters, hiding in dark corners, listless and defective in sight and scent, and presenting a pathetic picture of its former self. Sometimes large amounts of fluid are found in the lateral ventricles of the brain on post-mortem examination.

It will be realised from a consideration of these manifold symptoms how completely the whole body may sometimes be implicated in this dread disease, and yet at others how extraordinarily benign the attack may be. 


\section{CHAPTER IX \\ COURSE, PROGNOSIS AND MORTALITY}

COURSE.

To attempt to define, with any degree of precision, the course which distemper will run, would only mislead the reader and misstate the actual facts; but in the great majority of those cases which recover, the disease will run its course in three to four weeks from the onset of symptoms. There are, however, so many factors which influence both the prolongation and termination of the malady that variations from the rule are frequent, and one can never be sure at the beginning what time must elapse before the end.

Some cases seem so mild as almost to escape attention. Thus, beyond a slight indisposition with an occasional cough, nothing more is observed, and the animal has regained its normal health inside of seven days. At other times a dog appears to contract a virulent attack, to which he may succumb in three days or even less. The vitality of the animal at the time of infection plays an important part, for where his natural defensive powers are good he will probably only take the disease in a mild degree, and recovery may be complete within about a fortnight. If, on the other hand, he is debilitated from any cause, his body will be unable to withstand the invasion of pathogenic organisms, which, meeting with little or no resistance, are enabled to multiply at an alarming rate.

Secondary complications supervene rapidly one on the other, and the dog is soon in the throes of a sickness 
which may drag on for several weeks or even two or three months. It is regarded, I think pretty generally, that not only a lengthy course but also a fatal termination may be ascribed entirely to the secondary infections. Thus, the nature of these infections and the virulence and dose of their causal organisms will materially affect the length of time during which an animal may remain ill.

Where nervous symptoms are evinced, these may remain long after all other traces of distemper have entirely disappeared; in fact, paralysis and chorea sometimes persist during the remainder of the dog's lifetime.

In cases which are complicated with a severe attack of pneumonia, one would not expect to find complete resolution in less than five or six weeks, which period may frequently be extended into three months.

\section{PROGNOSIS.}

One can never be too guarded in forecasting the probable termination of an attack of such a treacherous disease as distemper, for however benign the attack may appear to be there is always a possibility of the sudden appearance of very severe complications, which may carry off the patient at short notice.

Generally the prognosis is unfavourable, owing to the high mortality, and optimism as to the result should never be indulged in. Cases complicated with catarrhal pneumonia or nervous symptoms must always be regarded very seriously, and the dangers are increased as the intensity of these symptoms is accentuated.

Prognosis is usually more hopeful when the animal attacked is fairly mature-say six to twelve months of age-a mongrel, or one of the hardier breeds used to taking plenty of exercise, such as the Irish or fox-terrier, Sealyham, Airedale, etc. Delicate breeds and very young 
pups are far less able to withstand the infection, and they succumb in greater proportion.

Dogs habitually fed on an artificial diet, which includes very little or no meat, may also be expected to suffer in greater degree than those living on flesh. Pneumonic lesions are undoubtedly present in the majority of cases, and on account of the liability to extensive pulmonary solidification or œdema, must be considered as very grave.

A sudden and marked drop in temperature, unaccompanied by any diminution of the distressing symptoms, is to be looked upon as an exceedingly sinister phenomenon ; and conversely, where a sustained high temperature supersedes upon what in the beginning was a normal or nearly normal temperature, it is a serious indication of the development or incidence of a grave complication connected perhaps with the lungs, nervous system, or alimentary tract.

Whilst the nervous manifestations usually arise late in the disease, convulsions are nevertheless sometimes seen in the early stages, when they are not regarded quite so seriously, especially if the fits are few in number. When, " however, they appear towards the end of the malady, prognosis becomes more than ever doubtful, death being a too frequent termination.

Chorea is not necessarily lethal, but it often renders an animal so useless and miserable that an owner prefers to have the dog destroyed rather than witness its evident distress. Youatt remarks: "One fit is a serious thing; if it is followed by a second within a day or two the chances of cure are diminished, and if they rapidly succeed each other, the dog is almost always lost."

In some cases the nervous symptoms are succeeded by general paralysis and death in a state of coma; death is then probably produced by paralysis of the brain. 
It is a much more favourable sign to see resolution effected gradually rather than suddenly, for frequently when a spontaneous cessation of pulmonary, intestinal, or other symptoms is observed, such apparent rapid improvement may unhappily be followed by the onset of the graver nervous phenomena, indicating a transference of the disease by metastasis to the brain.

Change in the weather exerts an undoubted influence on animals affected with distemper, for not infrequently what had promised to be a favourable case will become an acute one at the onset of adverse climatic conditions; therefore patients must as far as possible be kept in an equable temperature, and protected against all such atmospheric changes.

The presence or absence of marked emaciation is a very important factor in connection with the prognosis, for where an animal is greatly emaciated, little or no hope can be held out of its recovery. Profuse and persistent purgation will rapidly produce such a condition, and for this reason must be regarded with great apprehension. On the other hand, let the diarrhœa be ever so violent, if the animal's bodily condition can be sustained, one may feel tolerably confident of the possibility of recovery. Other severe symptoms may even abate, yet if the loss of flesh be unremitting, the termination will be invariably fatal.

The onset of jaundice portends great evil, and diminishes the prospects of recovery to practically nil. The dog falls away more in twenty-four hours than would have been thought possible; his bowels are obstinately constipated; he will neither eat nor move, and in two or three days he is dead.

Caries of the ethmoid or turbinate bones, as evidenced by a foul-smelling sanious and darkened nasal secretion; a singular fœtid odour emanating from the dog; the fæcal 
matter consisting solely of blood and mucus with an unbearably evil smell; sunken eyes, and the vomition of a putrid brown-coloured fluid, are all conditions which lead one to predict with fair certainty the hopelessness of the case.

Some writers consider the appearance of pustular lesions occurring late in the disease as a grave sign, whilst others regard it as particularly favourable, Youatt giving as his reason that "the disease is leaving the vital parts and expending its last energy on the integument." Personally I attach no significance whatever to the cutaneous symptoms, these being usually absent, or, when present, seeming to affect the course of the malady very little either one way or the other.

Perhaps, on the whole, I can remember more cases which have recovered than which have not, among those dogs whose skins were implicated, though what influence the exanthema exerted, or could exert, I can'not say.

In distemper, as in every other disease, relapses are to be feared, for whilst a patient may emerge safely from the initial attack, death is very often the sequel to a second infection. It must not be forgotten that although an animal may reach the stage of resolution, it is nevertheless still liable to become a subject of one or other of the secondary complications-or rather, sequelæ-such as chorea, paralysis, epilepsy, etc., even as late as several months afterwards; so it becomes apparent that the versatility of this treacherous disease must baffle, from beginning to end, all attempts at prognostication, leaving us as dubious at last as we were at first. 


\section{MORTALITY.}

True to the characteristic variability which marks this disease from every aspect, the death-rate fluctuates, being greater in some seasons or in some outbreaks than in others, and less in country districts than in large cities. The average mortality may be assessed at 25 per cent. Müller and Glass estimate it as between 60 and 70 per cent. in big cities, and at 20 to 30 per cent. elsewhere ; Youatt computes it at 33 per cent.; Millais, 6o to 90 per cent., and of the highly-bred, 90 per cent.; Gray, 25 per cent. ; Ferry, 90 per cent. among pure-bred dogs in New York, and 60 to 80 per cent. among ordinary street mongrels; Friedberger and Frohner, 50 to 60 per cent.

These discrepancies arise doubtless from the fact that some of these authorities have based their figures upon the results of certain epizoötic outbreaks of a virulent type, whilst others have probably taken an average spread over many years of practice, thus embracing sporadic cases in addition to those of epizoötic origin. I do not think any general practitioner to-day will estimate the death-rate higher than approximately 25 per cent.

The age and breed of animals affected exert a strong influence on mortality, very young puppies or aged adults being far more apt to die than animals between the ages of four and twelve months and up to five or six years. Similarly, highly-bred animals, and those exotic to a country, will perish in much greater proportion than the common mongrel or indigenous breeds. Among the latter again we find some breeds are more highly resistant than others, such as fox-terriers, bull-terriers, Irish terriers, Airedales, spaniels, setters, etc., in which probably only about i 7 per cent. of cases prove fatal. Collie dogs, however, appear to be peculiarly susceptible to 
lethal attacks, as also do Alsatian wolfhounds, borzois, greyhounds, and Japanese spaniels.

Other influences adversely affecting mortality are changeable weather, the frequently virulent type of infection prevailing, and habitual improper feedingsuch as a diet of bread, vegetable, and biscuit.

Blaine correctly observed: "Wherever man has interfered in forcing an artificial breed, and in maintaining and perfecting a degree of forced excellence, there the disease is almost always severe.... Some breeds possess an hereditary tendency to have it worse than others of the same kind ; litter after litter of some sporting strains will hardly yield more than one or two survivors. In such a case I would advise the breeder to cross the race, or to altogether try a new one."

Where death is the termination to an attack, the direct cause may be attributed to cerebral paralysis, œdema of the lungs, pyæmia, septicæmia, or exhaustion. 


\section{CHAPTER $\mathrm{X}$ \\ MORBID ANATOMY}

THE lesions which may be encountered upon post-mortem éxamination are very numerous and varied, and whilst in a few isolated cases little or nothing will be found commensurate with the severe conditions established, yet in many others such a combination of lesions will be discovered as to force one, perhaps, to wonder how the dog could have survived so long. Pathological changes are mostly confined to the respiratory and alimentary tracts, although other organs are by no means infrequently implicated in addition.

Respiratory Lesions-Lungs. - In nearly all cases, the lungs are affected to greater or less degree, and the organs found to be darker in colour, consolidated in their lower third, two-thirds, or even in toto, and exerting a resistance to the knife when cut into. One or both lungs may be implicated, and areas of congestion may be noticeable on their surface. Sometimes hæmorrhagic spots are visible all over the organs; in other cases these are absent.

Lobular pneumonia is the type usually occurring in cases of distemper, and the whole lung is rarely affected, the inflammatory foci being localised in small areas, which later may coalesce and extend over the inferior third or half of the lung. The diseased part is hard and firm to the touch, airless, dull red in colour, and rather more prominent than the adjacent sound tissue. Section of the part produces a frothy, sanious, or brownish-coloured thick exudate, especially if pressure is exerted. 
The inflamed areas occasionally contain centres of suppuration, from which copious pus will exude upon incision. The small bronchi and bronchioles contain a frothy greyish or even blood-stained pus which mechanically impedes the entrance and egress of inspired air, and assists in the production of dyspnca.

In some cases, particularly in puppies, the lungs become swollen or generally enlarged, darker, very moist and prominent, and a serous exudate flows from the cut surface in great quantity. This is the condition of oedema, which consists in extravasation of serum from the blood-vessels into the alveoli, bronchioli, and bronchi, which it almost completely fills, thus excluding large portions of the lung from free circulation of blood and air, and producing in fatal cases cyanosis and death from asphyxia in from twelve to twenty-four hours.

As death approaches, profuse discharges of this liquid (rendered opaque by admixture with pus, and pink or red from the presence of erythrocytes) escape from the nostrils, an abundance of it also being found whipped to foam in the trachea.

Pleura.-More or less serous exudate may be present in the thoracic cavity, particularly where pleurisy has been a complication; and those portions of the pleura which cover the affected lung often become the seat of pathological change. The membrane may only show a roughening on its surface, or it may be covered with fibrinous lymph, which may cause the visceral pleura to adhere to the parietal, though this is rare. Congestion of the parietal pleura is sometimes observed, and it may even show a lymph deposit.

The nature and colour of the exudate found in the pleural cavity will depend upon the acuteness and type of the pulmonary disease; thus it may be hæmorrhagic, purulent, putrid and greenish, or serous. 
Bronchial Lymphatic Glands.-The bronchial lymphatic glands are not much increased in size, if at all, but frequently the cut surface is black or dark red; sometimes they contain pus and may be enlarged.

Trachea and Bronchi.-The trachea and bronchi do not commonly show any striking lesions, except, in a few instances, petechiæ; they do, however, frequently contain a frothy mucus, or a muco-purulent or bloodstained material, or both. The same may be said of the small bronchi, which, in addition, show a large amount of swelling and congestion of the mucous membrane. Ecchymoses or hæmorrhagic ulcers may also be present, and in the acutest cases all these conditions may be observed throughout the respiratory tract.

Larynx.-The larynx is usually found to be slightly inflamed, and may even be suppurating.

Nasal Mucous Membrane.-Petechial spots or ulcers are seen, in a minority of cases, on the nasal mucous membrane; at other times it is merely injected and purple in colour, or it may be pale and anæmic in appearance. Usually it is coated with an exudate of varying consistence; and where hæmorrhages or ulcers are present it will be sanious. This exudate is commonly yellow, and has a peculiar sticky property, which causes it to glue up the nostrils and cake around them in dry, hard crusts, which in turn frequently set up irritation or ulceration and impede respiration. Inflammation is very prone to attack the membranous lining of the nasal bones, and particularly the nasal portion of the ethmoid bone.

Alimentary Lesions-Ileum and Rectum.-In the digestive system we find the most common seats of pathological change to be the ileum and rectum, the cæcum and remainder of the bowel sometimes being also 
involved.' The mucous membrane of the two portions of intestine first mentioned may be found in a dry, corrugated condition, the ridges being highly inflamed and infiltrated with hæmorrhages, very similar to the condition seen in swine fever and rinderpest.

Intestines Generally.-Streaks of intense congestion may also be observed in other parts of the small or large intestine, but in most cases to a lesser extent. The entire intestinal mucous membrane is very swollen, tender, and pale, and the bowel lumen may be full of a thick, creamy mucus. If there are scattered hæmorrhages or ulcers, this material will be tinted a rosy pink, and, rarely, may be of a blood-red colour or contain pure blood. Fæces are usually absent. No change is noticed as a rule in the Peyer's patches, though they may be œdematous.

Anus.-I have frequently known the rectum and anus to be intensely inflamed, in which case attention is always attracted to the condition in life, when attempting to pass the thermometer.

Surgical Conditions.-Surgical conditions such as intussusception and prolapse of the rectum are infrequent, but when they occur they are often a prelude to a fatal termination.

Stomach.-The stomach, although irritable during the animal's life, is found on post-mortem to be very little changed. Sometimes, however, the gastric mucosa is slightly injected and swollen, especially towards the pyloric extremity. The corrugation or contraction, occasionally noticed, of the mucous membrane of the stomach and intestines is a condition consequent upon generalised emaciation brought about by sustained and profuse purgation.

Abdominal Lymphatic Glands.-Occasionally, though rarely, suppuration may be observed in one or other of 
the abdominal lymphatic glands. The latter may be cedematous or hæmorrhagic, but on the whole seem to be very little altered.

Peritoneum.-I have so far encountered no case in which peritonitis has played any part, and although it has been-known, it is rare. Nevertheless, a serous straw-coloured exudate is found not infrequently in small quantity in the peritoneal cavity, and this may on occasion assume the colour of bitter ale.

Liver. - The liver is generally found to be normal, but where diseased conditions have arisen, they are of the nature of hyperæmia (congestion) or parenchymatous hepatitis. In the first case a stagnating hyperæmia may be produced in consequence of the weakening of the heart's action, or of extensive pulmonary disease. The liver is then greatly enlarged, very hard, and overfull of darkened blood, the cut surface sometimes presenting a mottled appearance (nutmeg liver). In hepatitis we find the organ large, soft, and friable, no difficulty being encountered in pushing one's finger through its substance. Its colour is dark red in the early stages, becoming later of a clay tint, and the organ possibly smaller in volume. It then looks like liver which has been boiled or macerated.

Bile Ducts. - Where jaundice has been a complication, the mouth of the bile duct is frequently found closed, in consequence either of catarrh of its mucous membrane, or of swelling of the intestines in its immediate vicinity. In this contingency the gall-bladder is tensely full and its contents thicker than normal, or even semi-solid. Very little swelling is required to obstruct the bile ducts, those in the dog being so very minute, and when bile is unable to flow into the intestines it eventually enters the lymphatic system of the liver and gains the blood stream via the thoracic duct. 
This stagnation of bile no doubt accounts for the yellow staining of tissues adjacent to the gall-bladder, the biliary fluid having transuded through the coats of the latter under pressure. This localised staining is repeatedly seen on post-mortem examination of a distempered carcase, even in the absence of generalised icteric staining, but it is quite conceivable that sufficient obstruction could have taken place to force the bile to deviate locally from its normal path without setting up a well-established jaundice marked by a general discoloration of the body tissues.

Some authors have considered it a post-mortem change, a view which seems to be negatived by the fact that the condition can sometimes be observed upon opening the carcase very soon after death. All tissues of the body except the nerves, brain substance, and spinal cord, then become stained with the characteristic yellow colour of icterus. The liver itself may be any shade between yellow and dark brown, and, owing to irregular staining, may possess a mottled appearance.

Buccal Lesions.-In true distemper it is rare to find ulcerative lesions on the buccal mucous membranes; these, however, appear constantly in the allied complaint known as Stuttgart disease, in which, also, a necrosis and gangrene of the tongue are observed.

Gums.-The gums at their junction with the teeth are often injected and may be made to bleed quite easily. The mouth is of a purple colour, at one time dry and at another covered with a slimy, foul, stringy or hæmorrhagic saliva.

Tonsils.-Swollen, red, or purple-coloured tonsils are seen in a few instances, and in malignant distemper they may be coated with a diphtheritic deposit which also involves the larynx, pharynx, fauces, and soft palate. All 
these several organs may be œdematous, ulcerated, or ecchymosed.

Salivary Glands, etc.-The salivary and lymphatic glands of this region are not usually implicated, though swellings and suppurations have in rare cases been observed.

Heart.-The heart is not often the seat of pathological change, and the most one usually observes is injection of the vessels of the epicardium and pericardium, with moderate quantities of serous fluid in the pericardial sac. The auricles and ventricles, however, are frequently filled with black, tarry, blood clots, especially in the acute septicæmic types of distemper.

Kidneys.-The kidneys seldom become diseased to any great extent in consequence of distemper, and it is only occasionally that one discovers at autopsy an enlargement or shrinkage in size, softness or hardness, intense red or pale colour, the presence or absence of hæmorrhagic spots on its surface or cortex, and an easily detachable capsule or one tenaciously attached. More frequently one finds a moderate hyperæmia or congestion, which may in some cases have developed into nephritis. The type of nephritis most commonly associated with distemper is a chronic catarrhal condition in which the kidney is reduced somewhat in size, its capsule is more firmly adherent, and its cut surface shows a pale cortex in sharp contrast with a reddened medulla.

Muscles.-The muscles are usually wasted, and the fat seems to set imperfectly and remain soft and wet.

Central Nervous System.-Much attention has been bestowed upon the nervous system, microscopical and histological investigations having revealed various conditions which may be summarily described as follows :

Kolesnikoff discovered an infiltration by leucocytes of the brain substance and vessel walls, and a distension of 
arterioles and capillaries with red and white blood cells. Krajewski confirmed these observations and noticed also that the spaces adjacent to the blood-vessels were filled with lymphoid cells, even in animals showing no nervous symptoms prior to death. In acute nervous distemper Dexter and Mazulewitsch found changes in the vessel walls, the latter being surrounded by an albuminous exudate, which also invaded the interstitial tissue of the grey matter of the spinal cord; in chronic distemper they observed a localised interstitial myelitis, with partial atrophy of the spinal cord. Hadden found accumulations of blood corpuscles in it, and Trasbôt recorded that acute cases were accompanied by a considerable injection of the cord and its membranes with a sero-fibrinous exudate in and beneath the arachnoid, and even in the cord substance.

In a dog affected with chorea as a sequel to distemper, Carougeau noted an infiltration of leucocytes in the grey matter of the entire cord, especially in the anterior horn, which observations were confirmed by Bohl and Rexter. Little or no deviation from normal is often the macroscopic observation at autopsy, but many cases arise in which the membranes of the brain are found highly charged with dark-coloured blood, especially when epileptic fits have accompanied the disease.

There may even be a softening of the brain substance, with flattening of the convolutions, and a considerable effusion of serum into the ventricles. Blaine was of opinion that universal paralysis owed its origin to a morbid increase of the fluid in the ventricles of the brain.

The spinal cord as a rule appears normal, but in rare cases its sheath will be unusually vascular, softer in consistence, and suffused with more or less serous fluid.

Blood.-The blood may be deficient in quantity or quality, or even hydræmic, but in cases associated with 
some amount of septicæmia - and these are quite frequent-the blood takes on a greasy, very dark, noncoagulating, tarry appearance, and multiple extravasations will be found in various organs throughout the body.

Spleen:-No swellings or alterations of the spleen have been observed in distemper, except in the rarest cases.

Carcase.-As regards the carcase, one finds it usually emaciated, evil-smelling, and quickly decomposing; rigor mortis sets in as usual, though it is not so marked in septicæmic cases. The skin may be scaly or scabbed from the presence of pustules or bed-sores, the hair is readily detached, and it may be stained yellow if there is any degree of jaundice.

Eyes.-The eyes are sunken in their sockets owing to. the reduction of orbital fat by metabolism, and they are occasionally the seat of more or less diseased processes varying from simple corneal opacities to panophthalmitis (see p. 98). The conjunctiva is sometimes deeply injected; at others it is pale and anæmic, or it may be of a purple hue. Where much ocular discharge has been in evidence, the skin beneath the eyes will probably be found denuded of hair, or covered with crusts, owing to the depilatory action of the discharges.

The following combination of lesions makes a very typical picture of a dog dead of distemper, as revealed on post-mortem examination :

Congestion of the lungs, with areas of hepatisation; inflammation of the fauces and larynx; myocardial petechiæ; congestion of pericardium; acute localised enteritis; slight gastritis; hæmorrhages from mesenteric vessels, spleen and kidneys; slight enlargement of liver and mesenteric glands; congestion of kidneys; much peritoneal blood-stained effusion; no macroscopic brain lesions; cystic congestion and distension. 
This was the result of my most recent autopsy, on a fox-terrier, whose symptoms were: Temperature, $103^{\circ} \mathrm{F}$.; cough slight; moderately increased respirations; little or no conjunctival or nasal discharge; profuse diarrhœa; occasional vomiting; great thirst; no appetite; great dullness; rigors ; and convulsions, followed by coma and death. 


\section{CHAPTER XI \\ DIFFERENTIAL DIAGNOSIS}

To diagnose a case of distemper in its early stages is frequently very difficult for the clinician, unless he is given some history of recent contact-direct or indirect-with a confirmed case of distemper in the neighbourhood. Failing this, he must either await the onset of some one or more of the typical symptoms or judge the case on its merits. For instance, should the animal be a puppy living in or near a town, or be constantly associated with dog-breeders or people attending dog shows, or have recently come from a dogs' home or dog bureau, the practitioner would have reason to be more than usually suspicious of the malady he had to deal with.

I regard it now as an infallible rule (and advise its universal adoption) to treat any of the following deviations from health as distemper, until such time as it can be proved they have no connection with it.

Early Warnings of Distemper.-An unwillingness to play or feed, malaise, shivering, thirst, unusual desire to sleep, and to be as close to warmth as possible, vomiting, an eruption on the hairless parts of the body, slight diarrhœa, indurated mucus in the corner of the eyes, slight huskiness, or the occurrence of fits; any of these conditions, either individually or combined, should lead one to suspect distemper, and (for safety's sake) to isolate the animal, until time has been allowed for the appearance of more pronounced and diagnostic symptoms, or until the primary indisposition has passed entirely away. 
The temperature, unless raised, does not much facilitate a diagnosis, as frequently it remains practically normal for some considerable time, and absence of temperature should not therefore be allowed to dispel one's fears or suspicions.

As it sometimes occurs that a dog may suffer from a very benign or almost unnoticeable attack of distemper, it is a wise plan to continue isolation for a fortnight where practicable, as this not only mitigates the risk of contagion to other dogs from the convalescent one, but also gives time for the appearance of a second and more severe manifestation of symptoms, as often happens.

A diagnosis is arrived at with great facility when several other dogs which have been in contact are noticed to be also ailing; and little doubt need be entertained when once a characteristic group of advanced symptoms has been exhibited. I have known cases in which little or nothing was apparently wrong with the animal except ulcerative keratitis, though this has usually been later followed by other typical symptoms which made the nature of the disease clear.

Dog owners should no longer permit themselves to be lulled into a sense of false security when told their dogs are merely suffering from a chill. They should realise what a'deadly and insidious disease distemper really is, and they would be well advised to regard with the utmost suspicion every sign which is known to be an early warning of this malady.

Diseases confounded zeith Distemper.-There are several diseases which may be mistaken for distemper, among the more prominent being Stuttgart disease (or canine typhus), tuberculosis, epilepsy, rabies, mange, and the many primary catarrhal disorders, such as conjunctivitis, rhinitis, laryngitis, bronchitis, pneumonia, gastritis, enteritis, cystitis, hepatitis and nephritis, etc., all of which 
may arise sporadically from causes distinctly dissociated from those of specific distemper.

Stuttgart Disease.-The outstanding features of Stuitgart disease are the ulceration, necrosis, and gangrene of the edges and free anterior portion of the tongue, the acute gastro-enteritis, and high mortality-60 to 80 per cent. It is comparatively rare to find ulcers of the tongue in distemper, and still rarer to see necrotic or gangrenous lesions, whilst the enteritis is practically always accompanied by pulmonary or some other classical symptoms of distemper. In canine typhus there is always a marked and persistent vomiting (which is frequently absent in distemper), followed in a day or two by extreme lethargy, which in distemper does not usually supervene until a very late stage. The temperature in Stuttgart disease is practically normal throughout; there is no cough and no ocular or nasal discharges; young animals are less susceptible, and when attacked, seem to suffer less severely than old ones, and the disease mostly runs an acute course, death taking place in four or five days.

Tuberculosis. - Tuberculosis may be mistaken for distemper on account of the chronic pneumonia or bronchial catarrh present, combined with a gradually increasing emaciation, and diarrhcea; but tubercle in the dog runs a very prolonged chronic course and there is always a history of slight cough which has been long in existence and is aggravated by exertion. The temperature, too, is irregular - at one time raised, and at another normal-or it may be increased by one or two degrees at night.

Sputum is rarely expelled, as the dog usually swallows it; but its microscopic examination would probably reveal the presence of acid-fast tubercle bacilli.

The diarrhœa of tuberculosis sets in, as a rule, towards the termination of the disease, by which time emaciation 
is so marked that the animal is nothing but a -weak skeleton; there are no ocular or nasal discharges; and none of the typical nervous symptoms of distemper.

Where the investigator fails to demonstrate tubercile bacilli in any discharges or serous fluids, diagnosis máy be attempted by applying the "tuberculin test,". preferably in the morning, and noting the changes of "temperature hourly during the day, and if possible until twenty-four hours have elapsed. The dose of tuberculin for a $\operatorname{dog}$ is $\frac{1}{2}$ to I cubic centimetre injected subcutaneously inside the thigh. The results, however, are not always reliable, and perhaps a better and more convenient method of testing is by the use of ophthalmic tuberculin, wherein from one to two drops of this medium are brought in contact with the conjunctival sac. If the animal is tuberculous a reaction will be manifest in from seven to twelve hours, consisting of the development of conjunctivitis with profuse lachrymation and pus formation.

Epilepsy.-The causes of true epilepsy appear to be so far unknown, and no lesions have been discovered in the brain to account for it. It is believed to be hereditary, and may occur at any period of life. True epilepsy is frequently confounded with the symptomatic variety arising from wasting diseases, and it would be a very difficult matter indeed to differentiate between the epileptiform convulsions associated with distemper and those pertaining to idiopathic epilepsy. The former, however, are usually much more acute and are accompanied by other recognised distemper symptoms, the development of which must be our guide in diagnosis.

Rabies. - The symptoms of cerebral irritation complicating distemper have frequently been mistaken for rabies, and "many dogs have in the past been sacrificed as rabid when suffering: only from epilepsy. 
When a layman sees a dog in the street or elsewhere writhing in a fit or rushing about with a foaming mouth, quite oblivious to all its surroundings or the presence of its master, and, maybe, biting at any object in its path, he might perhaps be excused for concluding the dog is mad; but if the dog has had a fit, this fact alone disproves the presence of rabies, as no epilepsy is ever observed in that disease.

In dumb rabies we notice a deep depression and paralysis of the lower jaw, followed by death in three or four days; in the furious type there is great nervousness and restlessness, snapping of the jaws, and desire to eat hard foreign bodies (depraved appetite) or gnaw chair legs (and human legs if permitted) or other objects. This deliberate aggressive behaviour of rabid animals is, however, absent in distemper; and in rabies there is also a complete absence of any of the various typical symptoms which usually appear in the former malady. Rabies is invariably fatal, death ensuing-after a period of general paralysis-in from three to seven days from the onset of symptoms.

Mange.-When the cutaneous lesions of distemper have become extensive and coalesced, they may present an appearance somewhat resembling that of scabies. Nevertheless, the irritation set up by parasitic mange is decidedly more intense than any produced by the pustular eruption of distemper, nor does mange affect the abdomen and insides of the thighs, except in the rarest instances.

Further, scabies tends to spread gradually and become worse, thus differing from the exanthema of distemper, which, if it spreads at all, does so rapidly, and usually runs its course in about a week, then disappears. In follicular mange we do not find such intense pruritus, but the skin here becomes greatly thickened and corrugated, and 
the disease starts at the head or toes. Since mange may, however, occur simultaneously with a distemper eruption, a diagnosis would be rendered definite upon the search for and demonstration of the causal parasites of mange.

To state precisely whether one is dealing with distemper in its early stages or with a simple non-specific catarrh is a matter of impossibility, since distemper so frequently appears at its onset as a disease of perhaps only one organ, such as bronchitis or rhinitis, etc. The previous history-if any-would have to be taken care. fully into account as before mentioned, after which we would need to await the development of some confirmatory symptom, or microscopically examine the nasal discharge (if any) or a swab from the pharynx or larynx, for the purpose of demonstrating the presence of $B$. bronchisepticus-assuming this organism to be capable of setting up true distemper. 


\section{CHAPTER XII}

\section{TREATMENT AND CONVALESCENCE}

\section{TREATMENT.}

I MAY state at the outset that there is absolutely no specific cure for distemper, as no agent has yet been discovered possessing the property of killing the causal organism of this disease. Thus all the advertisements one reads of "sure remedies for the cure of distemper" must be regarded as calculated deliberately to deceive the public into buying concoctions which can no more perform what is claimed for them than can the bottles in which they are contained.

Quack Remedies.-It is really surprising what faith some members of the community place in these quack nostrums, and withal rather pathetic to think of the poor sick dog being regularly dosed with them, irrespective of whether they are suited to his particular case or harmful. $\mathrm{He}$ is lucky in the possession of the power of voluntary emesis. In view of the diversity of this disease, it must be obvious to all how impossible it is to expect to derive benefit from any one prescription. Each case must be treated on its merits, and since no specific therapeutic agent exists, one must necessarily treat the symptoms as they arise.

All "doggy" persons appear to have their own pet quack remedy by which they swear, yet their dogs continue to die in about the same proportion as ever, and probably very often as a result of the treatment. Gray humorously remarked: "Even at the present time, we have those who believe in the virtue of a "piece of 156 
tarred string' tied round the neck; a 'bent halfpenny'.; a daub of tar on the nose; Christian scientific treatment, and such-like humbug."

Waste of Valuable Time.-It is too true; but the sad part is that after these, doubtless well-meaning; people have tried one remedy after another in their feverish anxiety to find a "cure," they eventually are forced to take their poor animal to a skilled canine practitioner for belated advice and assistance, by which time probably it is nearly dead, or the disease is in such a very advanced stage that little can be done. It only too frequently happens that after all and sundry have tried their skill, and days or weeks have been wasted, the veterinary surgeon is expected to wield some mystic healing power in mitigation of the complaint.

I cannot too strongly condemn the use of quack cureall medicines, most particularly in cases of distemper, as when a practitioner is at last called in to prescribe he has no knowledge of what drug has already been administered, and is somewhat hampered in his choice of medicaments. The simplest prescriptions require some consideration of the animal's temperament, its physical condition, and its breeding, for drugs usually act with a greater precision on well-bred animals than upon those more coarsely bred. Some drugs would be diminished in quantity where an animal is weak or the subject of acute pyrexia; others would be withheld altogether; thus a quack nostrum administered to all and sundry without making a study of the patient is often calculated to do considerable harm.

Mistaken Diagnosis.-Dog owners and others "in the know" frequently attempt to diagnose their dogs' ailments, and as frequently make irreparable blunders, such as mistaking distemper for "worms," then administering vermifuges and purgatives, or other drugs calcu- 
lated to do far more harm than good in such a case. It would thus appear to be a far wiser course, certainly more humane, and probably cheaper in the end, to obtain the services of a skilled veterinary surgeon at the very beginning; one who is competent from his very training and from long experience to give the best advice, and to prescribe the most adequate treatment for each particular case.

Importance of Nursing.-Nursing plays an extremely important part in the successful treatment of distemper; in fact, I often think many cases would progress as favourably without medicinal treatment as with it, provided the hygienic and dietetic conditions were persevered with, and sound in principle. Nevertheless we so often have to contend with such acute disturbances, that very humanity seems to demand that something shall be done to relieve the distressing symptoms.

Hygienic Conditions.-For the purpose of description it will probably be more convenient to deal first with the question of the housing of the patient, for it is not one of the least important factors in our routine treatment. A bright, cheery, and clean environment is very desirable, for when an animal-no less than a human. beingbecomes weak, exhausted, and depressed, it is often the word of encouragement, the cheery glow of its apartment, and the constant little attentions on the part of the nurse, which serve to remind the patient it is still alive, give it some temporarily renewed interest, and for a while help it to forget its pains and weariness-perhaps sufficiently long to bring it safely over the crisis. We know from painful experience, that where patients are left long alone, perhaps in the dark, or in evil surroundings, they lose heart, rapidly sink lower and lower, and die.

Therefore let the room be light, clean, warm, and 
absolutely dry, with all provision made against draughts, yet with an ample exchange of air.

A room is by far the best accommodation for a dog suffering with distemper, as it is thereby allotted a sufficient cubic air space without being actually exposed to external air, and this is important in view of the frequency of lung complications in distemper. It is a popular fallacy that a patient which is the subject of pneumonia should be coddled up in a hot room with all doors and windows closed; but never was there a greater mistake, for the more frequently fresh air can be substituted for used air the better, commensurate, of course, with the maintenance of an equable temperature and absence of draughts or swiftly moving currents.

Litter.-Any litter may be strewn upon the floor for the purpose of absorbing urine and discharges, though wood-wool or wood chips are probably far better than sawdust, owing to the dusty, and therefore irritant, properties of the latter. There is a certain amount of antiseptic value in wood as a litter, on account of the resins and volatile oils contained. Further, when soiled it should, and can easily, be burnt. This material serves equally well for bedding, and is preferable to blankets or cushions, for whilst affording the necessary warmth, it does not harbour parasites and may be replaced cheaply when soiled. It is desirable that the box or other receptacle in which the dog is to lie should be slightly raised from the floor, as a current of cold air frequently passes along the latter from the bottom of the door to the chimney. Rather than the box should be made wet by disinfecting, it should be replaced occasionally by a new one, thereby helping to rid the room of much of the unpleasant odour associated with cases of distemper.

Room Temperature.-The temperature of the room 
should not be too high, and the optimum will be found at about $60^{\circ} \mathrm{F}$. It is a mistake to overheat the sick-room, as this only tends to make the patient more than ever susceptible to cold, and, in any case, is oppressive. It is equally bad practice to allow the fire to go out upon the owner retiring at night, or-to extinguish the gas or other heating appliance, as the early hours of the morn-

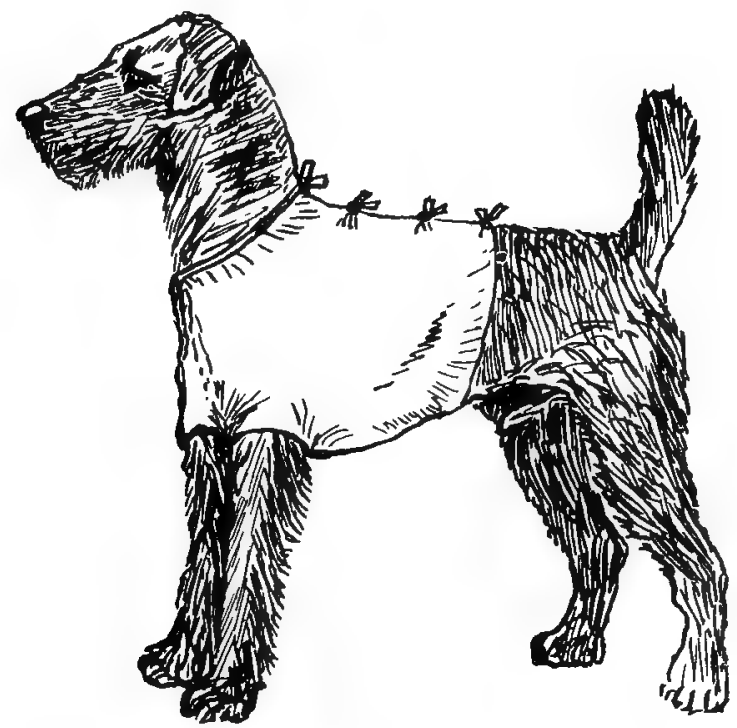

Fig. i8.-Coat for Chest Protection in Lung Complications.

ing are the coldest, and alternations of temperature are harmful.

Clothing.-Generally I do not favour the application of clothing to dogs if the above conditions can be complied with; but, if not, it might be deemed advisable to make a little jacket out of some soft, dry material, which would at least protect the chest from possible chill during inclement weather. In order that such a jacket should be comfortable and efficient, two holes should be cut near the front edge of a square piece of material, through 
which the front legs should be passed, the sides of the material being brought up over the back and fastened with safety-pins; the front edges of the material should be joined with safety-pins, or stitched together over the breast, due care being taken to provide that it shall not be tight or tend to impede deglutition or respiration. (See Fig. 18.)

Where counter-irritants have been applied to the chest for the relief of pulmonary symptoms, such a jacket will become essential, and, for the same purpose, Thermogene

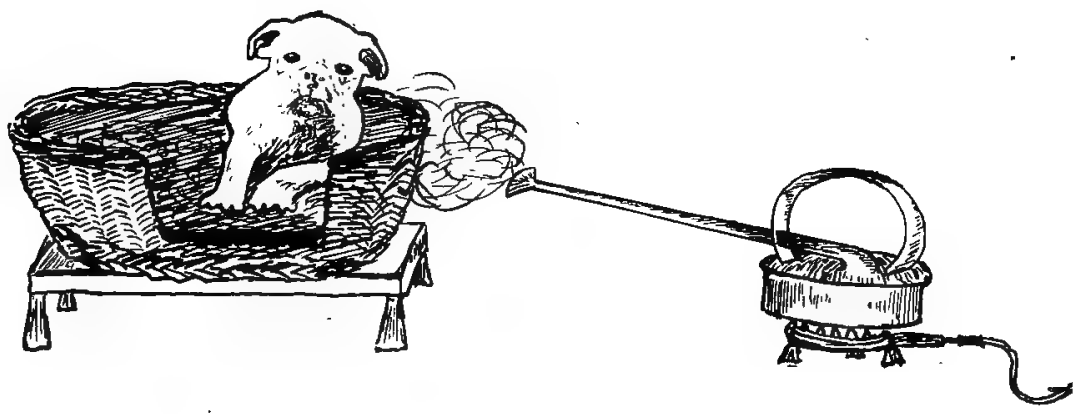

Fig. ig.-Inhalation of Steam. Arrangement of apparatus.

wool can usefully be interposed between the chest walls and the clothing.

Steam.Kettle.-If dyspnœa is marked, much relief can be afforded by the use of a steam kettle arranged in such a manner that its vapour will arise in the vicinity of the dog's head. To a pint of water, a few drops of eucalyptus oil, tincture of benzoin, Sanitas, Jeyes' Fluid, or oil of turpentine, may be added, which, when the water boils, will charge the atmosphere with a pleasant antiseptic inhalation having decided curative properties in the catarrhal conditions of the respiratory organs.

Removal of Discharges.-Great attention must be paid to the cleansing away of all discharges, particularly from 
the eyes, as, if this be not done at very frequent intervals, the eyelids become gummed together and the eyelashes stuck fast in the dried exudate which forms so rapidly in these cases, the eyes being not only rendered difficult to open, but serious complications to the eyeball being induced from the continued presence of irritating pus. Few conditions are so depressing to the spirits as a painful affection of that most tender of all organs, the eye, and, if only in consideration of the animal's suffering, we must at least take pains to remove all purulent accumulations, bathe and soothe the parts with warm antiseptic solutions (particularly hydrogen peroxide), dry them, and then anoint the edges of the lids with a suitable ointment which will asepticise and prevent sticking. (See under "Eyes," p. I77.)

Similarly the nostrils must be wiped at the same time, to prevent occlusion and obstruction to respiration, for the nasal discharge tends in like manner to accumulate around the nasal orifices, dry into hard scabs, and cause ulceration or cracking of the skin and mucous membranes. These operations should be carried out with pledgets of cotton-wool, soaked in weak warm solutions of boracic acid, Condy's Fluid, Jeyes' Fluid, or Sanitas : but for the eyes nothing stronger should be employed than physiological salt solution, boric acid (saturated solution), or zinc sulphate ( 1 per cent. solution.)

Each pledget should be placed in a china or metal receptacle until the cleansing is complete, when all must be burnt.

Body Cleanliness.-Fæcal or urinary stains about the hinder parts of the body should also frequently be washed off, and careful attention paid to the under parts of the tail and around the anus, for it is often found in distemper that a dog will pass its stools as it lies in its bed, being too weak or too disinclined to move; and in 
long-haired varieties the hairs are sometimes found imbedded in the fæcal mass, which can only then be removed by cutting away all attached hair with scissors. So little attention is paid to this important and elementary matter that I feel bound to call attention to it. It has been no uncommon occurrence to have dogs brought to my surgery in this condition-often with the history that the dog is constipated and in great pain, when the sole trouble has proved to be neglect on the part of the owner, leading to a purely mechanical obstruction to defæcation. In any case of debilitating disease where the subject is of the long-haired variety, it is a sound precaution to clip away all hair from the vicinity of the anus, prevention in such instances being better than cure.

The ears should be periodically examined, as occasionally otorrhoea is set up, the discharge of which would need constant removal and the application of medicaments.

Cleansing the Mouth and Teeth.-Where the patient has an evil-smelling mouth, dirty teeth, or inflamed buccal mucous membrane, the nurse must also devote a little time to this condition by the application of mouthwashes, consisting of solutions of permanganate of potash (I to I,000), of Sanitas (I to 4 or 5), or hydrogen peroxide ( $\mathrm{I}$ to 4). A very nice mouth-wash, known as Glyco-Thymolin, can be purchased from any chemist in tablet form, one tablet being dissolved in water for edch application. A solution of boracic acid is sometimes used, but it is a very mild antiseptic, and I prefer the agents previously mentioned. Cleansing of the mouth and teeth may be carried out with a small sponge attached to the end of a stick or held with forceps, or a soft toothbrush may be employed.

Exercise.-Differences of opinion exist as to the advisa- 
bility of allowing a dog sick with distemper to take exercise. Generally speaking, it should be prohibited, except in the most restricted amounts, no matter whether the disease is in its inception or well advanced, for the reason that it excites and fatigues, elevates the temperature, and imposes a greater burden upon the heart in having to supply extra blood to the muscles of locomotion. On the other hand, gentle exercise increases natural peristalsis and encourages the action of eliminatives (where these are required), and prevents to some extent the leg weakness which so often arises from continued lack of movement. Rickets has also been cited as a sequel to the withholding of exercise, especially in heavy puppies, which then become perhaps permanently disfigured.

Thus I am in favour of allowing the patient the run of its room, but not of the house or yard, and by no means should the patient be taken for walks, however bright he may appear. In the case of house-trained dogs, which refuse to evacuate except out of doors; a large box containing a layer of ordinary earth should be provided, and the animal induced to use it. At the termination of the attack, convalescence should be well advanced before normal exercise is resumed, and then it should only be allowed for small, gradually-increasing periods, the dog to be obviously quite strong before the open street is faced.

Failure to observe these precautions has led in numerous instances to relapses or nervous complications, such as fits or chorea.

Minimising Spread of Contagion. - In order to minimise the spread of contagion from the sick-room to other dogs and places by the attendant treading on nasal or coughed-up discharges or fæcal matter, the floor of the room may be sprinkled with a o per cent. solution of cresol (Jeyes' fluid) at intervals during the day, or with 
Sanitas powder. Neither is toxic or causes irritation to skin or mucous membrane, but both are anti-parasitic, and respiratory antiseptics. In this connection isolation of the sick dog is of little use in curtailing the spread of infection to other dogs if the person who attends the animal is not also isolated, for the contagion is readily carried on his clothes, boots, and hands unless overalls and goloshes are worn and the greatest precautions observed.

Excrement should be covered and surrounded with pine sawdust or kennel powder as soon as noticed, as this facilitates its removal, and helps one to avoid it prior to such removal.

In those instances where more dogs than one are affected with distemper, and are being treated under the same roof, it is a good precautionary plan to isolate each from the other as much as possible, particularly if the infection has not originated from the same source, because we may find one dog has fallen to a comparatively virulent attack, whilst its companion has contracted only a mild type, and it is quite conceivable that the former will transmit its malignant contagium to the latter, producing in it severe symptoms or complications which might never have arisen had the individuals been kept epart.

Stimulation of the skin by gentle brushing each morning is attended by good results in all cases except those which have become too weak to withstand the fatigue of being handled; therefore the activities of the nurse in this direction must be guided by the indications of the patient's condition. The brushing of a dog's coat has exactly the same stimulating and refreshing effect on the $\operatorname{dog}$ as the morning wash has on the human being.

Dieteties.-The proper dieting of a sick dog is of paramount importance, and its methodical execution is a 
duty which devolves upon the nurse no less than the observance of all the hygienic principles enumerated above, and unless the person undertaking the duties of nurse fully realises his or her responsibilities, and carries them out to the letter, the patient stands a poor chance of recovery, for no amount of medicinal treatment can ever compensate for lack of efficiency in nursing and feeding. Our patients cannot be reasoned with; thus, when the appetite is completely in abeyance, forcible feeding becomes necessary, and the important point is to know how to do it without upsetting or exciting the animal. It may be regarded as a fundamental rule in the feeding of sick animals that food shall be offered only in very small quantity each time, but at much more frequent intervals ; for in this way the overtaxing of the digestive organs is avoided, and only as much nourishment is consumed as can be assimilated.

In the early stages of distemper the appetite very often remains remarkably good-even in spite of a temperature-and in such cases food should not be withheld in consequence of the fever, although the meat ration and other nitrogenous substances may be reduced.

A light nourishing diet is indicated, such as is afforded by milk, bread and milk, or boiled fish, etc. Should there be no pyrexia at the commencement, I advocate the continuance of the meat ration so long as the dog will eat it voluntarily, for meat must be regarded as the natural and normal food of the canine race. Milk is an ideal diet for the sick, and can in most cases be quite easily digested and retained; and in addition it contains all the tissue-building vital elements necessary for the sustenance of the system. If the dog has lost all inclination for food it is hardly wise to commence its forcible administration immediately; better far to coax the animal by offering little pieces of food out of the hand, and by 
tempting him with a variety of dishes, preparing one's self to be satisfied at first with the small amounts voluntarily taken, for food which is voluntarily eaten is worth a good deal more than that which is forced down.

Of course, refusal to feed cannot be countenanced indefinitely, as a dog will very soon lose strength and condition if not nourished; therefore, having allowed two or three days to pass during which only the slightest morsels of food have been consumed, and the alimentary tract having had a perfect rest, we must then proceed to administer artificially such aliment as appears indicated.

Sick Diet.-Probably in the first instance pure milk will suffice or a new-laid egg may be beaten up in it; to this a little port wine may be added for cases which seem to require an alcoholic stimulant. Beef-tea, soups,

- broth with the yolk of an egg in it, extract of meat, small quantities of minced raw meat, are all very good diets for sick animals such as those suffering with distemper, and for the sake of variety they may be given alternately. There are some proprietary meat extracts which, owing to an excessive content of sodium salts, may sometimes increase the irritability of the intestinal mucous membrane, and aceentuate any already-existing diarrhœa. These should be avoided if their consumption is noticed to be followed by ill-effects.

Whey is very good in cases of weak digestion, gastritis, etc., and is made by heating a pint of milk to about the temperature of the body, and adding a teaspoonful of essence of rennet. Break up the curd and strain off the whey through muslin.

Raw meat juice can be prepared by finely mincing some steak and placing in a jar containing cold water in the proportion of one part of water to four of meat. Let it soak for half an hour, then press out the juice through muslin. 
To make beef tea, cut some lean beef into shreds; place in a saucepan with cold water, and let it cook very slowly for three to four hours; add a little salt; skim off the fat and strain. It must never be allowed to boil.

1 have noted on several occasions during protracted illnesses that an animal will evince a sudden desire to feed voluntarily, and will consume solids almost ravenously if permitted; yet in such cases it is obviously undesirable to impose such a severe burden on digestive organs which have probably been for so long accustomed only to the lightest diet in carefully regulated quantities, and the resumption of feeding with solids must, therefore, be undertaken on a strictly sliding scale and with great caution.

Foods for Diarrhoea.-Where there is a profuse diarrhœea which it is highly desirable to check, this can be often better accomplished by food than by medicine, and for the purpose some astringents such as cornflour, arrowroot, or rice starch should be mixed with milk and egg, and administered in small quantities at frequent intervals. Arrowroot is particularly soothing and nourishing, and forms a valuable adjunct to the sick diet. Milk puddings, such as sago, rice, or semolina, are also useful if the dog has sufficient appetite to eat them. A food I have frequently used in severe cases with apparent benefit is a mixture of one egg, half a pint of milk, half an ounce of port wine, and cornflour to thicken, administered four times daily.

Aids to Retention of Food.-If the swallowing of liquid or other foods is followed by emesis, we must try the effect of giving less at a time until this evil abates; milk may be mixed with soda-water, or with ice, which sometimes act as effective antemetics, but where all such efforts seem abortive, we must then resort to the treatment of the stomach with drugs, among the best of which 
for the purpose is bismuth. This agent, in virtue of a mechanical action by which it is slowly deposited upon the walls of the stomach, allays irritation thereof through more or less prevention of contact between the gastric mucous membrane and the foods ingested. It is also a germicide of considerable value in bowel cases, the benzoate or subnitrate being mostly employed. These can be administered in powder form on the back of the tongue in doses of from 3 to 20 grains, three or four times daily, or may be suspended in some mucilage. A very convenient mixture is that known as mist. bismuthi co. (Hewlett), containing in each fluid drachm:

$\begin{array}{lllll}\text { B. Bismuth. carb. } & \ldots & \ldots & \ldots & \text { grs. v. } \\ \text { Liq. morphinæ } & \ldots & \ldots & \ldots & \text { m iiss. } \\ \text { Sodii bicarb. } & \ldots & \ldots & \ldots & \text { gr. iiss. } \\ \text { Mucilage } \quad \ldots & \ldots & \ldots & \ldots & \text { q.s. }\end{array}$

the dose of which ranges from 3ss. to zii. according to the size of the dog.

Hydrocyanic acid in doses of $m i$. to $m i v$. of the dilute acid is a powerful antemetic, as also is chloretone. The former acts by its paralysant effect on all nervous structures with which it comes in contact; the latter exerts a nerve sedative and antispasmodic action, and is the active principle employed in most of the well-known proprietary "sea-sick remedies." It is non-irritating, non-toxic, and has no action on the heart, and as a remedy for gastritis and vomiting in dogs it is invaluable. One or two of the 5-grain capsules are usually sufficient to arrest emesis. If the capsules themselves act as irritants, and are thrown up before they have had an opportunity of dissolving, the chloretone must be given in liquid form, the contents of a capsule being dissolved in half a teaspoonful of brandy, and a teaspoonful of warm water then added.

Hot fomentations to or stimulation of the abdomen will 
also help to relieve the symptom, and concluding that by these various means we have brought the patient into a condition which enables it to swallow and retain liquid foods, the question of the selection. of these foods may be resumed.

Of the proprietary foods there is an extensive choice, and of them all probably Virol has the most value, containing as it does bone marrow and malt extract. Brand's essence of beef is also very excellent, and as a food for dogs, should be warmed until liquefied, then given with a spoon. Benger's, Allenbury's, and many patent proteid foods-such as those containing phosphates-are beneficial and reliable, and some splendid results have accrued in nervous cases from the use of Sanatogen.

Rectal Feeding.-It may happen, of course, that in some cases the administration of food by the mouth becomes impossible or undesirable, owing to excessive weakness, sore throat or mouth, poor digestion, or vomiting, when recourse must be had to rectal feeding.

This may be accomplished in two ways, viz.: (1) Clysters; (2) Suppositories.

Giving a Clyster.-In giving a clyster the rectum should be first washed free of fœild mucus or fæces by the injection of warm saline solution (I teaspoonful to the pint) with an apparatus constructed as follows: A length of rubber tubing-about 2 or 3 feet-with a lumen of $\frac{1}{4}$ inch, is attached at one end to a rubber (human) rectal tube by a glass connection; at the other end a glass funnel is inserted. The rectal tube is anointed with vaseline, the solution is poured into the funnel until it runs out at the lower end, when the tube can then be carefully inserted as high as possible into the rectum. It is wise to hold the funnel at only a slightly higher level than the rectal tube until the latter is introduced, and the object of allowing the water to run before insertion is to exclude all air. 
Whilst the tube and funnel are still full, some of the liquid having already passed into the bowel, the funnel must be quickly lowered and inverted over a pail or bowl; then by the simple act of siphonage all the fluid in the rectum will run out. The funnel is now raised again and

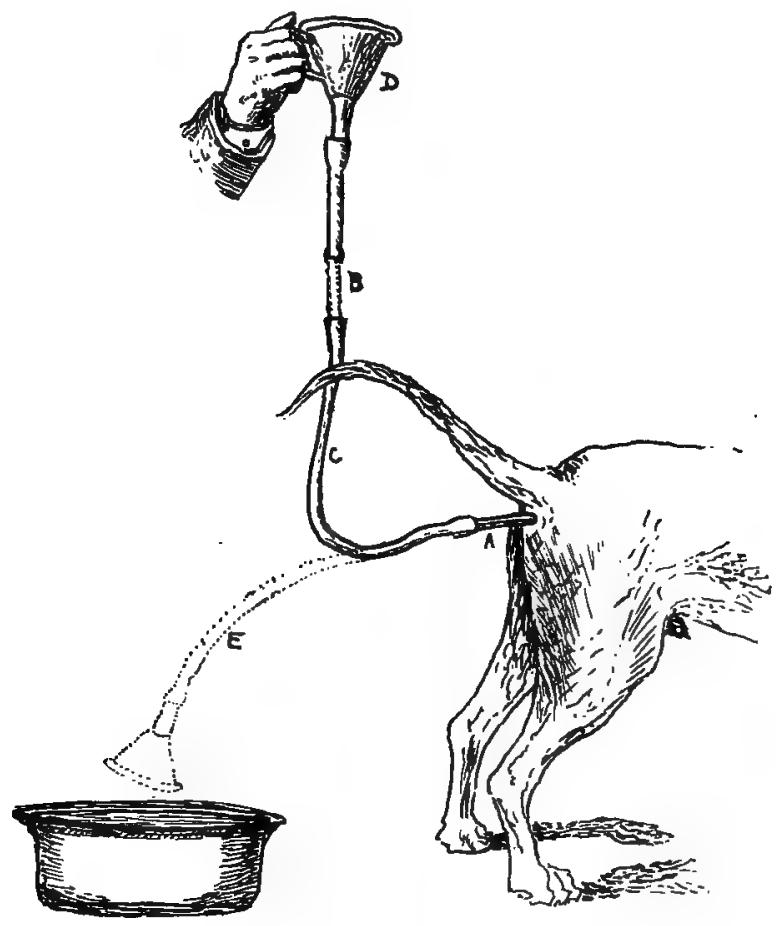

Fig. 20.-Rectal Lavage.

The upper position of the funnel is that used for pouring water in, whilst the lower position allows it to run out.

$A$, rectal tube ; $B$, glass connection ; C, rubber tube ; $D$, funnel ; $\mathrm{E}$, the apparatus inverted.

(Modified from "Miller and Glass.")

the operation repeated, without, of course, removing the tube from the rectum. The rectal tube used for this purpose has a terminal hole of good size diameter. If any air passes in, great pain is often occasioned.

Ten minutes after the washing process the nutritive 
clyster may be injected in like manner, though, of course, none is syphoned out, and in this operation a long rubber catheter (as used on human beings) or an ordinary mare's catheter, with a lateral instead of a terminal orifice, is substituted for the rectal tube; and, as before, it must be greased prior to introduction and passed high up in a gentle manner. The liquid must only be allowed to enţer at low pressure, to accomplish which the funnel must only be slightly elevated; the lower the funnel is held the slower will the food enter, and the greater probability will there be of its retention and absorption.

Fig. 21,-Soft, Pliable Catheter for Rectal Use in giving Nutrifint Enemas (With Lateral Orifice).
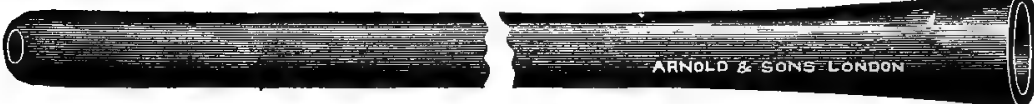

Fig. 2ia.-Rubber Rectal Tube for Rectal Lavage (with Large Terminal Hole).

The material injected will, of course, be at blood heat -i.e. about $100^{\circ} \mathrm{F}$. Upon withdrawal of the catheter the anal opening should be kept closed, and it is an advantage to slightly elevate the dog's hind-quarters for awhile, if such can be done without causing undue excitement. Clysters are sometimes returned unless great care is exercised, and the points to be noted are :

I. The liquid must be at blood heat, so that its introduction will not be felt.

2. No air must be injected.

3. The tube must be greased and carefully introduced.

4. Injection must be very slowly performed, and not in greater quantity than one to two ounces at a time.

Composition of Nutrient Enemas. - The enema may con- 
sist of meat broth, egg and milk, or broth and egg, and to give it sufficient consistence, thick starch water may be added. In cases of great emáciation and prostration such nourishment should be given every two or three hours. It is surprising how long an animal may be kept alive by this means, and very frequently it has proved the deciding factor between death or recovery.

Albrecht recorded an instance of life being sustained for forty-two days by this method.

Nutrient Suppositories.-Nutrient suppositories can be purchased in boxes of twelve, each suppository containing nourishment representing half an ounce of almost entirely peptonised beef. They are biconical in shape, and need only to be moistened before insertion, and then passed as high as possible by inserting the rectal tube behind them. Previous rectal lavage, however, is also here indicated, especially if the bowels are in an unhealthy, dirty state.

History Sheets.-In some veterinary infirmaries, where great attention is paid to detail, history sheets are kept, upon which the condition of each case is entered as it is admitted, subsequent particulars being noted from day to day. Reference to such a chart will readily indicate any progression or retrogression made, and is not only instructive to the surgeon and his assistants, but is also evidence to the client of the scrupulous care which is being bestowed on the sick animal. A useful form of chart will be found on p. I74.

Medicinal Treatment-Early Stages.-When the practitioner's attention is called to a case in its early inception, he may adopt one of several courses-viz. : (I) He may give an emetic, such as vin. ipecacuanhæ (3ii. to $3 \mathrm{v}$.) per os, or apomorphine (gr. $\frac{1}{17}$ to $\mathrm{gr}, \frac{1}{2}$ ) subcutaneously. Finlay Dunn says: "By clearing out both the stomach and biliary system, emetics remove biliousness, 
and used at the outset they thus mitigate distemper and other febrile attacks, and sometimes arrest epileptic seizures." Nevertheless, they also produce nausea and depression, and it is doubtful whether they are not on that account even more harmful than beneficial, and personally I never employ them in cases of distemper.

(2) If diarrhœa is not already in evidence, he may administer calomel in doses of gr. $\frac{1}{5}$ to grs. ii. once or twice daily until a laxative effect is produced. Friedberger and Frohner carried out some experiments on cases in which the digestive tract was the starting-point of the infection, and they stated that good results were obtained from giving gr. $\frac{3}{4}$ two or three times a day, pointing out its disinfectant action on the mucous membranes of the stomach and intestine, and the probability of thereby preventing complications. There is no doubt that the emptying of the stomach and bowels has a salutary effect, and if constipation is present, a laxative seems essential.

We must, however, always bear in mind the importance of refraining from reducing the strength and condition of the animal, since we may later be hard pressed to sustain either. Probably our object would be achieved just as satisfactorily if a warm soapy enema were given instead of purgatives, or a glycerine suppository tried.

Fever.-(3) If no other symptom than dullness and slight elevation of temperature are observed, he may employ febrifuges, such as quinine sulph., aspirin, sodium salicylate, antipyrine, spt. æther. nit., etc. In prescribing quinine one must not lose sight of the fact that large doses sometimes cause nausea and vomiting, also weaken cardiac action and reduce blood pressure, whereas small doses are stimulating; let the dose therefore not exceed gr. $\frac{1}{2}$ to grs. iii. according to the size of the dog. Aspirin may be administered in powder form 
in quantities of grs. v. to grs. xv. three times daily; it is a fairly safe drug, very large doses being required to set up toxic effects, these being of a paralytic nature.

However, it is questionable whether antipyresis is justifiable when elevation of temperature is only slight or of short duration, for, as Müller correctly observed, it deprives us of the symptom of temperature, which is of the greatest importance during the course of the disease, and, moreover, many of the agents used are cardiac depressants.

It is only when pyrexia has been long persistent or has attained an alarming height, thus threatening to induce some deleterious effect upon the vital organsparticularly the heart-that interference becomes of paramount importance.

Antipyrine is a first-rate febrifuge for dogs, producing its effect within half an hour, without any untoward action, and it may be given in doses ranging between grs. v. and grs. xv. frequently during the day.

Antifebrine is another quick-acting drug of great value for rapidly lowering temperature in cases of fever arising from pneumonia or any other cause; it is cheap and safe, and is usually administered in doses of grs. ii. to grs. $\mathrm{x}$. every two or three hours.

Both of these agents are quicker in action, more certain, and preferable to quinine as antipyretics, and deserve to be more widely used in canine practice than they have been hitherto.

Other remedies for fever are the various salines, such as magnes. sulph. or Glauber's salt (a pinch of either of which could with advantage be dissolved in the drinking water); warm clothing to the body, but cool, fresh air to breathe; rest, and a regular action of the bowels.

Apart from these measures, and advice as to isolation and segregation of the ailing and in-contact animals, with 
especial reference to the hygienic and dietetic treatment of the patient, nothing more need be attempted at this stage, and unless complications arise, probably nothing more will be required. In the reverse case, other therapeutic measures must be adopted as the symptoms arise, for the treatment of distemper is practically entirely symptomatic.

Ocular Lesions.-Attention must be paid to eye discharges from the very beginning, for the reasons already stated in the chapter dealing with symptoms of the eye. (See also p. I6 .)

Conjunctivitis.-Where conjunctivitis is present, a solution composed as under and applied three times per day is very effective; in fact, I have found no eye lotion to equal it in most cases:

\section{PRESCRIPTION NO. I.}

$\begin{array}{llcccc}\text { B. Zinci sulphatis } & \ldots & \ldots & \ldots & \ldots & \text { gr. ii. } \\ \text { Ac. boric. } & \ldots & \ldots & \ldots & \ldots & \text { gr. } x . \\ \text { Aquæ dest. } & \ldots & \ldots & \ldots & \ldots & \text { ₹. }\end{array}$

Boracic lotion ( 5 to ro per cent.) may be used alone; and in all instances where aqueous solutions are employed on the eye these should always be warmed before application, as the dog does not then resent the operation so mutich, nor is the eye so sensitive to a warm solution as to a cold one.

If the secretions are purulent and the inflammation of the conjunctiva is intense and obstinate in character, it is often advisable to paint the membrane with a solution of nitrate of silver ( 1 to 80 ) once daily for several days, followed shortly afterwards by a washing with a 2 per cent. solution of common salt to neutralise the irritant action of the silver salt. Various other remedies may usefully be employed, such as solutions of copper sulphate ( 2 per cent.), corrosive sublimate (0:02 per cent.), creolin 
( $\frac{1}{2}$ to I per cent.), permanganate of potash (0.05 per cent.), and salicylic acid (I per cent.).

Dry calomel or tannoform blown or dropped on the eyeball in small quantities is favoured by some practitioners.

In all eye cases which are characterised by marked photophobia and sensitiveness, 2 drachms of liq. opii sed. may be added to each ounce of the zinc or boric lotions mentioned above, and the combination will be found a very excellent sedative and astringent eye-wash.

If the pain is intense, $m v$. of a 2 to 5 per cent. solution of cocaine hydrochlor., dropped into the eye, will produce complete insensitiveness in a few minutes, lasting, however, not much more than ten minutes. It may be repeated as required, or cocaine ointment could be smeared on the eyelids at frequent intervals.

Unless severe ocular irritation is relieved, we often find the dog scratching at its eyes or rubbing them at every opportunity, with the result that traumatic ulcers or wounds are not infrequently produced. To obviate such a contingency, shields of various kinds have been devised, but unless they are perfect in fit and comfortable to wear, they usually cause more harm than good, and worry the dog incessantly. Subdued light is , an advantage where photophobia exists, and if the animal can be kept in a darkened room while the eye trouble is at its height, no shield or bandage will be found necessary.

Keratitis.-For the treatment of parenchymatous keratitis frequent bathing with warm antiseptic solutions will materially contribute to the reduction of inflammation, and other treatment may be prescribed as for conjunctivitis, whilst, in addition, a half per cent. solution of atropine sulph. is very useful.

Blenorrhoea.-In obstinate cases of blenorrhoea, cautery 
with pure silver nitrate or copper sulphate, or even actual cautery, has been recommended, although this must only be undertaken during the local anæsthesia produced by previous application of cocaine. Other minor operations can be performed under the same conditions, such as removal of the membrana nictitans, or its treatment in obstinate catarrhal conjunctivitis.

Ulcerative Keratitis.-Ulceration of the cornea usually yields to treatment with the zinc and boric lotion; but, should it not do so, hot fomentations containing 5 per cent. boric acid should be tried every four hours, and if they are successful they are much preferable to cautery. Lotions containing lead must never be used in cases of injury to the cornea, as lead salts are liable to be permanently deposited in the substance of the membrane. If there is hernia of Descemet's membrane with threatened rupture, it is advisable to apply a few drops of eserine solution to the eye (0.05 eserine salicylate to 10.0 of distilled water), as this reduces intra-ocular pressure and is probably a safer proceeding than that of puncturing the membrane at its lower border. The object aimed at is, of course, reduction of the pressure of liquids in the anterior chamber, and if this can be effected by local applications, the actual puncture of the chamber would appear contraindicated, even though carried out below the line of vision.

Atropine, although advocated by some in this condition, possesses the property of dilating the pupil and increasing intra-ocular pressure, therefore is hardly a suitable remedy. Other treatment for ulceration consists in dusting the eye with calomel, or the use of the various other lotions already enumerated. "Oculets," containing $\frac{1}{200} \mathrm{gr}$. of adrenalin, are very valuable in inflammatory conditions of the eye, as they quickly reduce hyperæmia. Where this type of ophthalmic medication is preferred 
to collyria an assortment of these Oculets can be obtained, and their application is effected by placing one into the conjunctival sac by drawing forward the lower eyelid.

Old-Standing Opacities.-The removal of old-standing opacities may be hastened by smearing the eyelids with red precipitate ointment ( $\mathrm{I}$ to 20 ) or yellow oxide of mercury ointment, night and morning. The latter remedy has been found especially efficacious against indolent ulcers and blemishes of the eye; and in addition its presence materially helps to prevent the sticking together of the eyelids so commonly seen in distemper, and the excoriation of eyelids and skin adjacent to the eye. Müller obtained far better results from calomel than from anything else for the eradication of sclerotic spots; he used it in equal proportion with grape sugar and placed a small quantity of the mixture on the cornea daily, and massaged the eyelid for ten minutes. The same author mentions the fact that permanent opacities may, if necessary, be disguised or modified by tattooing. The eye is cocained and the punctures made in an oblique direction in order to avoid complete puncture of the cornea. Sepia or Indian ink solutions are then rubbed into the punctures by massaging the lid over them for ten or fifteen minutes at a time.

Cataract and Staphyloma.-The treatment of grey cataract and staphyloma is surgical, the technique of which I do not propose to consider in this work. Therefore I must request practitioners and students to refer to the many publications which describe these operations in detail.

Respiratory Disorders-Nasal Catarrh-The nostrils are frequently the seat of muco-purulent or purulent discharges, which should be carefully removed with moist antiseptic swabs, especially when the secretions 
tend to occlude the nasal passages and impede easy respiration. To prevent excoriation of adjacent tissues the nose should be anointed with boracic or zinc ointment or vaseline.

All swabs used for this purpose must be burned, as nasal discharge is very infective. Inhalations of medicated steam are very useful for disinfection of the respiratory mucous membranes, and for the purpose we can hardly find a better agent than creolin (Jeyes' Fluid), I to 2 per cent. ; others have been referred to on p. I6 1 .

If the nasal catarrh is very acute, the nostrils may be sprayed with an atomiser, using any of these preparations or an infusion of camomile.

Laryngitis.-Catarrh often extends to the larynx, the condition being a precursor of distemper, and manifested by a hard, dry, and often loud cough, with pain or tenderness in the region of the glottis. Soothing expectorant mixtures are prescribed for the alleviation of this symptom, such as :

\section{PRESCRIPTION No, 2.}

B. Liq. morph. hydrochlor. ...

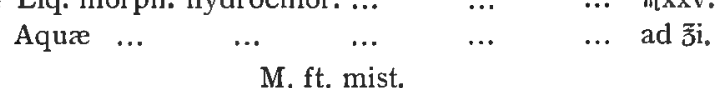

Sig. : One teaspoonful every four hours.

PRESCRIPTION No. 3 .

Bx Mist. heroin co. conc. (Hewlett) ... ... 3ii. Aquæ ... M. ft. mist.

Sig. : One teaspoonful to be given every three or four hours. PRESCRIPTION No. 4 .

R. Tinct. belladonn. Spt. camphoræ ... Glycerini

$$
\begin{array}{llll}
\ldots & \ldots & \ldots & \operatorname{mxl} . \\
\ldots & \ldots & \ldots & 3 \text { ii. } \\
\ldots & \ldots & \ldots & \text { ad } 3 i .
\end{array}
$$

Mi. ft. mist.

Sig. : One teaspoonful to be given every three or four hours. 


\section{PRESCRIPTION No. 5.}

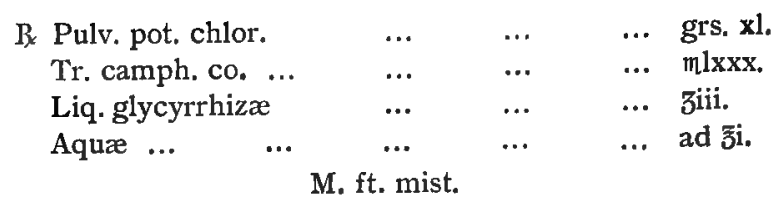

Sig : One teaspoonful to be given every three hours.

Please Note.-The doses recommended in all these, and subsequent, prescriptions are calculated as for the fox-terrier or similar dog twelve months of age.

External treatment consists in the application of hot fomentations or poultices to the throat, or the use of counter-irritants-such as soap liniment, ordinary embrocation, cantharides ointment, or mustard. Fomentations should never be used in conjunction or alternately with rubefacients, as a blistering effect is then produced which may be undesirable.

Bronchitis.-For bronchitis, morphine and codeine are both very efficacious, although better than either is the concentrated mist. heroin co. The principal advantages of heroin over morphine or codeine are that, whilst its dose as a sedative is much smaller, it does not cause constipation, and the blood pressure is not altered in any way. It possesses the property of lowering the temperature to a greater degree than morphine, whilst not giving rise to the cramp-producing effect which codeine exerts if given in large doses. Heroin is not more toxic than codeine, whilst the dose is smaller and the effect greater in all cases of laryngitis, bronchitis, or pneumonia.

An efficient soothing and expectorant combination, especially suitable for dogs, may be found in Cocillana Compound Syrup (Parke, Davis), which is particularly adapted to the treatment of acute or chronic bronchitis when the secretion is scanty and cough excessive. The dose is 3ss. to 3ii. in water. 
A well-tried prescription in my own practice is :

\section{PRESCRIPTION No. 6.}

$\begin{array}{lcccll}\text { R. Spt. æth. nit. } & \ldots & \ldots & \ldots & \ldots & \text { 3iv. } \\ \text { Spt. amm. arom. } & \ldots & \ldots & \ldots & \text { 3iiss. } \\ \text { Vin. ipecac. } & \ldots & \ldots & \ldots & \ldots & \text { 3ii. } \\ \text { Tr. camph. co. } & \ldots & \ldots & \ldots & \ldots & \text { 3i. } \\ \text { Aquæ ... } & \ldots & \ldots & \ldots & \ldots & \text { ad oii. } \\ & & \text { M. ft. mist. } & & & \end{array}$

Sig. : Two teaspoonfuls four times daily.

To this may be usefully added mlxiv. of chlorodyne if any diarrhœa is present, or liq. glycyrrhizæ (q.s.) if the dog is constipated.

The animal should be kept in an equable temperature of about $60^{\circ} \mathrm{F}$., protected from draughts and damp, though in a well-ventilated atmosphere. Hot drinkssuch as hot milk, broths, soups-are soothing and beneficial. In cases where the cough is very severe and continuous, the dog becomes much exhausted and de. pressed, and the administration of expectorants is a necessity to reduce the accumulations of mucus.

I am a great believer in medicated inhalations of steam for all respiratory ailments, and although some writers belittle their value, they can at least do no harm if no good, and should be tried.

Where there is evidence of heart weakness, give stimulants such as liq. ammon. acet. (3i. to 3iii.), spt. æth. nit. ( $3 \frac{1}{4}$ to 3 ii.), tr. digitalis ( $m v$. to $\left.m x x.\right)$, spt. camphor. ( $m$ vii. to $m \times x \times$.), brandy (ro to 30 drops). Syrup of squill is also a good heart tonic, expectorant, and diuretic, of which dogs take $m \times$. to $m \times x x$., usually in conjunction with digitalis or ammonium acetate solution.

A beneficial recipe for dogs the size of a fox-terrier would therefore consist of : 
PRESCRIPTION NO. 7 .

B. Liq. ammon. acet. Tinct. digitalis ...

Syr. scilla

Glycerini

Aquæ ...

$$
\begin{array}{lll}
\cdots & \ldots & \ldots \\
\cdots & \ldots & \ldots \\
\cdots & \ldots & \ldots
\end{array}
$$

M. ft. mist.

Sig. : Give two teaspoonfuls three or four times daily.

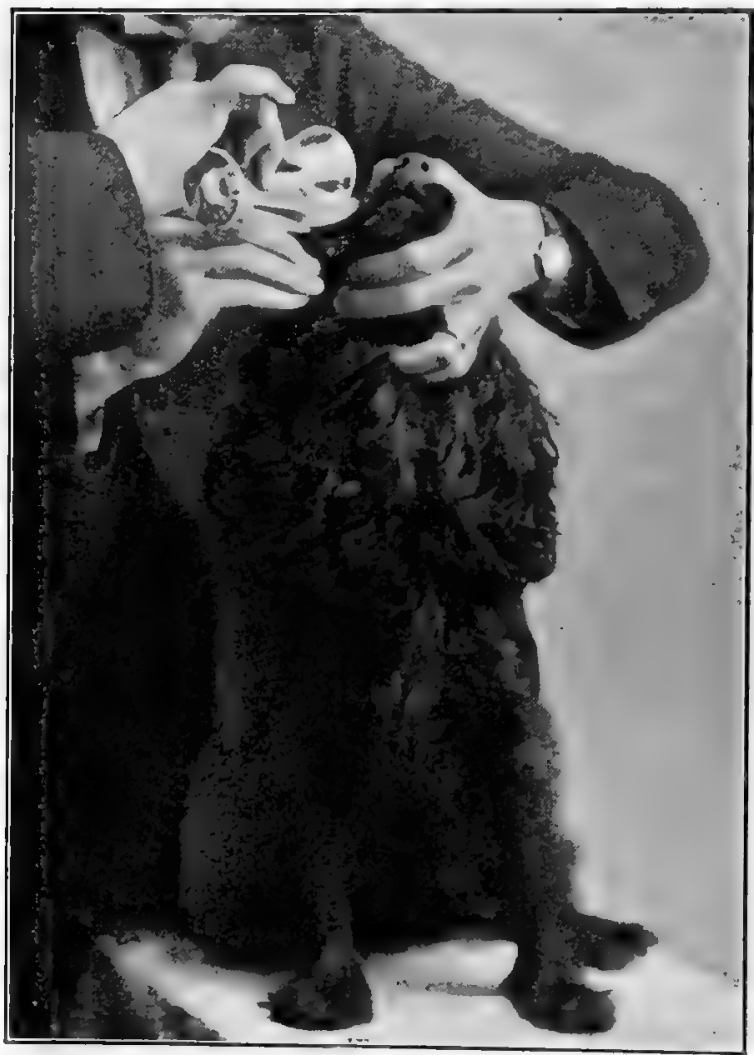

Fig. 23. -Method of giving Fluid Mridixe or Nourishaient. Nole.-The third and fourth fingers being through the collar, prevent the dog sinking down to the table.

When the catarrh in cases of distemper has extended. as far as the bronchial tubes, the practitioner must care- 
fully watch for signs of pneumonia, for if these be overlooked at the onset there is naturally an increased risk of a fatal termination.

Pneumonia.-Under the most favourable circumstances this is a very serious complication, and we cannot afford to lose any time in dealing with it.

Pneumonia sometimes appears insidiously without any premonitory cough, and on this account other gross symptoms may be allowed-if one is unwary-to distract one's attention from it. Stimulants and expectorants are given for pneumonia, and prescriptions Nos. 6 and 7 would be especially beneficial. The temperature may be high or moderately low, and additions or omissions must be made in the medicine accordingly.

Local treatment is advisable in these cases, and one or other of the following may be selected:

(a) Hot compresses to the chest repeated every fifteen to twenty minutes if necessary.

(b) Mustard applied for ten minutes or a quarter of an hour, especially in catarrhal pneumonia, and renewed at long intervals where indicated.

(c) Rubbing the chest with embrocation, camphorated oil, turpentine or soap liniment may be preferred, or even dry friction applied by hand.

Turpentine liniment consists of: Ol. tereb., I3; sapo mollis, $\mathrm{I} \frac{1}{2}$; camphoræ, 1 ; aquæ, 5 .

Counter-irritants relieve pain and internal inflammation by their irritating and stimulating effect upon the skin; and encourage absorption of exudate; their action is varied according to their strength, the duration of the rubbing, and the force used. It seems not generally recognised that the value of counter-irritants in most cases depends upon the method of their application, and efforts which are half-hearted or feeble only meet with corresponding results. 
(d) Tincture of iodine may be painted on twice daily and the chest enveloped in cotton-wool. In dealing with long-haired dogs, or in fact with any dog, it becomes really essential to clip the hair from the areas which overlie the diseased lung, for in no other way can counterirritants be adequately brought into contact with the skin. Denuding the skin of hair in these cases does not in the least expose the dog to the risk of further chill, as it is compensated for by the wool or flannel coat worn. In no case should the latter be discarded until very late in convalescence.

(e) Thermogene wool is an excellent application to the chest walls in lung complaints, and may be left in situ for a week or more without renewal. If it becomes damp for any reason, its action is intensified.

(f) Antiphlogistine paste is a remedy largely used in human practice with marked beneficial results, but for its use to be effective in dogs the hair must be clipped quite short-as is also the case with Thermogene wool. The paste is spread thickly on a piece of gauze or linen, after having been rendered semi-fluid by heat, the whole being then brought closely into contact with the chest walls while still hot, and tied in position. It should be renewed every twenty-four hours, and although expensive its therapeutic effects are undoubted.

(g) Priessnitz's compress, of German origin, has special advantages in the treatment of pulmonary disorders in dogs, since it is an efficacious means of retaining a continual dry or moist heat in contact with localised parts of the body. The desired object is achieved by first placing a layer of dry cotton-wool next to the chest (or, if moist heat is required, this wool is wrung out in hot water) over which is placed a layer of gauze to keep it in position; this is in turn enveloped in oiled silk or jaconet to retain the heat, or moisture; and finally, a bandage 
or covering of dry flannel, which prevents loss of heat by radiation.

Inhalations must not be forgotten, as they assist in the evacuation of catarrhal exudate, relieve dyspnœa, and to some extent rectify foul breath.

Narcotics are employed where the cough is very distressing, and any of the prescriptions from 2 to 5 inclusive would afford relief; some amount of cough, however, is highly desirable, as its function is to rid the bronchioles of the excessive exudations collected therein. Where the cough has subsided or become very weak, and little or no expectoration is taking place, the hypodermic injection of apomorphine (gr. $\frac{1}{20}$ to gr. $\frac{1}{5}$ ) has been recommended, though in adopting this course I must warn practitioners that as in such cases great dyspnoa and buccal breathing have probably developed, accompanied by much weakness, it is very easy to kill the patient by producing violent emesis, too large a dose causing respiratory arrest and death. The B.P. injectio apomorphinæ hypodermica is the most convenient preparation for use, as I I minims contain I grain of apomorphine hydrochloride, therefore the dose of the solution would be $m$ vss. to $m$ xxii.

Very great value has accrued from the hypodermic injection of nuclein, as it has been observed to effect a remarkable improvement in an animal's disease-resisting power, and hence it is of especial benefit in the treatment of pneumonia or pleurisy. It is prepared from yeast, and possesses the property of greatly increasing the number of leucocytes in the blood, and as they play so important a rôle in the defence of the system it is deduced that nuclein is indicated in all specific diseases of bacterial origin. Whenever fever is present, and the blood shows a low content of white corpuscles, this drug should be given. 
The dose of the 5 per cent. solution is calculated at mi. per I lb. body weight, injected subcutaneously twice daily for several days, diluted with an equal amount of physiological salt solution. A valuable auxiliary to the nuclein treatment is the injection of anti-distemper serum. (See chapter on Sero-Therapy.)

Gangrene, and Edema of Lungs.-Should gangrene or œdema of the lungs supervene, the case must be regarded as practically hopeless, and little can be done except perhaps the subcutaneous injection of ether and camphor, and application of vesicants to the sides. Finally, the animal's strength should be kept up by suitable and nourishing diet such as small quantities of scraped raw meat, or meat extract, the pure gravy from joints of roast beef, milk or peptonised foods frequently administered.

Alimentary Disorders-Dirty Mouth.-As already stated, the mouth should be regularly washed out with the remedies enumerated on p. $16_{3}$, should any hyperæmia of the gums or buccal mucous membrane be noticed or the breath become foul; and the teeth, in consequence of the dirty brown state they often assume, should also be examined and cleaned. An unhealthy mouth invariably means an unhealthy body, and treatment of distemper would not be complete or thorough without attention being paid to this important cavity.

Pharyngitis.-Pharyngitis, sometimes appearing as an extension of stomatitis or laryngitis, may be treated with mouth-washes-such as potass. permanganate, I per cent. solution; potass. chlor., 3 per cent. solution; boric acid, 5 per cent. solution; lime-water-applied either with soft swabs or through an atomiser. Warm, liquid foods and smart external counter-irritants are also indicated.

Gastritis.-In the treatment of gastritis, allusion has been already made on p. I68 to some of the drugs which prevent or control vomiting, and since this is the most 
prominent feature of gastric catarrh, it is very important. Other remedies, however, comprise the swallowing of ice, application of heat or irritants over the epigastrium, withholding of food, and the administration of one or more of the following: Opium, sodium bicarb., magnesium carbonate, hydrochloric acid, tincture of gentian, tinct. of nux vomica, bismuth subnit.

Many veterinary surgeons commence by giving apomorphine as an emetic, this being superior to and less depressing than other emetics. Where there is already great depression and emesis, the giving of calomel may be substituted, after the purgative action of which the stomach should be completely rested for a day. Then tinct. of opium can be given in doses of $m v$. to $m \mathbf{x x}$, particularly if enteritis and diarrhœa are co-existent.

Sometimes drugs in powder form are preferred, in which case a quite useful prescription would consist of :

PRESCRIPTION No. 8.

$\begin{array}{ccccc}\text { R. } \begin{array}{c}\text { Bismuth. carb. ... } \\ \text { Soda bicarb. ... }\end{array} & \ldots & \ldots & \ldots & \text { grs. v. } \\ \text { Pulv. cinnamomi } & \ldots & \ldots & \ldots & \text { grs. v. } \\ & \ldots & \ldots & \text { grs. ii. }\end{array}$

M. ft. pulv. i.

Sig. : To be dusted on the tongue and repeated three or four times daily.

The mist.bismuth. aromat. c. pepsin. (Hewlett) is also very valuable; whilst the following elixir has much to commend it by virtue of its nerve sedative action:

PRESCRIPTION No. 9.

\begin{tabular}{|c|c|c|c|}
\hline Be Pot. brom. & $\ldots$ & $\therefore$ & ... grs. $1 x x x$. \\
\hline Syr. chloral & $\ldots$ & $\therefore$ & ... Ziv. \\
\hline Tinct. cannabis indicæ & $\ldots$ & $\ldots$ & ... Mlxiv. \\
\hline Tinct. hyoscyamus & $\ldots$ & $\ldots$ & ... 3iiss. \\
\hline Tinct. gentian. co. & $\ldots$ & $\ldots$ & ... 3iv. \\
\hline Aqua ... & $\ldots$ & $\ldots$ & $\ldots$ ad $\tilde{5}$ ii. \\
\hline
\end{tabular}

Sig. : Two teaspoonfuls thrice daily. 
Probably best of all remedies, however, is chloretone in doses varying between 3 and 15 grains; cats, however, owing to a peculiar idiosyncrasy, cannot take it.

A teaspoonful of pepsin may advantageously be ad. ministered three times daily, or with the food, as an aid to thorough digestion; or tablets may be purchased containing taka-diastase (gr. i.), pepsin (gr. i.), pancreatin (gr.ss.), which will be found very beneficial in the gastric complications of distemper.

Another well-recommended preparation is that in which each fluid drachm contains :

PRESCRIPTION No. IO.

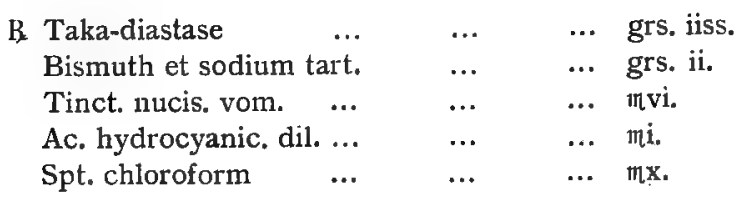

and the dose would be half to two fluid drachms according to the size of the dog.

In severe gastritis it may be necessary to resort to rectal feeding if all food taken by the mouth is immediately ejected.

Enteritis.-Enteritis will be treated more or less on the same lines as gastritis, even as regards food, warmth, and drugs. However, our efforts here are principally directed to the curtailment of its accompanying diarrhœa and tenesmus, which, if unchecked, rapidly reduce the animal to a skeleton and rob it of the last vestige of bodily strength. Diarrhœa may exist before the graver conditions of enteritis, ulceration, and intense straining, and should be taken in hand immediately, in order that these complications may be prevented as far as possible. Our aims are to reduce intestinal catarrh and consequent irritability of the mucous membrane, and to lessen peristalsis and secretion by curtailment of exercise; also 
to forbid meat and other laxative or indigestible foods, and to maintain warmth to the body.

Efficacious mixtures for the control of diarrhœa are :

PRESCRIPTION No. II.

\begin{tabular}{|c|c|c|c|c|}
\hline B. Chlorodyni & $\cdots$ & $\cdots$ & $\cdots$ & $\ldots \cdot m l x x x$. \\
\hline Bism. subnit. & $\ldots$ & $\ldots$ & $\ldots$ & ... grs. $x l$. \\
\hline Tinct. opii & $\ldots$ & $\ldots$ & $\cdots$ & $\ldots$ mlxiv. \\
\hline Gum acaciæ & $\ldots$ & $\ldots$ & $\ldots$ & $\ldots \quad z i$. \\
\hline Aquæ ... & $\ldots$ & $\ldots$ & $\ldots$ & $\ldots$ ad క̆ii. \\
\hline
\end{tabular}

Sig.: Two teaspoonfuls three times daily.

This allays gastro-intestinal irritation and is indicated in cases where the discharges are thin and copious. Dover's powder may be given for the same purpose in doses of 5 to 15 grains.

\section{PRESCRIPTION No, I2.}

\begin{tabular}{|c|c|c|c|}
\hline R Tinct. opii & $\ldots$ & $\ldots$ & ... mlxiv. \\
\hline Creosoti & $\ldots$ & $\ldots$ & ... mxvi. \\
\hline Pulv. catechu & $\ldots$ & $\ldots$ & $\ldots$ grs. 1 xxx. \\
\hline Pulv. cretæ præp. & ... & $\ldots$ & ... 3iiss. \\
\hline Aquæ & ... & $\ldots$ & ... ad 万̧ii. \\
\hline
\end{tabular}

Sig. : Two teaspoonfuls three times daily.

The above prescription is useful for chronic discharges containing mucus and blood.

\section{PRESCRIPTION No. I3.}

$\begin{array}{lccccl}\text { R Pulv. opii } & \ldots & \ldots & \ldots & \ldots & \text { gr. i. } \\ \text { Pulv. camphoræ } & \ldots & \ldots & \ldots & \text { grs. ii. } \\ \text { Pulv. ipecac. } & \ldots & \ldots & \ldots & \ldots & \text { gr. ss. } \\ \text { Pulv. plumbi acet. } & \ldots & \ldots & \ldots & \text { gr. i. } \\ \text { Excipient } \quad \ldots & \ldots & \ldots & \ldots & \text { q.s. } \\ & & \text { M. ft. pil. i. } & & & \end{array}$

Sig. : Give one three or four times daily.

Certain drugs exert an antiseptic effect on the intestinal tract-such as bismuth subgallate and subnitrate, resorcin 
(gr. i. to grs. v.), salol (grs. ii. to grs. x.), and salicylic acid (grs. iii. to grs. $x$.) -and should be prescribed in cases where the fæcal evacuations are foul. Salol is not so irritant as salicylic acid; it is not soluble in water, though freely in ether or chloroform, in either of which vehicles it may be given.

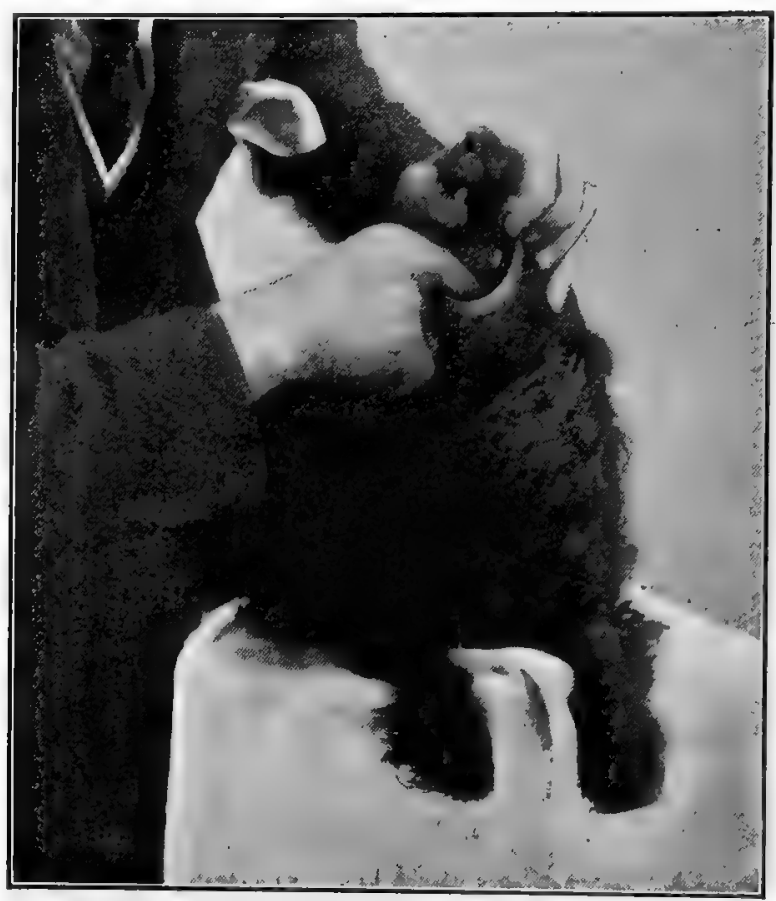

Fig. 24:-Method of Giving a Pill.

If ulceration or hamorrhage of the bowel is suspected, from the presence of blood in the stools, the administration of silver nitrate in doses of $\mathrm{gr}$. $\frac{1}{8}$ to $\mathrm{gr}$. $\frac{1}{2}$ will be followed by improvement, since this salt exerts a wonderful astringent and antiseptic effect; it must, however, be used with great care, and should be given, combined with kaolin, in a capsule. It may be followed 
up with one-minim doses of creosote, or salicylic acid (grs. ii.).

Dysentery is usually accompanied by intense straining, which, if unallayed, may lead to prolapse of the rectum. To overcome this tenesmus narcotics are indicated, such as opium, morphine, or chloretone, the latter probably proving as efficacious as any; or chloretone compound ointment (Parke, Davis) may be employed containing: Chloretone, grs. xii. ; calomel, grs. xii. ; hydrastine, gr. i.; hamamelin, grs. vii. ; lanolin base, 3i. ; supplied in collapsible tubes $w^{\text {th }}$ elongated nozzles, suitable for rectal medication.

Other agents which have been successfully tried in the treatment of diarrhœa, and from which choice may be made, are : Tannoform, grs. iii. to grs. x. ; tannigen, grs. iii. to grs. viii.; tannic acid, grs. ii. to grs. vi.; gallic acid, grs. ii. to grs. v. ; cupri. sulph., gr. $\frac{1}{2}$ to grs. ii.; ferri. sulph., grs. iii. to grs. x. ; alum. chlor., grs. v. to grs. x. ; sod. hyposulph., grs. iii. to grs. xv. ; arsenic, gr. $\frac{1}{20}$ to gr. $\frac{1}{10}$.

Astringent enemata often prove of great utility, especially in those instances in which the appetite is in abeyance, for then we may add food substances such as egg, starch, cornflour, arrowroot, etc. The medicinal enema should contain: Liq. opii. sed., mx. ; mucil. amyl., 3vi.; or lead acetate, opium, and starch gruel, at $100^{\circ} \mathrm{F}$.; in other cases the mucilage may be omitted, and the rectum washed out with solutions of tannin or alum (I to 2 per cent.), or of argentum nit. ( $\frac{1}{2}$ to I per cent.). The usual care and slowness of administration must be observed in this procedure as was recommended in the giving of nutrient clysters, and where these injections become responsible for increased irritation and tenesmus, they must be discontinued.

The food must usually be lessened in amount, given at regular intervals, of an easily assimilable and muci- 
laginous character, and consist of wheat flour gruel, cornflour and milk, white of egg, boiled milk, soup (minus fat), and perhaps meat extract, such as Brand's essence. Egg albumin is particularly good in such cases, being demulcent and soothing, as well as nourishing. Gum, starch, and white of egg are all useful mucilaginous vehicles in which to administer the various astringent or sedative drugs prescribed for diarrhœa and dysentery. As a rule the dog's water supply should be restricted.

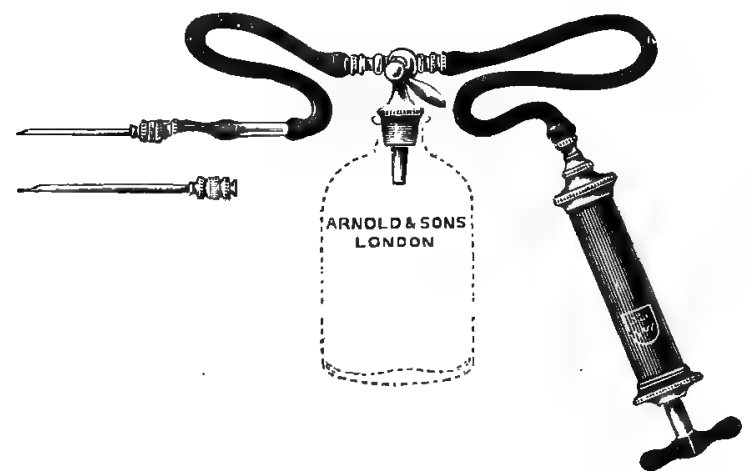

FiG. 25.-Aspirator with Escape TUbe in Receiver for USE in Paracentesis Abdominis.

"Peritonitis.-This complication is frequently fatal, but nevertheless. we must adopt some remedial measures, and the first one is the vigorous application of counterirritants to the abdomen, such as camphorated oil or mustard oil. If costiveness is present, give warm soapy enemas; then an explorative puncture of the abdomen may be made to definitely ascertain the nature of the exudate. If serous, a certain quantity should be drawn off to relieve the pressure on other organs, but if found purulent, laparotomy and thorough cleansing of the abdominal cavity must be performed, though such an operation under these circumstances will rarely prove successful. 
- Constipation.-Occasionally we have to contend with costiveness in cases of distemper, especially at the beginning, and such a condition frequently demands medicinal or mechanical interference before it can be relieved. It is often accompanied by some amount of pyrexia, and when neglected, not uncommonly leads to troublesome diarrhœa and enteritis.

Laxatives such as ol. ricini (引ss. to ji.ss.)-made less nauseous by the addition of 10 to 20 drops of ætherhydrarg. c. creta (grs. iii. to $x$.), or cascara sagrada ( $m v$. to $m(x$.$) , will usually have the desired effect, but where$ they fail, it is unwise to administer further purgatives without allowing two or three days to elapse. Better resort to the use of mechanical lubricants, such as liquid paraffin and glycerine, or glycyrrhiza, the former being especially favoured in some quarters. It has no action whatever on the bowel other than that of mechanical lubrication and protection, for it undergoes no alteration during its passage through the alimentary canal.

In cases of distemper it is best prescribed in the form of either petroleum emulsion (containing liquid paraffin and the hypophosphites of calcium and sodium), dose I to 4 fluid drachms, or as the proprietary "Virolax" (60 per cent. paraffin and 40 per cent. Virol), which is not only a laxative and intestinal lubricant, but also a valuable food of pleasant flavour.

Many veterinarians prefer the employment of enemas of warm water in which a little soap has been dissolved, or rectal injections of liquid paraffin, plain olive oil, or a solution of glycerine and water ( $\mathrm{I}$ to 10 ). Suppositories of glycerine are very convenient in cases of obstinate constipation, and since a good many instances of this condition arise from the torpidity or dry condition of the terminal bowel, the medicant reaches the seat of the trouble with good reșults. 
Much may be done, however, for the relief of costiveness by the judicious selection of foods, and frequently no resort need be had to drugs or other agents whatever. The continued use of moist foods, such as biscuits soaked in warm water or soup, will often prove all that is necessary; or the principal meal may consist of biscuit moistened with soup, and a proportion of green vegetable and meat added. Meat and liver are laxative, and if mixed with brown bread, greens, and broth, they usually form an efficient aperient of themselves, especially if any exercise can simultaneously be taken, which is not, however, the case in distemper.

A teaspoonful to a tablespoonful of salad oil given alone or added to the food once daily for several days is harmless and often quite useful.

Many dog owners labour under the mistaken impression that a lump of sulphur placed in the animal's drinking water regulates its bowels and "purifies its blood." Sulphur is insoluble in cold water, thus to exert any laxative effect it must be taken internally either in pill or powder form.

Jaundice.-If jaundice appears during the course of the malady we must aim at eliminating its cause-viz., duodenal catarrh-and the relief of any accompanying constipation by the administration of saline laxatives and warm enemas. Calomel in small but frequent doses $\left(\frac{1}{2}\right.$ to $\frac{3}{4}$ grain) has been well recommended on account of its emetic property and of its intestinal disinfectant action. Muiller and Glass advocate the mechanical emptying of the gall-bladder by pressure of the abdomen between the fingers, or by faradisation-i.e., the therapeutic use of induced currents applied in the region of the liver on both sides of the abdomen for ten minutes at a time twice daily. Other helpful remedies are massage or the application of counter-irritants, such as linseed poultices 
or mustard, to the abdomen. If the patient is wealk give nitro-hydrochloric acid ( $m$ iii. to $m x$.) with quinine (grs. i. to grs. v.), which is soluble in acid, or a combination of camphor, ether, and caffeine.

The use of a bacterin prepared from the cultures of $B$. coli communis has been reported of considerable value in the treatment of catarrhal jaundice, but I have not personally exploited it, nor have I' any detailed or confirmatory information before me. It appears quite feasible, however, that such a bacterin might have a marked beneficial effect.

Nervous Disorders. - In probably the majority of cases in which these grave complications malse their appearance, complete recovery is not effected. Much depends upon the type of disorder, the stage at which its onset is first noticed, and its severity.

Convulsions.-Convulsions, when they appear at the commencement of the disease, are doubtless not so serious and may usually be checked by the use of narcotics, and I know of no better agent for the purpose than chloretone, which may be given in large doses at first, then gradually reducing the amount as the symptoms are allayed. A light, easily digestible diet, perfect quiet and semi-darkness, and free action to the bowels, are auxiliaries in the treatment of fits. Chloroform inhalations, the bromides of potassium, sodium, and strontium, sulphonal (grs. x. to grs. 1.), chloral hydrate, phenazone (grs. $\mathrm{x}$. to grs. $\mathrm{xx}$.), morphine subcutaneously (gr. $\frac{1}{3}$ to gr. $\left(\frac{1}{2}\right)$, ice to the head or spine, are remedies which have been variously recommended for convulsions; all these, with the exception of chloroform, are, in my experience, inferior to chloretone. Sodium bromide (grs. vii. to grs. xx.) certainly appears to lessen the severity and frequency of each convulsion, and can be given in full doses over a long period of time. 
If the fits follow one another very rapidly, thereby becoming very exhausting, chloroform may be administered to obtain an immediate effect. Provision must be made to prevent the dog from injuring itself. A convenient and ready-made nerve sedative is the Bromide Compound Elixir (Parke, Davis), containing pot. brom., chloral, ext. cannabis indica, and ext. hyoscyamus, the Indian hemp of which is standardised by physiological test. If chloral hydrate is given alone, it must be either very well diluted or suspended in mucilage, owing to its excoriating effect on mucous membranes.

When the convulsive fits appear for the first time very late in the disease, the matter is much more ominous, and we usually see chorea or paralysis as a sequel.

Chorea.-Subjects of severe chorea seldom regain their normal health, but in moderate cases I have known quite a number to absolutely recover, even though the process may have extended over quite a considerable time, from one or two months to as many years.

Whether the results can have been ascribed to the treatment applied or to Mother Nature, is difficult to tell, but my remedies have been restricted to the administration of nerve and other tonics-such as Easton's Syrup, triple syrup, glycerophosphates with iron, arsenic, quinine, and phosphorus, etc.-combined with fresh air, hygienic surroundings, liberal and nutritious meat diet, with a fair proportion of milk or other fatty matter. These nerve stimulants, however, must not be employed until the animal has thoroughly lost all other traces of distemper and regained its former strength to a large extent. Then a course of Easton's Syrup may be tried, starting with small doses and slowly increasing them to the maximum permitted for the particular breed of dog. For instance, a King Charles spaniel would take as a 
commencing dose about 6 minims thrice daily; a Pekinese would take 9 minims; a fox terrier, I 2 ; an Airedale, I 5 ; a collie, I 8 ; and a St. Bernard, 20.

After the first week the dose in each case may be slightly increased, and the medicine should be continued for at least a month. Since Easton's Syrup contains strychnine (gr. $\frac{1}{32}$ to each fluid drachm), which is a stimulant to the motor centres of the spinal cord, it will be readily appreciated how carefully the dose must be regulated, for full doses will produce tetanic convulsions, whilst small or medicinal doses are nerve tonics and antiparalysants, and their use is not indicated until the severe choreic spasms have abated somewhat.

Arsenic has proved of considerable value in the treatment of chorea and epilepsy, for which in human beings it has been regarded as especially serviceable. According to Finlay Dunn, its action in these cases appears to depend partly on its alterative properties and partly on its diminishing irritability of motor nerves. It should be given in full doses, well diluted, three times a day after meals, but the dose should be graduated from a small dose at the beginning to a full medicinal dose at a later period, for it is only in this way that an animal will be enabled to develop a tolerance of doses which, if administered at first, might prove fatal.

Dogs will take gr. $\frac{1}{30}$ to gr. $\frac{1}{10}$, according to their size, or Fowler's Solution (which contains I grain in I IO minims) may be given in doses of miii. to $m$ xii.

Sometimes it is desirable to cease its administration after about a fortnight, especially when indigestion or diarrhœa becomes manifest, owing to its cumulative tendency; it may then be recommenced after seven or eight days, and during the interval some preparation of iron should be prescribed, particularly ferri redac., which is less liable to cause digestive derangements than 
are the other iron salts. Fowler's Solution may be conveniently added to the dog's saucer of milk, being practically tasteless; the reduced iron can be similarly mixed with food, as it is quite tasteless and nonastringent.

Quinine is a most useful tonic in debility or nervous weakness, though it does not affect motor. nerves unless actually brought in contact with them. It is especially indicated for weakly, ill-nourished dogs, when given in conjunction with iron salts and cod-liver oil. Doses of quinine (grs. v. to grs. $\mathrm{xx}$.) may be given two or three times a day for several days, or smaller doses may be continued over a more prolonged period. Small doses have usually a more potent tonicity than large ones.

Some writers have recommended counter-irritation over the affected muscles, but since the spasms of these muscles are not of local origin, little benefit can be expected of such a procedure; better far to stimulate the seat of the trouble by the application of irritants to the spine, if the principle of counter-irritation is to be employed at all. This can be accomplished by clipping the hair from over the entire length of the spinal column, and vigorously rubbing in cantharides ointment, biniodide of mercury ointment, or strong ammonia and turpentine liniment.

I do not approve nor practise the insertion of setons in such cases, as I consider they are far too drastic and of very doubtful value.

Electricity has frequently proved its efficacy when regularly applied along the vertebral column, and is a therapeutic measure which should be resorted to in all cases where the patient is of great pecuniary or sentimental value.

As regards the treatment of chorea in its acute or early stage, we must aim at the speedy reduction of nervous 
irritability, in default of which the patient may succumb from sheer exhaustion. Potassium bromide, chloral hydrate, and morphine have little effect on the spasms, but cannabis indica or chloretone internally, and chloroform or ether by inhalation, have a decidedly greater influence, and may be usefully employed in urgent cases.

"French veterinary surgeons have prescribed antipyrin (or phenazone) as a carminative in irritable conditions of the central nervous system, and as a general analgesic as well as a local anæsthetic in neuralgic cases" (Finlay Dunn). .It has been extensively used against chorea in man with much benefit, but, unfortunately, seems to be attended with less favourable results when applied in canine practice.

Other well-tried, though probably less efficacious, remedies are: aspirin, pot. iodide, ammonium and sodium bromide, sodæ. salicylate, argentum nitrate, belladonna, hyoscyamus, antifebrin, camphor, and codeine. If the animal is of little value, and severe choreic spasms develop, it would doubtless be kinder and more economical to advise destruction than to administer a very prolonged treatment in the hope of ultimate recovery.

On the other hand, such recoveries do occur, even after bad attacks, and if the owner expresses anxiety that every endeavour be made to save the dog, the practitioner cannot do better than follow the course of treatment herein described.

Paresis._In paresis, however, whether it be hemiplegia, paraplegia, or general paralysis, the employment of nerve sedatives is contra-indicated, and we rely instead upon stimulants and tonics, usually administered patiently over a long period.

Dogs frequently wholly recover the use of their muscles 
or limbs when accorded adequate and painstaking treatment. Easily digested nourishing food must be given, and the bowels kept well open, whilst once at least every day the dog should be assisted to stand or walk for a short time in order to bring the affected muscles into play, and help to restore their normal functions. Mild stimulation of the spinal column by counter-irritants may be occasionally tried, and once daily electricity should be applied to the affected parts after having first damped them with salt solution. For instance, if a limb is paralysed, one pole of the battery should be placed on the middle of the back and the other on the foot, allowing a current of gradually increasing strength to pass through for about ten minutes. Massage is useful, as also are warm baths, and where there is a tendency to retention of fæces and urine we must evacuate same by means of enemas and the passage of the catheter respectively. If there is paralysis of the sphincter muscles, urine and fæces will be involuntarily voided; but as the body recovers its nervous tone under the influence of time and suitable drugs, etc., this incontinence will, as a rule, disappear.

Since large amounts of fluid exudate have from time to time been found on the cord at autopsy, it is recommended that pot. iodide should be internally administered, in doses of grs. ii. to grs. $x$. twice daily, as a means of promoting reabsorption. The same effect results from the subcutaneous injection of eserine combined with pilocarpine.

As a tonic, strychnine should be prescribed with arsenic or quinine, or either used alone. Strychnine may be given in the form of Easton's Syrup or as tincture of nux vomica ( $m v$. to $m$ xii.), or one daily hypodermic injection of liquor strychninæ ( $I$ to I Io) in doses of $m$ i. to $m v$. This alkaloid is very cumulative and toxic; thus it is 
essential to omit the injections for a couple of days after they have continued for five or six days.

It is conceivable, though doubtful, that the loss of hearing, scent, and voice, sometimes accompanying general paralysis, would be regained upon recovery from the primary paralytic condition. The amaurosis so arising at least appears to be always permanent and progressive, no treatment having any very decided effect. Eserine drops, however, may be tried, but with doubtful benefit.

Cutaneous Lesions.-As a rule no treatment is required for the skin lesions of distemper, since they are mostly quite benign and transient. Nevertheless, as in rare instances we find the vesicles extending and coalescing, resulting in the formation of comparatively large moist sore areas, we may either bathe the places with a warm solution of Sanitas or Condy's Fluid, or may resort to antiseptic dusting powders such as mixtures of zinc oleate 2, kaolin 3, and boracic acid 5 ; or Fuller's Earth 5 and boracic acid 5 ; or zinc oxide I with talc powder Io; dusted on twice daily with cotton-wool.

Defective Heart.-The deficient valvular action occasionally observed as a result of distemper or other infectious diseases, and particularly of chronic nephritis, must be considered as incurable, although we may endeavour to aid the organ by restriction of exertion and excitement, and, where the heart is greatly weakened and respiration more difficult, by administration of heart tonics such as tincture of digitalis ( $m v$. to $m \times x$.), tinct. strophanthus ( $m$ ii. to $m x$.), caffeine citras (grs. ii. to grs. viii.), three times daily. The resulting dropsy must be relieved by giving diuretics such as liq. ammon. acet., spts. æth. nit., pot. nit., or pilocarpine hypodermically.

A useful mixture for this complaint would thus consist of : 


\section{PRESCRIPTION No. I4.}
B. Tinct. digitalis
$\begin{array}{llll}\ldots & \ldots & \ldots & \text { mlxxx. } \\ \ldots & \ldots & \ldots & \text { ziii. } \\ \ldots & \ldots & \ldots & \text { grs. xxiv. } \\ \ldots & \ldots & \ldots & \text { ad }{ }^{\mathrm{i}} .\end{array}$
Spt. æth. nit.
Caffeine cit...
Aquæ

M. ft. mist.

Sig. : One teaspoonful three times daily;

or-

PRESCRIPTION No. 15 .

\begin{tabular}{|c|c|c|c|}
\hline $\mathrm{R}$ Tinct. digitalis & $\cdots$ & & ... mlxx. \\
\hline Tr. strophanthi & $\cdots$ & $\cdots$ & ... mxxxii. \\
\hline Tr. nuc. vom. & $\ldots$ & $\ldots$ & ... mxl. \\
\hline P. pot. nit. ... & $\ldots$ & $\ldots$ & $\ldots \quad 3 i_{.}$ \\
\hline Aquæe & $\ldots$ & $\cdots$ & $\ldots$ ad 3 i. \\
\hline
\end{tabular}

Sig. : One teaspoonful three times daily.

A liberal diet must be allowed and constipation always guarded against.

Nephritis.-Nephritis as occurring in dogs is not so significant as in man, and frequently it exists without ever being diagnosed. The little one can do for it is confined mostly to ridding the body of its moisture through other channels, so as to rest the kidneys as much as possible.

To accomplish this, cascara or calomel may be given as purgatives, or excretion by the skin stimulated by the application of warm moist clothing, Priessnitz bandage, or by hot baths, etc. Spices, salt, and diuretics must be avoided, and if there is great pain and arching of the back, hot linseed poultices may be applied in the region of the kidneys. Barley-water should be substituted for the ordinary drinking water, and urotropine given three times a day as a urinary antiseptic, in doses ranging between grs. v. and grs. xv.

Cystitis.-Inflammation of the bladder may be treated locally as well as medicinally; in fact, if the urine con- 
tains many pus cells or bacteria, the local treatment becomes essential. It consists in irrigating the bladder with weak antiseptic solutions such as potass.'permanganate I per cent., boric acid 2 per cent, or zinc sulphate I per cent., by passing a sterile catheter into that organ, attached to which is a small rubber pipe and

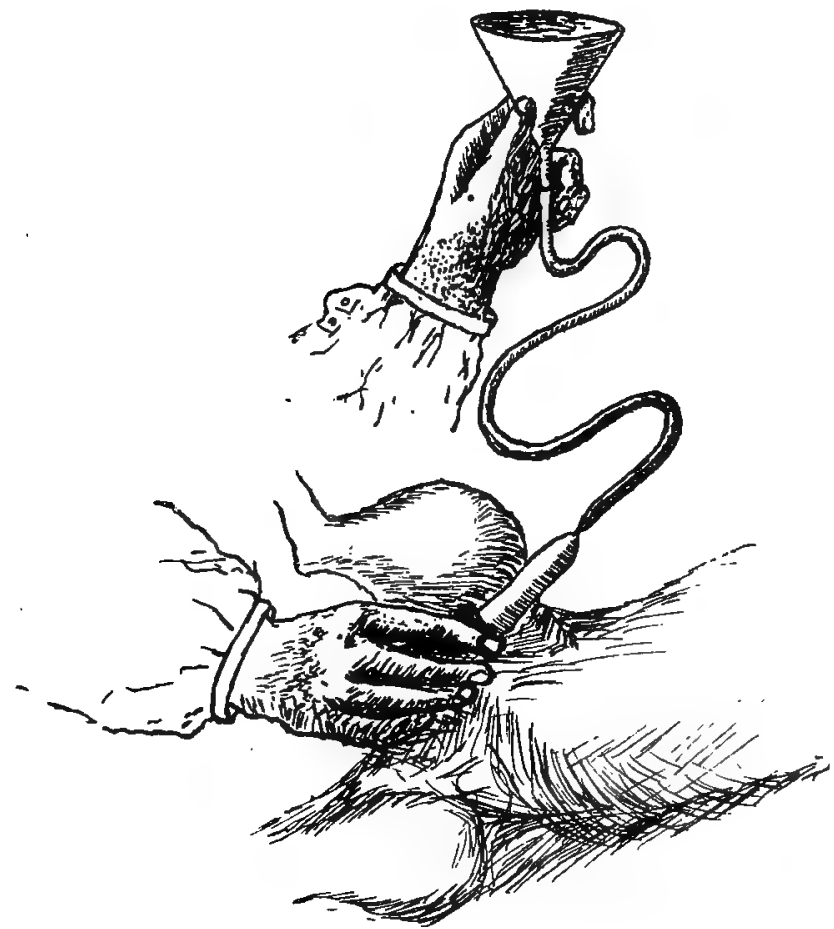

Fig. 26.-IRRigating the Bladder.

The procedure is the same as for rectal lavage.

funnel (see Fig. 26), the latter being filled with the warm solution, which then gravitates slowly into the bladder; by lowering and inverting the funnel the liquid runs out again exactly in the same way as it does in the performance of rectal lavage.

If preferred a syringe may be attached to the catheter, 
and the liquids forced in and drawn out by its aid. (N.B.-Of course, the bladder is emptied before any solution is introduced into it.)

If there is much hæmaturia a 2 per cent. solution of tannic acid may be injected. Internally one should administer salol (grs. ii. to grs. x.), salicylic acid (grs. iii. to grs. x.), urotropine (grs. v. to grs. xv.), pot. nitrate (grs. vi. to grs. xx.), benzoic acid (grs. iii. to grs. x.), copaiba ( $m x$. to $3 i$ ), or infusion of buchu (zss. to $3 i i$.) with oleaginous laxatives.

Salol and urotropine are particularly useful, and I prescribe them four or five times daily in every case of bladder implication, especially when accompanied by pain. It may be mentioned in passing that urotropine is the proprietary name for hexamethylene, and if purchased under the latter name is considerably cheaper.

Glass recommends morphine or bromide of soda in cases where there is involuntary or constant urination. If the condition is acute, hot poultices or Priessnitz compresses may be applied over the region of the bladder.

Urino-Genital Organs.-The catarrhal or purulent discharges emanating in rare instances from the vagina of bitches and penis of dogs, are usually soon checked by injections of antiseptic solutions, the best of which are corrosive sublimate (I to 4,000), zinc sulphate I per cent., and permanganate of potash I per cent.

Otitis.-Before any therapeutic agent can be used, the ear must be thoroughly cleaned out, for it will be found to contain brownish crusts and an offensive waxy or purulent discharge, which must all be removed by means of cotton-wool attached to the end of a pair of fine spring forceps. Dipping the wool in spirit, such as æther, methylated spirit, or chloroform, aids greatly in dissolving the ear discharges, and after their complete 
removal a warm lotion composed of liq. plumbi subacet. (I to 40) may be dropped into the ear twice or thrice daily, the base of which is then worked about in a circular manner in order to bring the medicant in contact with every part.

A very good and soothing application consists of Goulard's Extract, I part; olive oil, I2 parts. Where

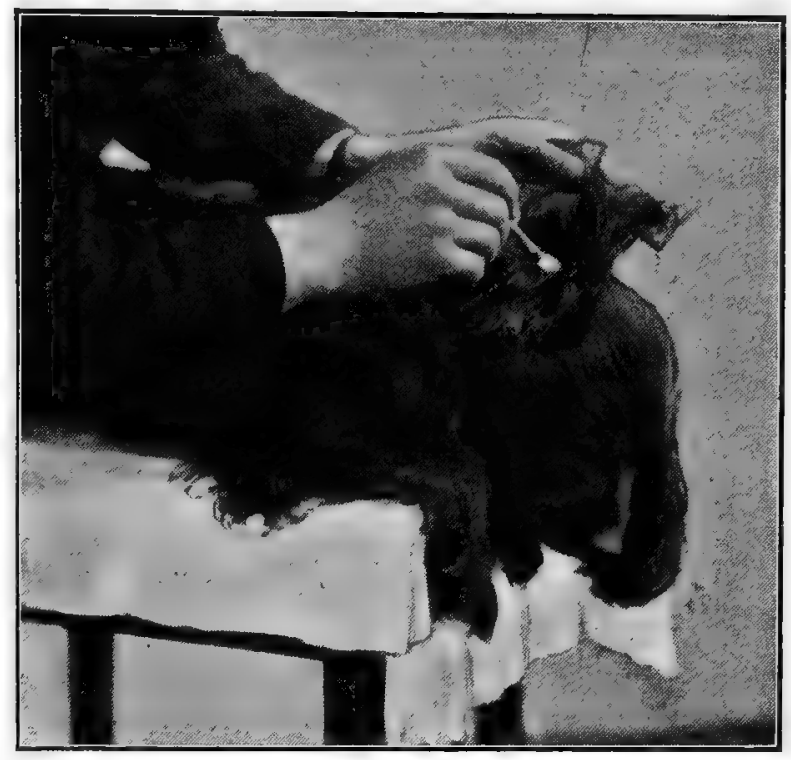

Fig. 27.-Cleaning the Interior of the Ear.

the ear is particularly sensitive, we may apply a few drops of a 4 per cent. solution of cocaine before commencing to manipulate the organ, or this may be added to the prescribed lotion. A useful astringent and antiseptic ear-dressing for cases accompanied by much ulceration and inflammation is made with I part of tannin in Io of glycerine, which must be applied several times before any benefit is noticed to accrue.

If the ulceration is deep-seated and difficult to see or 
reach, liquids will probably prove the best treatment, otherwise I prefer to employ dry applications, such as various powders or soluble "conoids." Some very efficacious powders for dusting into the ear are those consisting of-(a) Zinc oleate, I; boracic acid, 5 . (b) Ground starch, kaolin, and boracic acid in equal parts. (c) Salicylic acid, I; talc powder, 20-and these should only be introduced after the meatus has been first cleaned with one of the recommended liquids, and thoroughly dried with soft cotton-wool.

For some years I have had a special preparation made for ear cases, which has proved very valuable and easy of administration. The formula is-

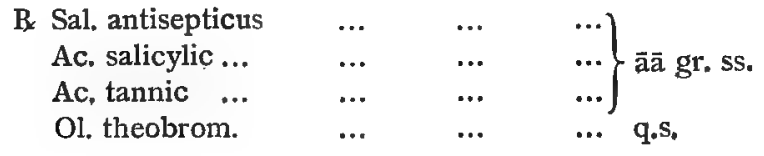

and it is put up in conical form. A cone is placed as far as possible into the ear, followed by a plug of dry cotton-wool to keep it in position. The heat of the ear rapidly dissolves the base, and the medicants are set free to exert their soothing and very beneficial effect upon the tender ulcerated surfaces:

It is inadvisable to probe about in the depths of the meatus except where it is absolutely essential, as in the preliminary cleansing process, and then it may only be attempted with the utmost care and consideration for the feelings of the dog.

Strong or irritant dressings are to be condemned for use in the auditory meatus, as granulations seem particularly liable to occur. This has been observed to follow the too frequent use of hydrogen peroxide as an ear lotion. If the irritation is intense, and the dog is seriously damaging its ear through violent shaking or 
otherwise, it may become advisable to apply a special leather cap to prevent such injury (see Fig. 28).

Suppurations.-The suppurations which may on rare occasions appear during the course of an attack of distemper are conditions which must be treated surgically, and unless they assume alarming proportions, they are usually readily amenable to treatment.

In the latter event, however, we may confer great benefit by the subcutaneous injection of nuclein daily

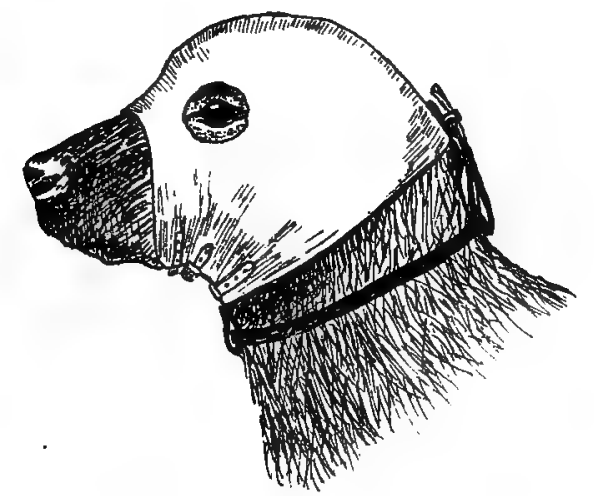

Fig. 28.-Leather Cap for Protecting the EARs.

The cap may also be made of calico.

for several days, or may resort to bacterin treatment. For this a bacterin made from cultures of Staphylococcus albus and aureus and streptococcus (all of canine origin) is used. Five or six doses of increasing strength are inoculated subcutaneously in the hairless region of the abdomen or inside the thigh, at intervals of three or four days.

\section{SERO-THERAPY}

The possibilities of active immunisation have already been discussed under "Prophylaxis," and mention of passive immunisation or antitoxic treatment has been 
reserved for the chapter on "Therapeutics"; for no work would be complete without reference to this most important method of treating disease. Antitoxic treatment is that which neutralises the toxin already circulating in the blood before it has entered into combination with the tissues. Most sera are anti-microbic, and bring about destruction of the microbes, thereby preventing them from forming toxin.

It has been claimed that considerable benefit may result in the treatment of distemper by the inoculation of immune serum, though it is recommended that this should be accompanied by inoculations of anti-distemper vaccine. It may sometimes be a matter of uncertainty as to whether a bacterial vaccine or a serum should be used in a given case, and the former may occasionally be administered when the latter would be more suitable. Sera give the best results in acute general infections, when the condition of the patient is such that it cannot produce its own antibodies, and it is necessary to supply them by the injection of antitoxin or anti-bacterial serum.

At the onset of an infectious process, however, the disturbance is generally local, and at such a time the prompt use of a suitable bacterial vaccine is indicated.

Thus the nature of an infection is the guide as to whether a serum or bacterin should be employed, the former being indicated in general infections, and the latter in localised or semi-localised infections. Too often the case is not seen until the dog is so ill that its system can scarcely respond to a vaccine and produce sufficient antibodies to combat the toxins produced by the infecting microbes. In such a case the supply of antibodies ready formed-as provided in anti-distemper serum-will be of great value.

Many sera, notably those of Piorkowski and Lignières, 
have from time to time been produced, tested, and proclaimed infallible by their promoters, yet many users have pronounced against their efficacy. In any case they can do little harm, but may on the other hand do considerable good, and I unhesitatingly recommend a trial, having first obtained what is considered the best available product.

The latest, and therefore probably least known, serum is that of Ferry, which is obtained from the blood of animals that have been immunised against several strains of $B$. bronchisepticus. This serum may be given at any stage of the disease in doses of 5 c.c. daily, or, if the case is severe, twice daily. Ferry states that in the early stages doses of serum may be alternated with doses of vaccine with very good effect.

Polyvalent antistreptococcic and antistaphylococcic sera are now obtainable, of canine origin, which should have a marked salutary effect when employed against secondary infections, for we know that in nearly every fatal case it is the complication and not the primary infection which directly brings about death, and that the causal organisms of these complications are frequently cocci.

However, since Ferry's mixed bacterin contains $B$. bronchisepticus, staphylococci, and streptococci, it follows that if an animal is immunised with this bacterin, the immune serum obtained from that animal's blood should possess antibodies which would not only neutralise the toxins of $B$. bronchisepticus, but also those of the cocci named; and this property is a distinct advance on all previous sera, which have never exerted any influence whatever on secondary infections. 


\section{CONVALESCENCE}

This matter, though last, is not least important, for it very often depends upon the care with which an animal is nursed through convalescence whether it will thoroughly regain its normal health and become robust and hardy, or whether it will succumb to a relapse, or linger on from month to month as a weakly, emaciated wreck.

As a dog shows increasing appetite and desire for solid foods, it is inadvisable to yield to its cravings too readily; but any increase in quantity, or change from liquid to solid, should be effected with the tardiest graduation, remembering always that the animal has survived a most trying ordeal and emerged with every organ in a much reduced and delicate state, The jacket should not be removed until the last symptom has disappeared, and then only for short periods at first, which may be lengthened by degrees until it is discarded altogether. Exercise too should be only grudgingly allowed in the beginning, until it eventually becomes apparent that strength has returned to the limbs, and that the dog is not exhausted after activity.

In all these matters one should be guided entirely by what one would consider advisable or good for a human being who has been long confined to bed, the subject of a very debilitating and exhausting disease.

The food should continue to be nourishing and easily digestible, and should be administered little and often for a week or two, then gradually increased to normal in quality and frequency.

No bathing should be undertaken until the dog is thoroughly strong and well. If the coat is much soiled, this had better be remedied by the use of dry shampoos applied with caution.

Permit no draughts, walks in the rain, or any other 
condition or exposure which might be likely to lead-to a chill or a relapse; and allow no other distempered dog to come in contact with it, for immunity may not yet have become firmly established. Also exercise consideration for the health of other, unaffected, dogs, since the convalescent one will be infective or a "carrier," probably for some weeks, or at any rate until the last visible symptom has departed at least a week, and the cured dog has been thoroughly immersed in a disinfectant bath. All its old rugs, coats, bedding, and other articles pertaining to the sick-room, must be burnt, baked, or otherwise disinfected.

Tonics, particularly quinine, may now be administered as recommended on p. 200 , and attention should be paid to the action of the bowels.

Nothing can be hurried in the therapeutics or aftertreatment of dogs suffering with distemper-all must take its course. 


\section{APPENDIX I \\ EXTERMINATION OF RATS}

The safest way to exterminate rats and mice is with Liverpool Virus, though I cannot pretend it is the most efficacious. Experience has taught me that rats will not readily eat this paste, but show a marked preference for phosphorus pastes spread on bread, and with the latter method I have been very successful. However, phosphorus, being very poisonous, is a dangerous agent to spread in the vicinity of dogs, and should only be used after first making sure that every dog is secure in its kennel, counting the pieces of bait laid down at night, and collecting in the morning what the rats have not touched. Water should be placed near the phosphorus, as this aids the quick action of the latter. Liverpool Virus can be safely scattered among dogs, as it has no pathogenicity for any but rodents. Another efficient method of killing rats is to mix dry oatmeal in equal proportion with plaster of Paris, and leave it on the floor with a basin of water beside it. The rats greedily eat the powder, become thirsty, drink, and then die. Needless to add, all access to dog biscuits should be curtailed, these being stored preferably in covered zinc bins, for so long as rats can feed on good wholesome biscuits, they will not choose phosphorus or any other bait. 


\section{APPENDIX II}

\section{DISINFECTION}

$A$ disinfectant is an agent which destroys pathogenic germs, or renders ferments inactive; it is distinguished from antiseptics in that the latter do not necessarily destroy but inhibit the growth and multiplication of putrefactive organisms, and from deodorants by the fact that these remove undesirable or offensive odours, without the surety of killing the microbes, or hindering their growth. Deodorising is not accomplished by merely substituting one smell for another in a room where putrefactive processes are generating a totally different odour. True deodorants attack the cause of the evil smell, although they themselves may be odourless; examples are charcoal, Condy's fluid, hydrochloric or nitric acids, chlorine, etc. Many disinfectants are also deodorants, though few deodorants could be termed disinfectants.

Disinfectants exert their bactericidal effects in various ways, viz.: By oxidation, e.g. potassium permanganate, ozone, chlorinated lime, hydrogen peroxide; by coagulation and desiccation, e.g. metallic salts such as silver nitrate, corrosive sublimate, etc.; by protoplasmic poisoning, e.g. carbolic acid and its various derivatives.

Many disinfecting agents are very toxic, and dogs are peculiarly susceptible to poisoning from certain varieties by absorption through a wound or the skin; hence careful choice must be made according to whether the disinfectant is to be used for cleansing walls, floors, benches, and other objects, or will be employed in the dog's bath water, or to sterilise wounds, etc. Carbolic acid, some tar preparations, and the various mercuric germicides, are all dangerous to the dog, and should be used with great caution. Disinfection may be carried out by a variety of means, such as fumigating, spraying, baking, boiling, chemical solutions, sunlight. 
Fumigation.-Buildings or rooms may be disinfected throughout by means of fumigation with poisonous gases, and to be effective the rooms must be entirely closed, so that no outlet remains for the premature escape of the fumes generated. All windows, fireplaces, ventilators, and doors must be sealed by stuffing paper in their cracks and crevices, after which the gases are liberated and allowed to pervade the air for about twentyfour hours. Sulphurous acid gas can be produced by burning sulphur on a red-hot iron plate; or the sulphur may be placed in an old iron saucepan, moistened with a little methylated spirit, and ignited; or sulphur candles manufactured for the purpose may be burnt. The fumes of sulphur, however, are not really very efficacious, as I have known flies and spiders on two or three occasions to recover, even after several hours' exposure. Further, the fumes have a decidedly deleterious effect on metals, and for this reason are frequently impracticable. For a small room, the burning of about $\mathrm{I} \frac{1}{2}$ lbs. is usually considered sufficient, but it has been found that if bacteria are protected-i.e., when they are in the middle of small bundles of clothes, rugs, etc.-no effect is produced on them, even by an atmosphere containing a large proportion of the sulphurous acid gas. To fumigate I,000 cubic feet, $5 \mathrm{lbs}$. of sulphur are required.

Formalin.-Probably the best fumigator is formalin, which can be obtained in tablet form, the tablets being placed in a receptacle beneath which is a methylated spirit stove. The amount of formalin required is governed by the cubic space of the room, and it is advisable to allow twelve hours to elapse before throwing open the windows and doors. Its fumes are colourless and odourless, and cause no damage to metals, furniture, or hangings. Its effect on dry organisms is very much less than on wet ones, so that a moist atmosphere would appear to be helpful. Another method of producing formalin gas is by mixing together $20 \mathrm{ozs}$. of formalin and $16 \mathrm{ozs}$. of permanganate of potash crystals. 
This amount is sufficient for the disinfection of $i, 000$ cubic feet of space.

Care should be exercised in its use, as the gas evolved is slightly inflammable. After the operation, the person entering to open sealed windows and doors should wear a wet towel over the head, as the fumes are very deadly. Chlorine gas is a fairly effectual disinfectant, and sufficient for 1,000 cubic feet of space would be liberated by carefully adding 6 ozs. of strong sulphuric acid to about 2 lbs. of chlorinated lime.

Spraying.-The dissemination into the air of a sickroom of antiseptics in a fine state of division cannot but have marked beneficial results, especially to patients suffering from respiratory complaints, and as applied to canine distemper wards it might very usefully be resorted to much more than it is. Special spraying machines may be purchased for the purpose, and some of the substances used with them are formalin, eucalyptus, Sanitas, euthymol, or almost any of the volatile oils. They not only deodorise and refresh, but largely sterilise the atmosphere of a sick-room. It is stated that I part of formalin in 10,000 of air will kill the cholera vibrio in one hour, the diphtheria bacillus in three hours, and the Staphylococcus pyogenes in six hours. Formalin being an irritant, the sprayer should wear gloves and goggles. A solution of $\frac{1}{2}$ per cent. to I per cent. is used. Perchloride of mercury is one of the substances which have been used for disinfecting rooms by distribution from a spray-producer, of which the "Equifex" may be taken as a type. With such a machine it is calculated that $I$ ounce of hydrarg. perchlor. used in solution of $I$ in r,ooo will disinfect 3,000 square feet of surface.

Baking and Boiling.--Sterilisation by heat is a process seldom resorted to by those who devote their time and energy to the care of sick dogs, as so much of the apparatus pertaining to dogs - such as collars; leather leads and muzzles, clothing, dishes, etc.-is unsuitable 
for boiling or baking, and can better be sterilised by washing or soaking in strong disinfecting solutions.

Application of Chemicals. - A large number of chemical substances have germicidal properties, but instead of classifying them according to their mode of action or otherwise, I shall serve the purpose of this book-and probably of its reader-better if I indicate, under separate sub-headings, the uses we have for these substances, and the chosen agents in each case.

(a) Disinfection of Yards, Floors, Walis, Benches, Dishes, Leather-Ware, Drains, etc.-For these external purposes we need not entertain the same scruples in our choice of a disinfectant as would be imperative did we intend it to come into direct contact with the living animal. Consequently the properties of the most toxic of germicides may be exploited, and of these doubtless perchloride of mercury (corrosive sublimate) is the most powerful and useful. A I in 100 solution will kill anthrax spores in twenty minutes, whilst the bacilli themselves in blood will be destroyed after a few minutes' exposure to a I in 2,000 solution. It is generally used in the strength of $I$ in $I, 000$, which practically instantaneourly kills all vegetative organisms. The most popularly favoured disinfectants, however, appear to be the various products of the distillation of coal, probably by virtue of their aromatic qualities no less than of their undoubted cheapness and efficacy as bactericides. Carbolic acid (phenol) in a 3 per cent. solution with water will kill all ordinary bacteria in the vegetative condition (and of these the staphylococcus is most resistant) in less than five minutes; but it is irritant and toxic, and in its pure state is too expensive for wholesale use, and, like its cheaper and crude form, is not readily miscible with water. The impure form, moreover, has an oily. character, which renders it not so convenient or pleasant for washing various articles in. water, but it may be applied undiluted with a brush over doors, walls, or floor-boards. Kaufmann states that creolin (Jeyes' Fluid) 
as a bactericide is ten times as powerful as carbolic acid; and, unlike the latter or creosote, ro per cent. solutions do not irritate the skin or the mucous membranes. Creolin is a coal-tar product saponified with resin and alkalies, and mixed with forty or more parts of water forms an opaque or milky emulsion. Used with ordinary care it is not poisonous even for dogs, and, to my mind, besides being the cheapest is one of the most useful and pleasant general disinfectants that can be employed. Potassium permanganate is an efficient though not inexpensive agent for swilling yards and flushing drains, etc., in the strength of I to 50 or 100 of water. It is a good deodorant and fairly effectual in the destruction of bacteria, although not so potent as the previously mentioned agents. Sanitas Fluid has an agreeable aromatic odour, is soluble in water, non-toxic, non-corrosive, and largely used in hospitals and by medical officers of health. In commerce the activity of Sanitas compounds is due to the formation of ozone by the slow oxidation of the resin, camphor, and thymol they contain. In 5 to to per cent. solution, Sanitas is recommended as a valuable disinfectant and deodorant for use in dog kennels, and the Sanitas powder and sawdust are used with good effect, sprinkled upon the floor. Chlorinated lime is a most useful agent, and may be used either dry or in I to 5 per cent. solution.

There are many other preparations suitable for the purposes indicated in this paragraph, among the best being Kresophen, Fecto (contains free chlorine), formaldehyde solution, Izal, etc.

How to Disinfect a Kennel Effectually.-The disinfection of a kennel must be systematically carried out as follows if reliable results are desired: First brush down any cobwebs and dried loose whitewash, then thoroughly sweep away any débris and every particle of litter and sawdust, which must be gathered up and burned. All removable parts, such as the bench or dais, drinking troughs, etc., should be scraped and scrubbed. with 
strong disinfecting solution and soap; then, if possible, place in the sun to dry. In the case of wood, especially that which has become soiled by the dog's excreta; the scraping operation is essential, and better results are obtained if the solution is allowed to soak well in, and remain as long as possible, in direct contact with any organisms which may be present. The walls and floor should next receive attention, both being scraped with a wire brush, then scrubbed down with germicidal fluids; at this stage the kennel may be fumigated, and whether or not this be done, finish off by freely throwing the disinfectant solution over every inch of surface. Finally; where indicated, lime-washing nnay be proceeded with, and in this connection I must lay stress on the fact that to lime-wash over the old dirt, without any preparation, is worse than useless. Yet how often it is done! Limewash is more efficacious than ordinary whitewash, but both are improved by the addition of about half a pint of pure carbolic acid to the gallon. Unslaked lime may be slaked by the addition of 7 gallons of water per I cwt. ; to make the wash use a 20 per cent. solution.

The blow-lamp was greatly used in the army during the late war, and is very effectual indeed provided that the offending organisms are not protected behind débris, etc., and that the flame is made to cover the zohole of the suspected surface. The flame is so often used in a haphazard way that all parts do. not come under its sterilising influence, in which case of course the method then comes into disrepute, and is wrongfully blamed.

(b) Disinfectant Baths for Dogs. - In cases where it is desired to disinfect the body or coat of a dog after recovery from a contagious disease, or after suspected contamination with an infected dog, one must be guarded in the choice of chemicals added to the bath water, since these small animals are very susceptible to poisoning; and-even when insufficient time is allowedfor absorption by the skin, dogs will frequently: lick themselves, with occasionally dire results. : Irritants; 
too, must be avoided on account of the danger to the eyes and to the thin-skinned, vulnerable parts, like the abdomen and scrotum; thus to be on the safe side we must rule out corrosive sublimate, carbolic acid, and Lysol. The recommended medicaments are: Sanitas, 5 per cent. solution; Jeyes' Fluid, 5 to ro per cent. solution; Wright's Coal Tar ; and potassa sulphurata, about I ounce to the gallon.

(c) IRRIgation of Wounds.-The wounds encountered in distemper are confined to the small subcutaneous abscesses, which only occasionally make their appearance, and to the lesions on the abdomen and thighs, produced by the rare exanthematous eruption. In the latter case, if much pus exudes and tends to dry into scabs around the pustules, an antiseptic solution will be requisitioned to wash the parts clean, and for this we may use hydrogen peroxide, 5 to ro per cent. solution in cold water; permanganate of potash, I per cent. solution; Sanitas, 5 per cent. solution; or the parts may be painted with tincture of iodine. An antiseptic much employed during the war, both in human and animal practice, was Eusol, made by dissolving I 2 grains each of good bleaching powder and boric acid in a litre of water, shaking frequently for two hours, and filtering. As a dry application, boracic acid r, Sanitas powder I, and Kaolin Io, is safe and effectual. For washing out abscess cavities, any of the liquids above mentioned may be tried, or preference given to Jeyes' Fluid, 5 to Io per cent., or Kresophen, 5 per cent.

(d) Antrseptic Eye and Mouth Washes.-No irritant application can be tolerated by that very sensitive organ, the eye; thus the universally adopted agent is a warm 3 to 5 per cent. solution of boric acid, or $\frac{1}{2}$ to I per cent. warm solution of potassium permanganate. As a mouthwash either will serve, or perhaps more efficacious would be a 5 per cent. solution of peroxide of hydrogen. Ulcerous spots in the mouth may be dressed with glycerine of tannin. 


\section{BIBLIOGRAPHY}

Hutyra and Marex: Special Pathology and Therapeutics. Müller and Glass: Diseases of the Dog.

WALlis HOARE: System of Veterinary Medicine.

Friedberger and Frohner: Veterinary Pathology.

Finlay Dunn: Veterinary Medicines.

Banham: Posological Tables.

Woodroffe Hill: The Dog and its Management.

Williams: Principles and Practice of Veterinary Medicine. Miller : Practice of Surgery.

Blaine: Canine Pathology.

YouatT: The Dog.

Fleming: History of Animal Plagues.

Veterinary Record.

Veterinary Journal.

Journal of Pathology and Bacteriology.

Bulletin Soc. de Méd. Vét.

Journal of Medical Research.

Recueil de Méd. Vét.

Proceedings of the National Veterinary Medical Association.

Proceedings of the Central Veterinary Society. 


\section{INDEX}

AGE at which dogs attacked, I I influence of, on susceptibility, Io to vaccinate, 60

Alimentary disorders, I 88 lesions, $\mathrm{I}_{42}$

Amaurosis, 97, 203

Anal glands, suppurating, I26 lesions, I 43

Analogous diseases in human beings, 5

Analogy to swine fever, 23

Anasarca, I29

Animals susceptible, 9

Antiseptic eye and mouth washes; 22 I

Arguments for and against Copeman's vaccine, 63

Atrophy of muscles, II6

Ferry's organism, 2I

Bacillus bronchisepticus, arguments for and against, $2 \mathbf{I}$ description of, 34

Bacterial theory of etiology, I5

Bacterins, 7o

Bacteriological notes, 29

Balanitis, 127

Baths, disinfectant, 220

Beef-tea, preparation of, 168

Bibliography, 222

Bile ducts, lesions in, $\mathrm{r}_{44}$

Bitches' milk, infection in, 56

Bladder, distension of, 129,204 inflammation of, 129, 204

Blenorrhcea, 178

Blood, characters post mortem, I47

Blow-lamp as disinfectant, 220

Boarding homes for dogs, 53

Body cleanliness, I62

Brain lesions, I46

Bronchial lesions, I42

Bronchitis, $87, \mathrm{r} 82$

Broncho-pneumonia, 87, I40, I85

Brood bitches, risks attending, 49

Buccal lesions, I45

Carbolic acid, disinfection with, 218

Carcase, characters of, post-mortem 148

Cardiac lesions, 146

Carriers, 28

Cataract, 96, r8o

black, 97, 203

Cats, susceptibility of, 9

Causes, predisposing, 42 theories of, 14
Central nervous system, lesions in, I 46

Cerebral meningitis and sequelæ, III

Chauveau's vaccine, 77

Chemicals in disinfection, 218

Chlorine gas, disinfection with, 217

Chorea, III, I 88

Cleansing the body, I62, 220

the mouth and teeth, 163

Clonic spasms, I08

Clothing, I6o, 217

Clysters, how to give, I7o

Coma, Io7

Communicability to man, 5

Complications of distemper, 85

Conjunctivitis, 92, I77

Constipation, IoI, 195

Contagion, mediate, 47 minimising spread of, $I 6_{4}$

Contaminated articles, disposal of, 46

Convalescence, 2 I2

Convulsions, treatment of, I97

Copeman's bacillus, Dr. Monckton, 24,31

vaccine, 63

Corneal ulceration, 93, I79

Course, 133

Creolin, disinfection with, 219

Croupous pneumonia, 88,185

Curtailing a general outbreak, $5 \mathrm{I}$

Cutaneous lesions, II7, I20, 203

Cystitis, 129, 204

Defective heart, 203

Definition, I, 7

Descemet's membrarie, hernia of, I79

Detecting distemper, 55 , I $5^{\circ}$

Diagnostic value of exanthematous symptoms, II9

Diarrhœa, I00, Igo foods for, 168

Diet, sick, 167

Dietetic treatment, I65

Differential diagnosis, $15^{\circ}$

Discharges, removal of, $16 \mathrm{I}$

Disinfection, 56, 215

by baking and boiling, 2 I 7

by chemicals, 218

by fumigation, 216

by spraying, 217

of a dog by bathing, 220

of a kennel, 219

of wounds, 221

of yards or floors, 218 
Disposal of contaminated articles, 46

Distemper as an entity, 7 classification of, 83

diseases confounded with, I5I

history of, 2

putrid type of, ro3

sequela of, 85 .

signs of previous attack of, $\mathrm{Ir}_{3}$

uncomplicated, 83,92

Distension of the bladder, I29

Dog bureaux, infection in, 52

homes, infection in, 53

shows, spread of infection at, 47 veterinary inspection at, 47

Dogs on approval, risks attending, 50

Dosage of Ferry's vaccine, 7 I

Duration of infectivity after convalescence, 46

Dysentery, I93

Early signs of distemper, 55, r50 stages, temperature in, 85

Emaciation, 103, I23

Emesis, 99, I68, I 88

Enemas, nutrient, I 72

Enteritis, Igo

Entity, distemper as an, 7

Epilepsy, 108, 153, 197 versus rabies, 109

Essentials of isolation, $5^{\mathrm{r}}$

Etiology, bacterial theory of, I5 exotoxin, theory of, 18 ultra-visible virus, theory of, 14

Examination of urine, I28

Exanthematous symptoms, II7 lesions, characters of, 120 diagnostic value of, IIg predilection, seats of, I20

Exercise, 163 treatment of, 22I

Exhibitors' legal liability, 48

Exotoxin theory of etiology, I 8

Extermination of rats, 214

Eye, excision of, 98

lesions, 148

symptoms, 92

treatment of, I77

Fæces, infective nature of, 55, I00

Febrile symptoms, I2I, I75

Feeding per rectum, I 70

Ferry's organism, 34

arguments against, 2 I

vaccine, 68

dose of, 7 I

efficacy of, or otherwise, 73

Filtration experiments with $B$. bronchisepticus, I9

Fits, 108, 197 warning of, I Io
Fomentations, hot, I69

Foods for constipation, 196

for diarrhcea, I68, I93

preparation of, 167

retention of, I68

Foreign nomenclature, I

Formalin, disinfection with, 216

Fumigation, 2I6

Gangrene of the lungs, 90, 188

Gastric lesions, I43

Gastritis, I88

Gastro-enteritis', 98, Ior, 190

General outbreak, curtailing $a_{1} \cdot 5 \mathrm{I}$

Glandular lesions, abdominal lymphatic, 143

bronchial, I42 salivary, ${ }_{4} 6$.

Glaucoma, 97

Gums, lesions on, I45

Hæmaturia, I28, 206

Hæmorrhage of the bowel, 192

Heart, defective, 203

Hemiplegia, Ir6, 201

Hepatic lesions, I.44 symptoms, 104 treatment, 196

History of distemper, 2 sheets for infirmary use, I73

Human beings, analogous diseases of, 5 communicability to, 5

Hydrocephalus, I32

Hygienic treatment, ${ }_{5} 8$

Icterus, 104, 144, 196

Immunity, 58 Wright's theory of, 59

Incontinence, treatment of, 202; 206

Incubation period, 56

Infection, duration of, after convalescence, 46

latent, 28

methods of, 26

sources of, and practical avoidance, 45

Infective matter and its source, 55 nature of fæces, 55, 100

Influence of age and breed on susceptibility, Io

Inhalations, medicated, I6r

Inoculation as a preventive, $5^{8}$

Intestinal lesions, $\mathrm{I}_{42}$ ulceration, I92

Iritis, 95

Irrigation of abscess cavities, 22 I

Isolation, essentials of, 5 I

Jaundice, I04, I44, I96 
Kennel Club, a suggestion"to the, 49

Kennels, well-kept, 54 ! disinfection of, 2 Ig

Keratitis, 97, I78

Kidney lesions, I4G symptoms, I27

treatment, 204

Laryngeal lesions, I42

Laryngitis, 86, I $8 \mathrm{I}$

Latent infection, 28

Legal liability of exhibitors, 48

Leucoma, 96, i8o

Lignière's organism, 32 polyvalent vaccine, $8 \mathrm{I}$

Litter for sick dogs, I59

Lobular pneumonia, 140

Lung lesions, I $^{\circ}$ proportionate frequency of, in distemper, $9 \mathrm{r}$

Lungs, gangrene of, 90 , I88 inflammation of, $87, \mathrm{x}_{4} \mathrm{O}, \mathrm{I} 85$ œdema of, $90, \mathrm{I} 4 \mathrm{I}, \mathrm{I} 88$

Jymphatic glands, abdominal, I43 bronchial, I42

Mange mistaken for distemper, $\mathrm{I}_{54}$ Measles, analogy of distemper to, 5

Mediate contagion, 47

Medicinal treatment, I 73

Meningitis, cerebral, Ir I spinal, Ir7

Methods of infection, 26

Milk of bitches, infectivity of, 56

Miscellaneous symptoms, 123, 203

Mistaken diagnosis, dangers of, I 57

Morbid anatomy, I 40

Mortality, 138

Muscular atrophy, r.r6

lesions, 146

symptoms, 123

Nasal catarrh, 86, I80 lesions, 142

Nephritis, symptoms of, 127 treatment of, 204

Nervous symptoms, Io6 treatment of, 197

Nuclein, I87

Nursing, importance of, 158

Nutrient enemas, composition of, 172 suppositories, $\mathrm{I} 73$

Nystagmus, 98

Ocular lesions, $\mathrm{r}_{4} 8,177$

Edema of the lungs, 90, I4 I, I88

Opacities of the cornea, 95, I80

Opisthotonos, II 6

Oral lesions, 99, I45, I88

Otitis, 126, 206

Otorrhœa, 126, 206
Panophthalmitis, 94

Paralyses, I14, 201 sequelæ of, II6

Paraplegia, II6, 201

Parenchymatous keratitis, 95, I78

Paresis. II 4, 20I

Pasteurella canis, 32

Perchloride of mercury, disinfection with, 218

Period of incubation, 56

Peritoneal lesions, 144

Peritonitis, 124, 194

Permanganate of potash, disinfection with, 219

Pharyngitis, 99, 188

Phisalix vaccine, 78 committee's report on, 80 evidence for or against, 79 organism, 32

Pleural lesions, I4I

Pleurisy, symptoms of, 90 treatment of, 187

Pneumonia, 87 , I40, I85

Post-mortem lesions, 140 typical picture at, $\mathrm{r}_{4} 8$

Predisposing causes, 42

Predominance, seasonal, 8

Premonitory symptoms of distemper 55,84

Prescription, No. I, I77

No. $2,18 \mathrm{I}$

No. $3,18 \mathrm{I}$

No. $4,18 I$

No. 5,182

No. $6, \mathrm{I} 83$

No. 7,184

No. 8,189

No. 9,189

No, I0, I9?

No. II, IgI

No. I2, I9I

No. 13, I9I

No. I4, 204

No. 15,204

Preventive inoculation, 58 with attenuated living cultures, 77

with crude nasaldischarge, 62 with dead cultures, 63

with pure cultures of bacilli, 62

with vaccine lymph, 62

Previous attack of distemper, signs of $\mathrm{a}, \mathrm{II}_{3}$

Priessnitz compress, 186

Prognosis, I 34

Prolapse of rectum, I02, I 43

Public highways as source of infection, 50

Putrid type of distemper, I03 
Quack remedies, 156

Quarantine kennels, distemper in, 54

Rabies, I53 versus epilepsy. 109

Raw meat juice, preparation of, 167

Reactions during vaccination, 6I to Ferry's bacterin, 72

Rectal feeding, I 70 lavage, I 70

Rectum, prolapse of, Io2, I43

Renal lesions, I 46

Report of Committee on Phisalix Vaccine, 8o

Resistance of contagium to cold or heat, 56

Respiratory'symptoms, 86 , I8o lesions, 140

Retention of food, aids for the, 168 of urine, 129

Rhinitis, 86 , I80

Risks attending brood bitches, 49 dogs " on approval," 50

Room temperature, 159

Salivary glandular lesions, I4 6

Seasonal predominance of distemper, 8

Secondary invading organisms, $3^{8}$

Sequelæ of cerebral meningitis, I I I of distemper, 85 of paralysis, II6

Sero-therapy, 209

Sick diet, 167

Signs of a previous attack of distemper, II3

Sources of infection and their practical avoidance, 45

Spinal cord, lesions of the, I47 meningitis, II7

Splenic lesions, I 48

Spraying disinfectants, 2 I 7

St. Vitus' dance, I I I, I98

Staphyloma, 94, 180

Steaming the head, $16 \mathrm{I}$

Stuttgart disease, 152

Subnormal temperatures, I 23

Sulphurous acid gas, disinfection with, 216

Suggestion to the Kennel Club, 49

Suppurations, I25, I4I, 209 of anal glands, 126

Surgical conditions, $\mathrm{I}_{43}$

Susceptibillity, influence of age and breed ơr r ro
Susceptible animals, 9

Swine fever, analogy of, to distemper,

23

Symptoms, 82

complicating, 85

exan thematous, II7

diagnostic value of, I I9

eye, 92

febrile, I2I

gastro-intestinal, 98

hepatic, $\mathrm{IO}_{4}$

miscellaneous, I23

muscular, I23

nervous, 106

premonitory, 55, 84

respiratory, 86

Synonyms, 2

Temperature charts, I2I, I22

in the early stages, 86

room, I59

subnormal, I 23

Tenesmus, IO2

Thermometer, transmission of infection by, 56

Tonsillar lesions, I45

Tracheal lesions, 142

Treatment, ${ }_{5} 6$

dietetic, 165

empirical, 156

hygienic, 158

medicinal, 173

Tuberculosis, 152

Ulcerated corriea, 93, I79 bowels. I92

Ultra-visible virus theory of etiology, 14

Uncomplicated distemper, 85,92

Urine, examination of, 128

Urino-genital organs, 206

Vaccination, 58 age for, 60 reactions during, $6 \mathrm{I}$

Vaccine, Chauveau's, 77 Copeman's, 63

Ferry's, 68

Lignière's, 8I

Phisalix, $7^{8}$

Veterinary inspection at dog shows, 47

Vomiting, 99, I68, I 88

Well-managed kennels, 54

Whey, how to prepare, 167

Wounds, disinfection of, 22I

Wright's theory of immunity, 59 






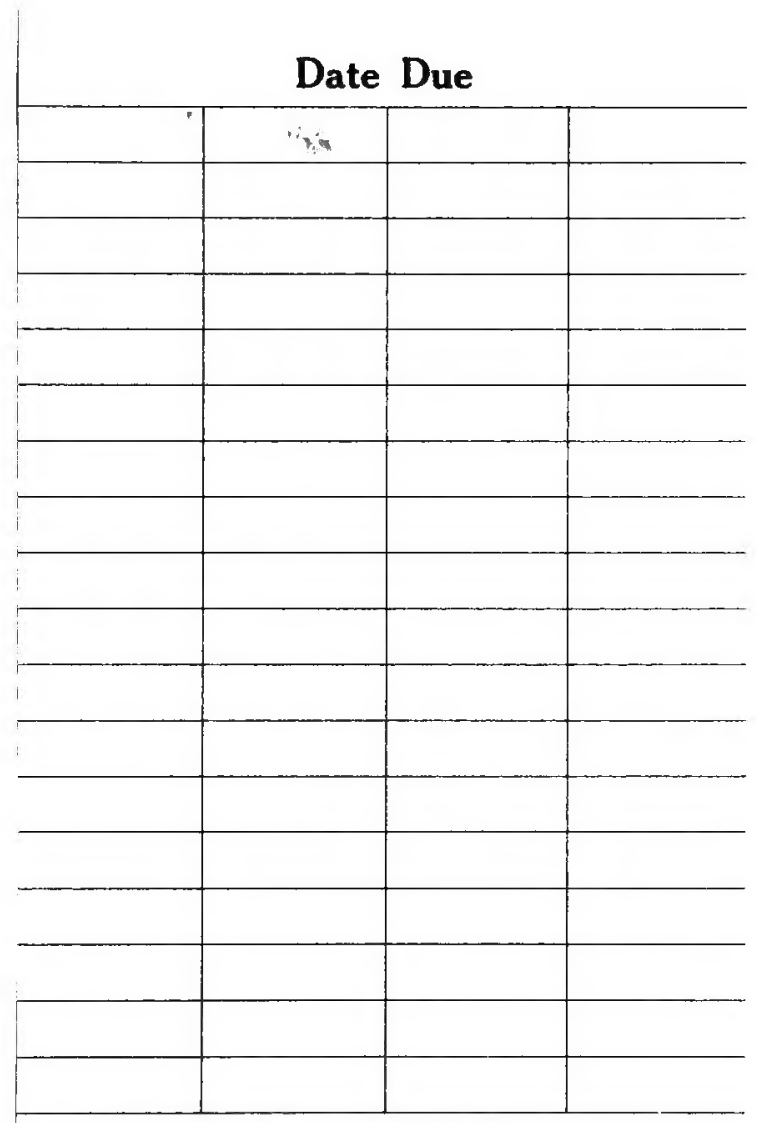

Library Bureau Cat. No. 1137 
